
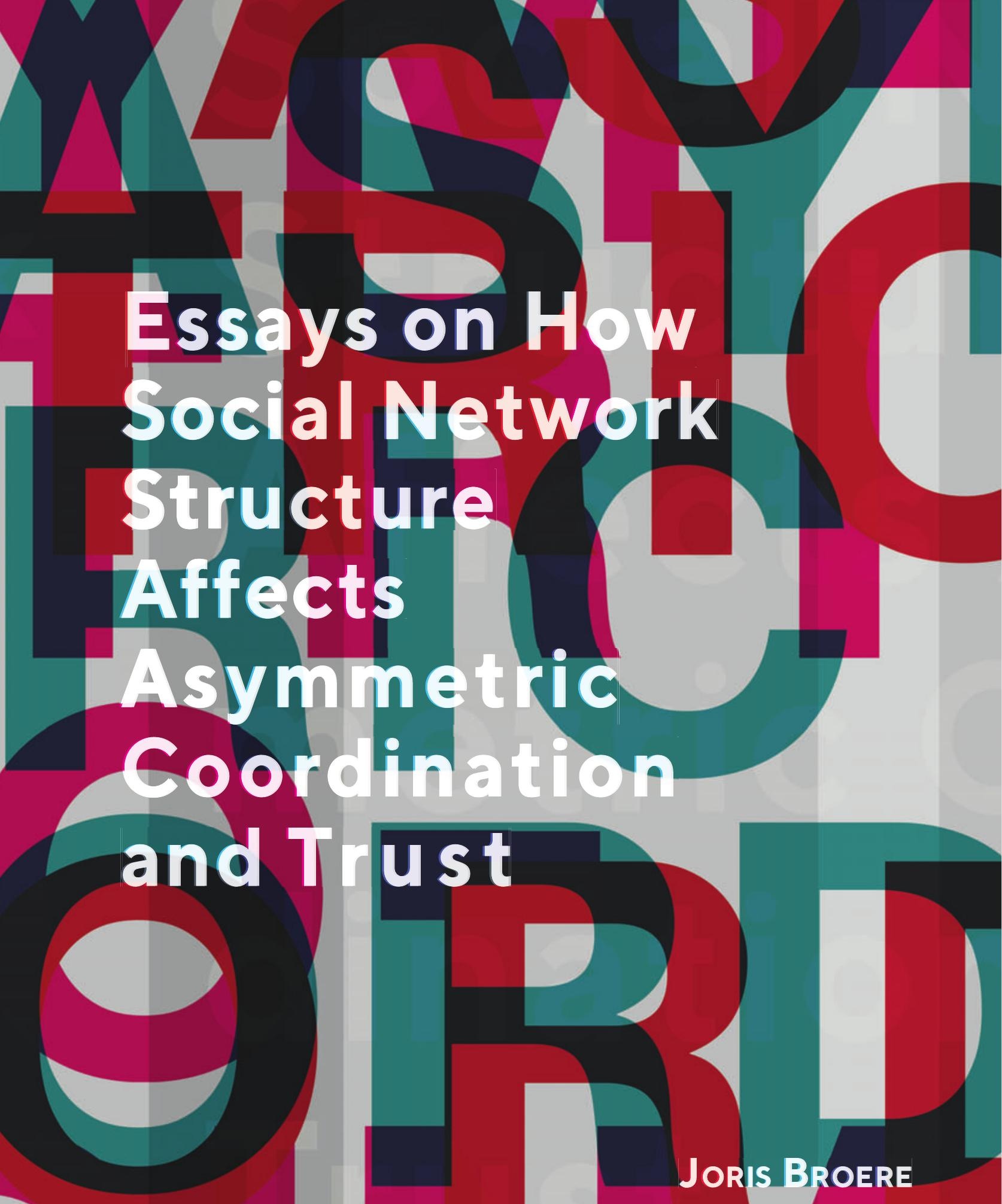

Essays on How Social Network Structure Affects Asymmetric Goordination and T ust

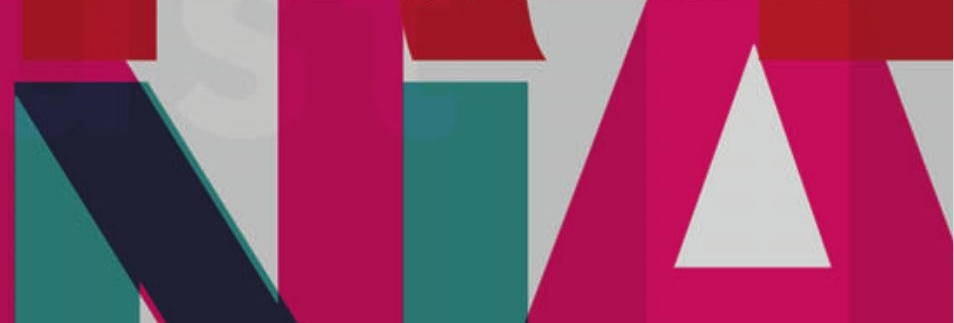



Essays on how social network structure affects asymmetric coordination and trust

Joris Jan Broere 


\section{Manuscript committee:}

Prof. dr. ir. H.A. Dijkstra (Utrecht University)

Prof. dr. A. Flache (University of Groningen)

Prof. dr. P.G.M. van der Heijden (Utrecht University)

Dr. P. Hernández (University of Valencia)

Prof. dr. A. van de Rijt (European University Institute)

Essays on how social network structure affects asymmetric coordination and trust - Joris Jan Broere

Cover design: Giacomo Gallo

Printing: Ridderprint BV | www.ridderprint.nl ISBN: 978-94-6416-296-7

(C) Copyright Joris Jan Broere, 2020

All rights reserved. No part of this publication may be copied, reproduced or transmitted in any form of by any means, electronic or mechanical, including photocopy, recording, or any information storage or retrieval system, without the prior written permission of the author. The copyright of the published articles has been transferred to the respective journals. 


\title{
Essays on how social network structure affects asymmetric coordination and trust
}

\author{
Essays over hoe de structuur van sociale netwerken
} asymmetrische coördinatie en vertrouwen beïnvloedt

(met een samenvatting in het Nederlands)

\begin{abstract}
Proefschrift
ter verkrijging van de graad van doctor aan de

Universiteit Utrecht

op gezag van de

rector magnificus, prof.dr. H.R.B.M. Kummeling, ingevolge het besluit van het college voor promoties in het openbaar te verdedigen op
\end{abstract}

vrijdag 20 november 2020 des middags te 4.15 uur

door

Joris Jan Broere

geboren op 6 februari 1987

te Amersfoort 
Promotor: $\quad$ Prof. dr. ir. Vincent Buskens

Promotor: Prof. dr. ir. Hendricus Theodorus Christiaan Stoof

Dit proefschrift werd mogelijk gemaakt met financiële steun van het Complex Systems Fonds, met dank aan Peter Koeze. 


\section{Contents}

List of Tables viii

List of Figures $\quad$ x

1 Synthesis 1

1.1 Complex Systems Science . . . . . . . . . . . . . . . . 6

1.2 Complex Systems Science and Sociology . . . . . . . . . . . . . 10

1.3 Part 1: Chapters 2 till 4 . . . . . . . . . . . . . . 14

1.4 Part 2: Chapter 5 . . . . . . . . . . . . . . . 18

1.5 Future research . . . . . . . . . . . . . . . . 20

Part I Cohesion and polarization in asymmetric coordination

2 Network effects on coordination in asymmetric games 25

2.1 Introduction . . . . . . . . . . . . . . . . . . . 26

2.2 Methods . . . . . . . . . . . . . . . . . . . 29

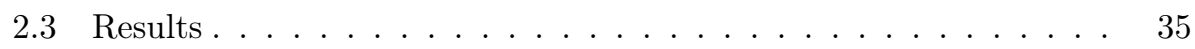

2.4 Discussion . . . . . . . . . . . . . . . . . . 40

3 An experimental study of network effects on coordination in asymmetric games $\quad 43$

3.1 Introduction . . . . . . . . . . . . . . . . . . . . . . 44

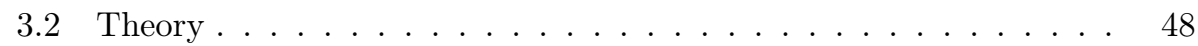

3.3 Methods . . . . . . . . . . . . . . . . . 51

3.4 Conclusion and discussion . . . . . . . . . . . . . . 58

4 Asymmetric games on networks and the Ising model 61

4.1 Introduction . . . . . . . . . . . . . . . . . . . . . . . 62

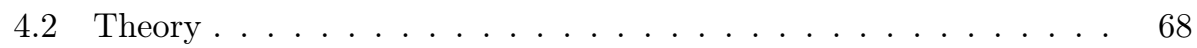

4.3 Methods .......................... 71

4.4 Results......................... . . . . 72

4.5 Conclusions and discussion . . . . . . . . . . . . . 76

Part II Cohesion in dependency and complementary

5 The division of labor and generalized trust:

$\begin{array}{ll}\text { Organic solidarity revisited } & \mathbf{8 1}\end{array}$

5.1 Introduction . . . . . . . . . . . . . . . . . . . . 82

5.2 Theory. . . . . . . . . . . . . . . . . . . . . . 88

5.3 Data and methods . . . . . . . . . . . . . . . . . . . . . . . . . . . . .

5.4 Results . . . . . . . . . . . . . . . . . . . . . 96

5.5 Conclusion and discussion . . . . . . . . . . . . . . 97

$\begin{array}{ll}\text { Appendices } & 101\end{array}$ 
Nederlandse Samenvatting

Bibliography

Dankwoord (Acknowledgements)

Publications and working papers by the author 




\section{List of Tables}

1.1 Cooperation problem represented as a prisoners dilemma. . . . . . 14

1.2 Payoff table of an asymmetric coordination game . . . . . . . . . 15

1.3 Example payoff table 3 player asymmetric 'battle of the sexes' game. 16

2.1 Payoff table of an asymmetric coordination game . . . . . . . . . 26

2.2 Possible payoff situations in BoS on a network, where $0<S<1 \quad$. 29

2.3 Number of neighboring nodes required for a local majority . . . . . 33

2.4 Descriptives of the dependent and independent variables. . . . . . 35

2.5 Regression results, standardized, dependent variable Power. . . . . 39

3.1 Example payoff table asymmetric 'battle of the sexes' game . . . . 45

3.2 Example payoff table 3 player asymmetric 'battle of the sexes' game. 46

3.3 Number of neighboring nodes required for a local majority . . . . . 50

3.4 Payoff table for the experiment . . . . . . . . . . . . . . . . 52

3.5 Correlation between the computational model and the empirical results. . . . . . . . . . . . . . . . . . 55

3.6 Multilevel logistic regression results . . . . . . . . . . . . . . 57

4.1 Payoff table asymmetric 'battle of the sexes' game and an energy landscape that models the same dilemma as the battle of the sexes game. ....................... 65

5.1 Logistic regression with dependent variable generalized trust, $\mathrm{N}=2867$. 96

A1 Regression results with deterministic myopic best response . . . . . 103

A2 Regression results with $\mathrm{N}=40$. . . . . . . . . . . . . . 111

A3 Regression results with $\mathrm{N}=80$. . . . . . . . . . . . . . . 111

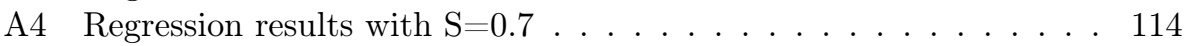

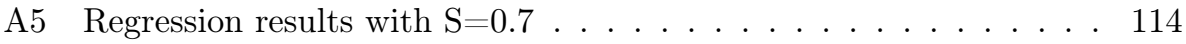

A6 Adjacency matrix of the random network . . . . . . . . . . . . 116

A7 Adjacency matrix of the clustered network . . . . . . . . . . . . . 117

A8 Adjacency matrix of the centralized network . . . . . . . . . . . . 118

A9 Logistic regression results with method by of Tacchella et al., (2012) 124

A10 Logistic regression results for the cutoff value $\mathrm{RCA}=1.5$. . . . . 126

A11 Tabel voor een asymmetrisch coördinatie spel . . . . . . . . . . . . 129

A12 Tabel voor een asymmetrisch coördinatie spel met drie speleres . . 130 



\section{List of Figures}

1.1 Fields arranged by purity . . . . . . . . . . . . . . . 11

1.2 Coleman boat . . . . . . . . . . . . . . . . . 12

2.1 Proportion of $\alpha$ played in a network after convergence . . . . . . . 36

2.2 Kernel regression plot . . . . . . . . . . . . . . . . . . 37

2.3 Density of node Power . . . . . . . . . . . . . . . . . . . . 38

3.1 Four-player games, represented as a network; $\alpha$ and $\beta$ denote the preferences of the players. . . . . . . . . . . . . . . . 47

3.2 20-player games, represented as a network. . . . . . . . . . 53

4.1 Networks where the colours indicate the preferences . . . . . . . 72

4.2 Average magnetization $m$. . . . . . . . . . . . . . . 73

4.3 Probability of choosing $\alpha$. . . . . . . . . . . . . . . 75

A1 Variance of the proportion of $\alpha$ played on a network when the same initial distribution . . . . . . . . . . . . . . 106

A2 Network and the estimate of node Power . . . . . . . . . . . . . . 107

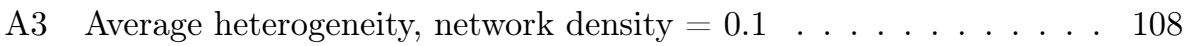

A4 Average heterogeneity, network density $=0.2 \ldots \ldots$. . . . . . 109

A5 Average heterogeneity, network density $=0.3 \ldots \ldots . . . . . \quad 110$

A6 Proportion of $\alpha$ played in a network after convergence for $\mathrm{S}=0.7 \quad$. 112

A7 Proportion of $\alpha$ played in a network after convergence for $S=0.7$. 113

A8 Instructions of the experiment in English . . . . . . . . . . . . 119

A9 Instructions of the experiment in Spanish . . . . . . . . . . . . . 120

A10 Example first round computational and experimental results random network . . . . . . . . . . . . . . . . . . 121

A11 Example last round computational and experimental results random network . . . . . . . . . . . . . . . . . . 121

A12 Example first round computational and experimental results small world network . . . . . . . . . . . . . . . . . . . . . . 122

A13 Example last round computational and experimental results small world network . . . . . . . . . . . . . . . . .

A14 Example first round computational and experimental results Network with central node network . . . . . . . . . . . . . . 123

A15 Example last round computational and experimental results Network with central node network . . . . . . . . . . . . . 123

A16 City-occupation matrix . . . . . . . . . . . . . . . 125 

Chapter 1

Synthesis 

"Well, I am no rocket scientist, and I have immense respect for people who can land a machine the size of a car on another planet. But the sad fact is that we're actually much better at planning a flight path of an interplanetary rocket than we are managing the economy, merging two corporations, or even predicting how many copies a book will sell. So why is that rocket science seems hard, whereas problems having to do with people - which arguably are much harder - seem like common sense?"

- Duncan J. Watts (Watts, 2011)

The substantial topics studied in this dissertation are coordination problems in social dilemma situations and how general trust is established. The paragraphs below are stylized examples of the studies' mechanisms. Example 1a till Example $1 \mathrm{~d}$ are to illustrate how a coordination problem can be a social dilemma. Example 2 illustrates how general trust mechanisms can be promoted in social structures.

Example 1a: Imagine, you and a colleague are working on a project together. You remember you two had a dispute on which text program you wanted to use. You want to use the program 'Word' and your colleague wants to use the program ' $\mathrm{LT}_{\mathrm{E} X} \mathrm{X}$ '. At the time of the dispute, it was already hard to decide which program to use, because neither of you had a clear argument on why the preferred program was better. However, now you cannot recall which program you ended up choosing and you cannot contact your colleague in order to ask. If you don't choose the same program to work with as your colleague, the work is for nothing and you probably don't make the deadline. Which program would you choose to use?

Example 1b: You are in the same situation only with three colleagues. Again, no clear decision has been made regarding which program to use. Let's assume you know the two other colleagues also don't know which program to use and you are again unable to contact each other. You still have your preference for Word, however, your two colleagues both have a preference for $\mathrm{IAT}_{\mathrm{E} X}$. What would you choose in this situation? Is your reasoning and/or decision different from a week before?

Example 1c: A week later, you and your colleagues haven't learned from your past mistakes. Again, nobody wrote down the minutes when the decision was 
made on which program to use. You are working with the same two colleagues as the week before. You still have your preference for the program Word and your two colleagues still prefer $\mathrm{L}_{\mathrm{E}} \mathrm{X}$. Again, you do not know which program you ended up choosing to work with. Again, choosing the wrong one means you don't make the deadline. However, you do know that last week both your colleagues choose to work in $\mathrm{IT}_{\mathrm{E}} \mathrm{X}$. What would you choose in this situation? Is your reasoning and/or decision different from a week before?

Example 1d: A huge project is coming up. It is a twenty person project and again no clear decision has been made on which program to use. You ask your two colleagues what they prefer. One prefers Word, the other $\mathrm{IAT}_{\mathrm{E}} \mathrm{X}$. Since you also prefer Word, the three of you decide to go with Word. However, one hour later the colleague who prefers $\mathrm{A}_{\mathrm{A}} \mathrm{E} \mathrm{X}$ talked to a fourth colleague who also prefers ${ }_{\mathrm{A}}^{\mathrm{A}} \mathrm{T}_{\mathrm{E}} \mathrm{X}$. The colleague tells you she changed her mind to $\mathrm{L}_{\mathrm{E}} \mathrm{X}$ as well. What will you do now? Also change to $\mathrm{H}_{\mathrm{T}} \mathrm{T} \mathrm{X}$ or stick with Word?

Example 2: A big project came in. It's a prestigious case and you'll receive a large bonus when you finish it in time. However, the deadline is very soon. You figure you can make it, but the work has to be done very efficiently. Your boss also understands this and proposes that you can choose the people you want to work with. The first option is to work with two colleagues with whom you are sharing an office. You know them and get along well. You have similar skills, but you figure that if (and only if) you manage to work as a team, you'll get the work done just before the deadline. However, free-riding behavior would be detrimental to the project. The second option is you work with two people from other departments. You have never met these two people, but both have a very different skill set compared to you. You figure the project can be divided in three parts, and each part can be perfectly matched to the skill set of the people in your team. The project can be done very efficiently and it takes less time than with your close colleagues, but you figure you'll make the deadline if (and only if) all team members do their part. In both options the obtained bonus will be equally split over the team members. Which option would you choose? Why?

Example 1a until Example 1d are stylized situations on how people choose to coordinate when they prefer different options. In these examples there is a tension between what people prefer and the need to choose the same as others. Example 2 is on how trust is established in groups of people. The question in Example 2 is whether you rather work with people you know or with people who have complementary skills to you. If you choose for the first option, you probably 
figured that you know those colleagues well and you get along. The knowledge on past interactions with them will create the trust needed to work as a team and finish the project. If you choose for the second option, you probably figure that a complementary skilled team can do the job efficiently and the people in this team will do their work because not doing it means they don't get the bonus. You even might have figured that while you don't know them, they belong to the same company, and the institutional context might provide some extra security. In the first case, familiarity and personal relations will create the basis for trust. In the second case, complementary and institutional context.

What the above examples have in common is that they both relate to the sociological problem of social cohesion. In the first part of this dissertation, we study a particular kind of cohesion problem where people want to cooperate/coordinate, but differ in their opinions on how to achieve the goal. In this situation there is a tension both for the individual and in the group as a whole. On the group level, cooperation means that some people may not get their preferred option. For the individual the tension is between behaving cooperatively and trying to get what you want with the risk that there will be no cooperation at all. In this dissertation, we hypothesize that the social interaction structure (or network structure) might help and shape this process. Some networks might facilitate unity, while other network structures might have a polarizing effect. In Chapters 2, 3 and 4 the effect of network structure on coordination problems with asymmetric preferences is studied by means of different methods.

In the second part of this dissertation, we study the influence of social interaction on cohesion on a more abstract level. Social interaction is not only shaped by individuals who choose to interact with others. People interact, cooperate and coordinate with others they do not know on a daily basis. Social interactions are also shaped by the way society is organized. How a society is organized is regulated by its institutions. We focus on one such organization (or institution), namely the division of labor. In this second part of the dissertation we test an old hypothesis that economic dependencies that emerge from a division of labor can be a source of social cohesion. In Chapter 5 we aim to empirically measure aspects of the division of labor and relate these measures to indicators of social cohesion.

In order to study these problems, we borrow ideas and methods from the relatively new field of 'complex systems science' and more specifically 'network science'. Because complex systems science has been such an important part in writing this dissertation, I start with a small overview of the main ideas, before introducing 
the individual chapters of this dissertation in more depth. The goal is to give some background knowledge to the main ideas and methods in this dissertation and how they can be useful in the field of sociology. And it is also meant as an small introduction to complex system science for those who do not know complex system science, and its connection to sociology for those who do not know sociology. However, it is not meant as an exhaustive overview of neither scientific fields. After this short introduction, the goals, research questions, and the results of the individual chapters will be discussed in more detail.

\subsection{Complex Systems Science}

Although complexity science is becoming rapidly more popular, an agreed upon exact definition of a 'complex system' is still lacking (Ladyman et al., 2013). A 'complex system' is often described by its characteristics such as many interacting components, non-linear behavior, feedback, sensitive dependence on initial conditions and emergent behavior. However, non of these characteristics can be considered as necessary or sufficient conditions to define a complex system (Ladyman et al., 2013). One reason is that for every of these characteristics there are systems that are considered 'complex' that do not have these requirements, while there are systems that are not considered 'complex' that do have some of these characteristics. Another reason is that important characteristics such as 'emergence' lack an agreed upon definition themselves (Ladyman et al., 2013). One could say that the definition of a complex systems is an interaction of different characteristics, that is sensitive to the object of study, with different emergent properties for different academic disciplines. Because of the lack of definition, it might be more useful to think about when an object of study can benefit from thinking about it as a system that is 'complex'. Therefore, in what follows the concepts are introduced as they are regarded in this dissertation, together with a discussion of the added value of thinking about sociological phenomena in these terms.

In this dissertation, complex systems science is the study of how interacting components bring about patterns or behavior that are characteristic for the total system. The object of study is not so much the components that form the system, but rather the interactions that together describe some properties of the system as a whole. What makes a complex system distinct form a 'regular' system is that the system as a whole is more effectively described by system-level properties compared to describing the system by the properties of the components that form the system. So, complex system science studies how components in a system 
interact with each other bringing about global or 'macroscopic' properties of a system. As most things in nature or science do not just appear on their own and are completely self sufficient, many studied subjects in the full range of science can be considered either a component of a complex system or a complex system itself. Some examples of complex systems are, a cell, a brain, organisms, the internet, a power grid, markets, a society, an eco-system or the universe.

Take for instance the (human) brain. The brain consists of billions of neurons that interact with each other. However, in studying the brain, it does not make much sense to only describe it as a set of neurons firing. In order to learn something about the brain it is easier to describe it at a higher level, for instance by describing its functionality. Examples of higher level phenomena of the brain could be information processing, coordination of bodily functions or consciousness. It is often hard to think of these phenomena in terms of exact configurations of neurons firing. With a phenomenon like consciousness it is even hard to imagine how this emerges from neural activity at all. This is because higher level phenomena, like consciousness, have properties that the lower level neurons seemingly do not have. Yet, neural activity is most likely responsible for all of these phenomena. When these higher level phenomena are best described by properties that are usually not the properties that best describe the lower level interactions, this is referred to as 'emergence' or 'emergent phenomena'. Emergence is one of the key concepts in complex systems science and throughout this dissertation.

\subsubsection{Emergence}

One reason to study a system of components is when the behavior of the system has emergent phenomena. Emergence occurs when all components together can have properties that the individual components do not have themselves. The same holds for sociological phenomena. At the basis of most sociological phenomena are social interactions (or social networks). Social interactions are to a large degree structured. For instance by informal institutions, such as culture, or more formal institutions, such as a legal system. Just like the brain, it does not make sense to study all the individual social interactions. Instead, sociologists try to describe patterns in these human interactions that bring about a phenomenon. How a society functions can be regarded as an emergent phenomenon of how its social interactions are structured, as it does not make sense to describe the collective phenomenon in terms of the individuals in it.

So emergent phenomena have properties that the lower level components do not 
have. But does this mean that emergent phenomena are something more than the patterns and lower level behavior that causes them? In order to answer this question let us see if emergence can be explained in a reductionist view.

\subsubsection{Reductionism}

Complex system science is often contrasted to reductionism. Reductionism is the idea that (complex) phenomenon can be reduced to the (interaction) of their parts or more fundamental parts, where the parts behave in some regular way. The phenomenon to be explained is therefore equal to summing its (more fundamental) parts. So the question is if we can understand emergent phenomena by summing their parts, even though the emergent phenomena have properties that the components do not?

In order to think about the question above, let's do a thought experiment called Laplace's demon. Laplace's demon is the (philosophical) idea, that if someone (the demon) knows the precise location and momentum of every atom in the universe, their past and future values for any given time are entailed; they can be calculated from the laws of classical mechanics. Although in the light of current scientific knowledge on classical mechanics and quantum physics (which I know nothing about), this conception of Laplace's demon is most likely wrong, the idea still serves as a thought experiment.

Could Laplace's demon understand properties of an emergent phenomenon by knowing, comprehending and understanding all micro-level behavior? If Laplace's demon knows the exact position and behavior of all neurons in a brain, could it derive a higher level property like 'consciousness' from this? Or if Laplace's demon knows all social interactions in a group of people, can it derive the culture? In the first example the question is whether 'consciousness' is an ontological difference from the set of neurons or 'just' an epistemological difference. When consciousness is ontological different from the set of neurons, this means that consciousness and a set of neurons are two fundamentally different things or entities. When consciousness and neurons are epistemological different and not ontological different, this means that both are the same thing, it is just too difficult to comprehend or understand consciousness as the set of interacting neurons. In philosophy this is called 'conceptual dualism' (Papineau, 2002; Davidson, 1980). This means that we have different ways of thinking about the same substance. The 'physical event' of neurons firing might be the same substance as 'the experience' of consciousness, but to talk about the physical event and the experience we need a different set of 
words. So while 'consciousness' and 'a set of interacting neurons' are actually the same thing, it is too complicated to express 'the set of interacting neurons' in such a way that it means the same as 'consciousness'. For an emergent phenomenon like culture, it is easier to see how this is just an epistemological difference. Because culture itself is not an entity, just a collective noun for a set of humans and their patterns of interactions. The same holds for ant colonies or bird flocks. One can simulate ant colonies and bird flocks and the result will be exactly equal to the sum of behaviors of the ants and their interactions. However, when we talk about their collective behavior, you don't want to explain each ant and each interaction.

\subsubsection{Reductionism and emergence}

Complexity has been used to describe phenomena where properties at a higher scale cannot be reduced to properties at a lower scale. A popular phrasing is to say that the whole is more than the sum of its parts. Or isn't it? I think this is a common mistake, or it seems to suggest at the very least something that it is not, as the whole is always exactly the sum of its parts. So why is a concept like emergence useful? The problem is that we don't know what to sum to get to the whole or, when we do know the sum, it does not provide useful information. Scientific knowledge is often not to sum, but to summarize. The sum itself often does not provide us the interesting scientific information. So I think most emergent phenomena are epistemological differences between the macroscopic properties and the microscopic behavior that causes it. The confusion arises because there is often a 'conceptual dualism' between the agents and the emergent phenomenon. It is simply too difficult to express the emergent phenomenon in terms of its agents in an effective manner. For instance, when describing a 'car' to someone who has never heard of a car before, it is often not very useful to give a list of the parts it is made of and how they interact. It is more useful to give a summary of its purpose. We understand scientific knowledge as describing something complex or difficult as simple as possible. The art of complexity science is therefore to describe some 'complex' phenomenon, as simple as possible.

Reductionism is compatible with the complex systems perspective, when we accept that agents are not closed systems (Carroll, 2017). Agents have inputs and outputs, and how you structure the in and outputs across agents is what is causing emergence. However, the behavior can still be understood by the behavior of individual agents given some input pattern. So within complex system science, the object of study is not the agent itself, but the pattern it produces when it interacts 
with an environment and/or other agents, changing the subject of study from the agent to feedback patterns between agents or environment. Emergent phenomena can be studied by recurring patterns that occur with events of interest. The first step is recognizing the patterns. The second step is understanding the origin of the regularity (Holland, 2000).

So what do we do when we describe a system in terms of emergent properties? Laplace's demon can predict the next state with exact precision from the previous one by knowing all the parameters in the system and having infinite computational power to predict the next. Emergence is knowing only a small number of parameters in the system, but being able to predict the next state with a reasonable certainty.

\subsection{Complex Systems Science and Sociology}

Sociology could be regarded as a complexity science par excellence. The definition of sociology on Wikipedia reads: 'Sociology is a study of society, patterns of social relationships, social interaction and culture of everyday life' (Wikipedia, 2019). What should be clear from this definition is that sociology consists almost entirely of emergent phenomena, with some key concepts like 'patterns' and 'interaction' already present in its definition. Society could be regarded as a complex adaptive systems and many of its objects of study, such as social cohesion or inequality, are emergent phenomena (Waldrop, 1993; Eve et al., 1997).

That sociology is a complexity science par excellence is exactly at the root of the problem stated in the quote by Duncan Watts at the beginning of the chapter. Let's take one step back to Laplace's demon and assume we know the fundamental building blocks of the universe and how they act according to deterministic laws. Laplace's demon can calculate the past and the present if it knows the precise location and momentum of every atom in the universe. Laplace's demon could also calculate sociological phenomena with 100 percent accuracy. However, in explaining the sociological phenomena, lets say social inequality, Laplace's demon could only inform us on how all the fundamental building blocks in one state got into another according to some law. This would not be very informative to the sociologist, so the sociologist asks the demon to explain the phenomena on a higher level. The demon goes on and explains the sociological phenomena in terms of properties of matter and how they interact. The demon's story loses precision, but the story becomes more tangible to the sociologist. However, the sociologist 


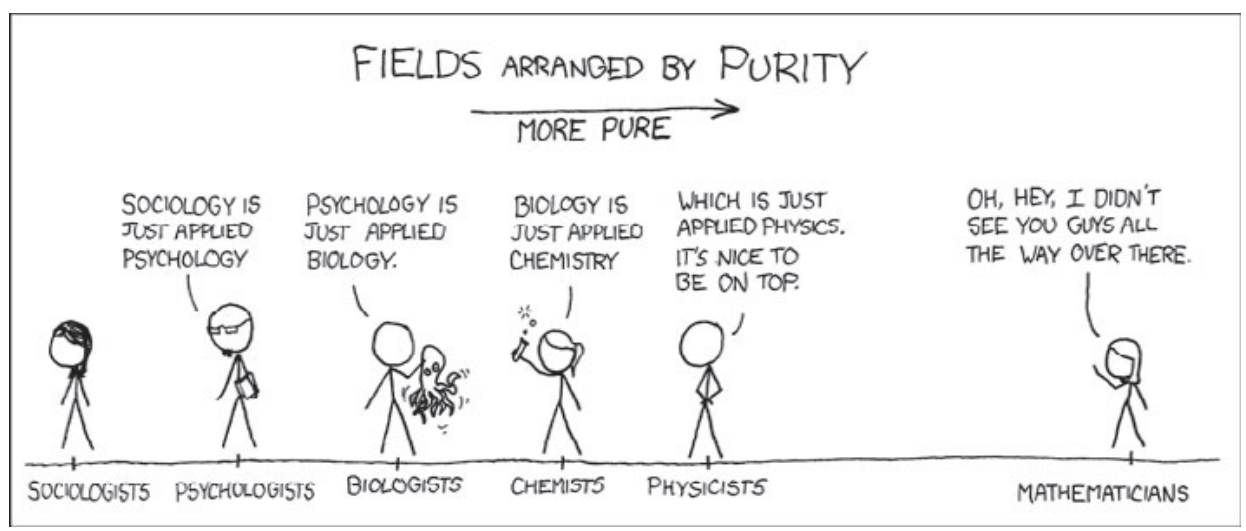

Figure 1.1: When the title would be 'Fields arranged by Complexity' the arrow might point to the other direction $(\mathrm{xkdc}, 2019)$.

is still not satisfied with the demon's explanation and asks again to explain the phenomena on a higher level. The demon tells the story again, however, this time in terms of living organisms, their chemical processes and molecular interactions. The demons story loses again precision, but the sociologist really gets the feeling that he is starting to understand the demon. However, not quite yet, and the sociologist asks again to explain the phenomena on a higher level. The demon goes on and explains of every individual in a society which choices they made that caused the social inequality. The sociologist finally thinks he fully understands and asks the demon, do I summarize it correctly by saying that all individuals maximize utility?

So to answer Duncan Watt's question, sociology could be regarded physics with numerous levels of emergence. At every level of emergence, one loses precision in the predicted event (or as Figure 1.1 puts it, the prediction becomes less pure). For instance, by assuming that people maximize utility, you can probably describe some important patterns in society, but you also miss information, as that human decision making is much more complicated than a simple utility function. A human has a brain, with chemical processes that produces patterns, but also deviations from those patterns. All those factors together make that a society produce many degrees of freedom and high amounts of uncertainty. Thereby, it is not useful to talk about sociological problems in terms of configurations of atoms. However, this does not mean that sociology is a lost cause.

In a sense, sociologists have tried to deal with complexity for decades. For many 


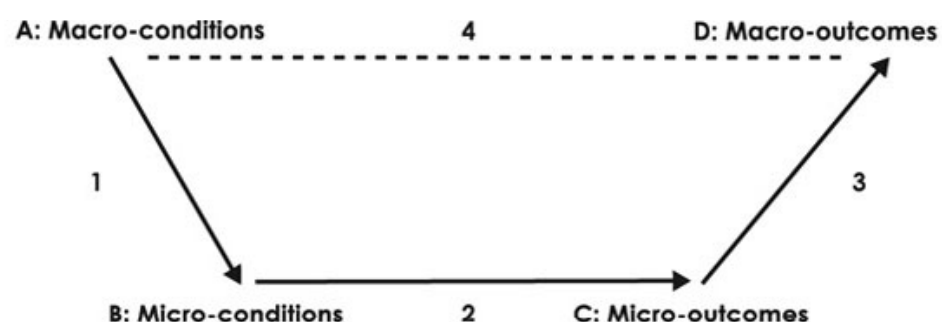

Figure 1.2: Coleman boat, where $\mathrm{A}$ and $\mathrm{D}$ represent propositions describing macroconditions and macro-outcomes, $\mathrm{B}$ and $\mathrm{C}$ represent propositions describing microconditions and micro-outcomes, Arrow 1 represents assumptions on how social conditions affect these variables, Arrow 2 represents the micro-theory, Arrow 3 represents the transformation rule of how individual behavior generates macro-outcomes, Arrow 4 represents an empirical regularity at the macro-level.

sociologists, complexity science, or complexity thinking should sound familiar. For instance, many elements of network science find their roots in sociology (Freeman, 1977). However, the most obvious connection between complex systems science and sociology is the micro-macro link in James Coleman's 'boat' scheme (Coleman, 1994).

The Coleman boat represented in Figure 1.2 is considered to be the blue print of sociological theory building (Raub et al., 2011). Sociological theory is concerned with explaining how certain macro-conditions cause macro-outcomes, represented by node A and D in Figure 1.2. In sociological theory, macro-level phenomena often refer to the social system, such as a society or culture, and the micro-level refers to the level of the individual. Coleman's claim here is that macro conditions and outcomes are mere empirical regularities. For a sociological theory to claim causality, the theory needs to take the behavioral level into account.

Arrow 3 of the Coleman boat in Figure 1.2 represents the transformation rule. The transformation rule describes how individual behavior leads to macro-outcomes. To see why this is relevant, let's take a look at the most recent Presidential elections in the United States of America between Donald Trump and Hillary Clinton. The Coleman boat can be used to explaining why Trump won the election. However, now we only focus on how the individual votes (node $\mathrm{C}$ in Figure 1.2) lead to the macro-outcome of who is elected. If the elective system (transformation rule) would have been majority rule, the individual votes would be summed. The presidential candidate with the most votes would win the election, in case of majority rule, Hillary Clinton. However, the USA has an electoral district system. The 
district system is an indirect election in which an electoral college is chosen to represent a state. The electoral college in its turn chooses the President, in this case Donald Trump. In this example it becomes clear that the same micro-outcomes can result in completely different macro-outcomes by changing the transformation rule.

So when is it useful to think about a sociological problem as a complex system? I think this has all to do with the transformation rule, Arrow 3 in Figure 1.2. When the macro-outcome is an aggregate of the micro-outcomes, the macro-outcome is the same as summing the micro behaviors. In this case there is no interdependence between the actors and complexity science is of not much use. However, when there is a discrepancy between the individual micro-outcome and the observed macrooutcome, it might be useful to think about the empirical deviation as a complex system.

One of the first and most famous examples of modeling the micro-macro link is Schelling's segregation model (Schelling, 1969; Schelling, 1971). Schelling studied residential segregation in the United States of America in the late 1960's. The observation was made that even though the attitudes towards racial residential segregation where changing significantly towards more positive attitudes for more residential integration, the observed residential segregation stayed the same. Schelling developed an agent based model where the decisions of the agent are dependent on the decisions of other agents. In the model, agents have a preference to be around a certain amount of similar others. If this preference is not met they move somewhere else and if this preference is met they stay where they are. The model indicates that when the agents have very mild preferences for being around of similar others, complete residential segregation can emerge. Therefore, the model can explain the emergence of residential segregation even though the individual agents do not want residential segregation to happen. A remark has to made here that a similar model has been published before Schelling published his version (Hegselmann, 2017; Sakoda, 1971). Other examples of modeling the transformation rule as a complex system are Granovetter's threshold model (Granovetter and Soong, 1983). This model explained how collective acting can depend on tipping points (or critical mass). Schelling segregation model also contains tipping points, however the term was named and popularized by Granovetter.

What the field of complexity science has to offer to the field of sociology is a sophistication of the methods of modeling the micro-macro link. In this dissertation a modest contribution is made by researching methods borrowed from the field of 
Table 1.1: Cooperation problem represented as a prisoners dilemma.

\begin{tabular}{c|cc} 
& Cooperate & Defect \\
\hline Cooperate & 3,3 & 1,4 \\
Defect & 4,1 & 2,2
\end{tabular}

complexity. The methods used in this dissertation are network science, game theory, statistical mechanics and economic complexity. In what follows we introduce how these methods are applied to substantial research topics.

\subsection{Part 1: Chapters 2 till 4}

The study of why people cooperate is older than many academic disciplines that study the problem of cooperation. Although questions regarding cooperation were asked long before, many regard the book titled Leviathan by Thomas Hobbes written and published in the year 1651 - as the starting point of the scientific inquiry (Hobbes, 1968; Christman, 2017). Cooperation is studied in many disciplines and different contexts. However, the problem of cooperation might be most simple and elegantly formulated by social dilemma games.

Social dilemmas are situations in which rational choices by individual actors lead to outcomes that are suboptimal compared to when the actors had cooperated (Raub et al., 2015; Buskens and Raub, 2013). Arguably the most famous social dilemma game is the prisoner's dilemma (Binmore et al., 2007). As can be seen in Table 1.1, in the prisoner's dilemma the players can choose to cooperate or to defect. The cooperation problem arises because defection is always the best response to both choices of the other player. Therefore, both players choose to defect. Both players would have had a higher payoff if both chose to cooperate. However, cooperation is not a best response strategy. This example is arguably the most simple way of formalizing a cooperation problem. Therefore, the prisoner's dilemma has been topic of debate for many decades. Many different solutions have been brought forward such as playing the game repeatedly (Axelrod and Hamilton, 1981). More recently, network structure also has been put forward as a possible solution to the cooperation problem formulated by the prisoner's dilemma (Raub and Weesie, 1990; Nowak and May, 1992; Szolnoki et al., 2008; Gracia-Lázaro et al., 2012). In this dissertation we study if network structure can help to overcome another type of social dilemma. 
Table 1.2: Payoff table of an asymmetric coordination game

\begin{tabular}{c|cc} 
& $\alpha$ & $\beta$ \\
\hline$\alpha$ & 2,1 & 0,0 \\
$\beta$ & 0,0 & 1,2
\end{tabular}

The social dilemma studied in this dissertation is known as the 'battle of the sexes' (BoS). In game theory, the 'battle of the sexes' represents a situation in which people like to agree on options in the first place, but some people prefer one option more than the other. This situation is called 'battle of the sexes' based on a suggested story in which a man prefers to go to a sports game, while a woman prefers to go to the theater. However, both would rather do something together than alone.

The 2-player version of 'battle of the sexes' is formalized in Table 1.2. In Table 1.2 , there is a row player and a column player. Both players can choose between two options, in this case named $\alpha$ and $\beta$. The payoff of the individual players is dependent on which option they choose. The two choice combinations create four payoff combinations, represented in the matrix in Table 1.2. In each of the four combinations the first entry in the matrix is the payoff of the row player and the second entry is the payoff of the column player. Both players have a higher payoff if they choose the same option compared to both choosing a different option. The row player has the highest payoff if both players choose $\alpha$ and the column player has the highest payoff if both players choose $\beta$.

A real life example of this asymmetric situation is choosing between two gaming consoles. This Christmas both Playstation and Xbox will release their new gaming console. Imagine, you and a friend want to buy one, but you prefer the Playstation and your friend the Xbox. You both prefer buying the same device over buying a different one, because this will allow you to play against each other over the internet. Studies show that when you have to make the decision which console to buy independently, you are most likely to end up choosing your own preferred option. In this case, you miscoordinate and this is the least favorable outcome for both of you (Cooper et al., 1989; Binmore et al., 2007). When you are allowed to communicate and go back and forth a couple of times you will probably converge on one of the two consoles (Duffy et al., 2017; De Kwaadsteniet et al., 2012; Lau and Mui, 2008). However, this can be frustrating for the player who did not end up with his or her most preferred option. 
Table 1.3: Example payoff table 3 player asymmetric 'battle of the sexes' game. The first entry is for player 1 , the second entry for player 2 , and the third entry for player 3 .

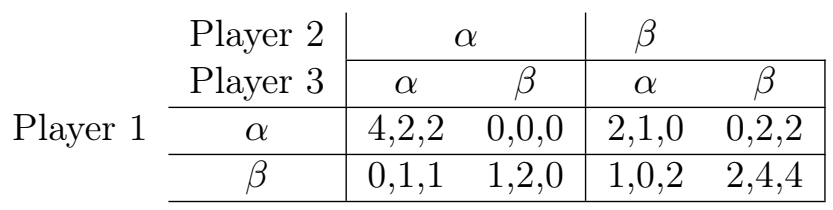

Table A12 illustrates the situation in which a third player is added who also has a preference for one of the two options. In the three player case, the situation changes and the payoff table is not balanced anymore. The intuitive explanation is that when three people have a preference for one of two options, there will always be a majority for one of the two options.

In Chapters 2 until 4, we study what happens when this $2 \times 2$ game is played by more than three players, where the players are connected by a network. In the network, the players are the nodes and they play the $2 \times 2$ game with other players who they share an edge with. The intuitive expectation is that network structure is relevant because the network will contain local majorities for one of the two options, as exemplified in Table A12. However, the network connects multiple local interaction points that may or may not have a majority for one of the two preferences. How these are connected might therefore be of vital importance for understanding how these decisions are established in larger groups. We hypothesize that the decision behavior in larger groups can be predicted from the mathematical properties of the network structure for an important part.

In Chapter 2 a simulation model is created. The goal of the simulation model is to create 'Laplace's demon' for these dynamics. By simulation, we create the system ourselves. We could describe the system by describing at every time point every choice made by each agent and why this choice was made. However, in order to do this, we need at least as much information to describe the system as the system produces. The goal of the simulation is to describe the system and predict the choice behavior with as little information as possible. This requires two steps. First, recognize if the system produces any patterns. Second, if the systems produces patterns, try to understand the causation of the patterns.

The expectation in Chapter 2 is that different network structures cause different choice patterns for battle of the sexes games with multiple players. In order to study whether different types of networks produce different patterns, we take 
the random 'Erdös-Rényi' network as a baseline. Because the baseline model is the most random of the chosen networks, we expect that no particular patterns of choice behavior will follow from these simulations. So many parameters are needed to describe the system, resulting in little reduction of information. However, we hypothesize that the less random the network structure, the more clear the patterns become. So in less random network structures, less parameters are needed to describe the system and the information can be summarized very effectively. In order to relate the patterns to the network structure, we vary a couple of properties of the networks. Next, we relate the resulting patterns to the mathematical properties of the different types of networks.

In Chapter 2 we show that the produced patterns in choice behavior are highly dependent on the mathematical properties of the network. In general, the less 'random' the structure of the links of the network are generated, the more precise the choice behavior can be predicted with a small set of parameters. The most important predictor variables are on the macro level how clustered a network is and on the micro level the degree centrality of the players. We show that all results are robust independent of network size.

In Chapter 3, the predictions made by the simulation model in Chapter 2 are tested in a computerized lab experiment. The goal is to empirically validate the theoretical model of Chapter 2. During the experiment, groups of 20 people played $2 \times 2$ games with other participants with whom they were connected through a network. The participants were asked to choose between the options 'blue' and 'yellow'. The participants could earn money if their choice corresponded with the choice of the participant(s) they were connected to. However, some participants received more money if they coordinated on 'blue', while others got more money when they coordinated on 'yellow'. Participants received nothing if one has chosen 'blue' and the other participant 'yellow'. So the game has both an element of coordination and an element of competition.

The results of the experiment are very much in line with the predictions of the computational model. As expected, the less 'random' the network structure, the more the experimental results are in line with the predictions of the computational models. The found correlation of behavior between the computational model and the experimental results is low for a random network, intermediate for clustered 'small world' networks, and high for centralized 'preferential attachment' networks. Furthermore, clustering of the network leads to higher heterogeneity of choices. And participants with higher degree centrality have more influence on the choices 
made in the network overall.

The results obtained in Chapter 2 are based on a computational model. In Chapter 4 we try to further generalize the results in Chapter 2 by adding analytical results. In order to obtain analytical results we make use of the ferromagnetic Ising model. We further generalize the ferromagnetic Ising model to represent the asymmetric interaction in the battle of the sexes. The asymmetric interactions are realized by allowing the parameter that in the original model represents the 'external magnetic field', to vary between nodes. Therefore, the magnetization can be one direction for one node and the other direction for another node. So, the asymmetric preferences of the battle of the sexes can be modeled by means of the magnetization of the individual nodes. We find that there are some differences in the equilibrium behavior between the computational model and the Ising model. With the Ising model we find a richer set of equilibria compared to the computational model. This finding actually strengthens our faith in the results obtained by the computational model, because these results seem to suggest that if we model coordination problem in Chapter 2 analytically, the results become stronger. One reason to suspect that the results become stronger when modeled analytically is that the update rule used in computational models are unlikely to find unstable equilibrium points. However, it should be noted that we where unable to formulate the Ising model such that it accurately corresponds to the results of the computational model in all networks considered.

\subsection{Part 2: Chapter 5}

In Chapter 5 we recognize that models of small groups with repeated interactions cannot fully explain social cohesion. Modern societies do not only function according to repeated interactions with acquainted people. The more complex a society becomes, the more daily life becomes defined by social interactions with people one is not acquainted with. Instead, people interact with people they have not met before everyday. Therefore, repeated interactions and the structure of one's social network can explain some variance of the cohesion problem, but not all of it. In Chapter 5, we argue that social interactions are for an important part shaped by a division of labor and regulatory institutions. We go back to a classical sociological theory known as 'organic solidarity', that has 'long been forgotten in modern debate', but is making a revival in recent studies (Portes and Vickstrom, 2011; Abascal and Baldassarri, 2015). 
Phenomena such as social cohesion, cooperation and trust certainly can be established via the repeated interactions of small cohesive groups. However, in modern societies these interactions are embedded in a larger societal structure. The larger societal structure meaning the way a society is organized by its formal and informal institutions. So with 'institutions' we mean institutions in the broad sense, defined as 'stable, valued, recurring patterns of behavior' (Huntington, 2006). The patterns of behavior can be formal arranged and enforced, such as law and order, or self maintaining and informal, such as different forms of culture.

We argue that the effect of the division of labor, although an institution in itself, should be studied independently from other types of institutions. For instance, someone's job causes a powerful (economic) embeddedness that forces people to position themselves against others on a day to day basis. For instance, when a customer enters a shop, the social roles are immediately clear. Both the shop owner and the customer have to maintain a certain social standard to maintain their economic position. Therefore, economic relations help define social order. How these relations are structured can therefore be of vital importance to various social mechanisms that extend beyond the economic realm.

In a recent study, authors inspired by Durkheim's theory on organic solidarity, theorize on the relevance of this theory in current debate (Portes and Vickstrom, 2011; Durkheim, 1893). They write that organic solidarity is dependent on three conditions, namely:

1. (Economic) Diversity among members of a society

2. Strong coordinating institutions

3. A complex division of labor (specialization).

The influence of formal institutions on positive outcomes for society, such as cooperation and trust, is a widely studied subject and topic of ongoing debate (Fehr and Gachter, 2000; Kosfeld et al., 2009; Gürerk et al., 2006; Lo Iacono, 2019; IOS, 2019). However, the effect of a complex division of labor and economic diversity has not yet been tested empirically.

In Chapter 5 we use recent methods from the field of economic complexity that aim to quantify (economic) diversity among members of a society and the specialization of an occupation. We use the obtained variables and test how they relate to selfreported generalized trust. We argue that generalized trust cannot be explained by 
repeated interactions between individuals. In the used survey question measuring generalized trust the respondent is asked: 'Generally speaking, would you say that most people can be trusted or that you can't be too careful in dealing with people?'. The question asks if people in general can be trusted and not only people one is acquainted with or had previous interactions with. Therefore, in order to explain generalized trust we need to turn to other types of explanations, such as organic solidarity. In Chapter 5 we show that there is a large effect of both occupational diversity as well as occupational complexity on whether the respondents answer positively on the generalized trust question.

\subsection{Future research}

In general, the field of 'games on graphs' is slowly getting saturated on the modeling side. With that I mean that I do not expect major field changing discoveries to happen. Although important nuances can still be made. In Chapters 2 and 3, the agents and people try to coordinate using local information only. An interesting addition would be to study whether global information could further help to facilitate the coordination process.

In Chapter 4, we where unable to find a single set of parameters such that an Ising model accurately represent the results of the computational model of Chapter 2 in all cases. Instead, the parameters of the Ising model depend on the network type for approximating the computational results. In terms of parsimony, it would be interesting to find the microscopic parameters such that the Ising model can mimic the behavior of the computational model always. If such microscopic parameters exist, they can be used to generalize predictions for different types of networks.

On the empirical side of on the subject of 'games on graphs' there are still many opportunities. Particularly, field experiments on the effect of network structure on coordination processes and/or social dilemma situations are lacking. The models can be used as a theoretical framework for deriving hypotheses on empirical situations. The other way around, empirical studies can be used in validating and readjusting the parameters of existing models. Managing and coordinating are jobs people get paid to do, so a field experiment testing current models (or theories) should not be hard to find.

On further understanding the theory of organic solidarity there is potentially much to gain. The results indicate there is an effect of a division of labor on trust. However, the difficulty with the theory of organic solidarity is that there are problems 
with endogeneity which need attention: economic interdependence induces trust, but trust in return leads to people willing to collaborate more. Furthermore, both dependent and independent variables are imprecise measures that can benefit from empirical clarity and theoretical rigor. How economic embeddedness causes people to trust more is an open question. In order to improve our understanding of these mechanisms, new micro models need to be developed that directly link economic embeddedness to individual behavior. Furtermore, we need to theorize about how generalized trust (or organic trust) differs from communitarian or in-group based types of trust. Next, we need to formulate hypotheses on when to expect communitarian trust mechanisms to take happen and when organic trust mechanisms happen and test these hypotheses empirically.

Maybe the biggest question left unanswered in this dissertation is why the theory of organic solidarity has been largely forgotten in modern debate? On the one hand, the theory seems like one of the most simple and elegant explanations of human cooperation in the history of sociology. On the other hand, the theory might be so heavily underdetermined that in fact we never stopped researching it. I think the theory has established the pattern, we now just need to find the origin. 



\section{Part I}

Cohesion and polarization in asymmetric coordination 



\title{
Chapter 2
}

\section{Network effects on coordination in asymmetric games ${ }^{1}$}

\begin{abstract}
Network structure can have an important effect on the behavior of players in an iterated 2x2 game. We study the effect of network structure on global and local behavior in asymmetric coordination games using best response dynamics. We find that global behavior is highly dependent on network topology. Random (Erdös-Rényi) networks mostly converge to homogeneous behavior, but the higher the clustering in the network the more heterogeneous the behavior becomes. Behavior within the communities of the network is almost exclusively homogeneous. The findings suggest that clustering of networks facilitates self-organization of uniform behavior within clusters, but heterogeneous behavior between clusters. At the local level we find that some nodes are more important in determining the equilibrium behavior than other nodes. Degree centrality is for most networks the main predictor for the behavior and nodes with an even degree have an advantage over nodes with an uneven degree in dictating the behavior. We conclude that the behavior is difficult to predict for (Erdös-Rényi) networks and that the network imposes the behavior as a function of clustering and degree heterogeneity in other networks.
\end{abstract}

\footnotetext{
${ }^{1}$ This chapter is published as: Broere, J., Buskens, V., Weesie, J. \& Stoof, H. (2017). Network effects on coordination in asymmetric games. Scientific reports, 7(1), 17016. Broere wrote the manuscript, did the simulations and analysis. Broere, Buskens, Weesie and Stoof contributed to the idea development and manuscript writing. All authors discussed the results and reviewed the manuscript. We thank Rense Corten, Jonas Haslbeck, Oisin Ryan and Merel van Herpen for their comments on earlier drafts of this manuscript.
} 


\subsection{Introduction}

Coordinating interdependent behavior when actors have different interests can be extremely difficult. These types of situations can be represented by an asymmetric 'battle of the sexes' game as shown in Table 2.1. Table 2.1 represents a situation in which two actors have a choice between $\alpha$ and $\beta$. Coordination on the same choice yields the highest payoff, but the actors differ in their preference for either $\alpha$ or $\beta$. Analyzing this situation, it becomes clear that the Nash equilibria seem either unfair, because one actor is better off than the other, or inefficient in the case of a mixed Nash equilibrium (Binmore et al., 2007). Therefore, it is difficult to make behavioral predictions on the choices of players in these types of situations. The coordination problem is potentially larger when there are more than two actors involved. However, in a three player game, the behavior is already easier to predict when two players have a preference for $\alpha$ and one has a preference for $\beta$. The most likely Nash equilibrium is for all players to play $\alpha$, because this yields the highest utility for the two $\alpha$ players, independent of what the $\beta$ player does. In games with more than three players, the behavioral prediction is dependent on the interaction structure. When the interaction structure is represented as a network, there are local majorities of actors that have a preference for one of the equilibria. Therefore, it seems plausible that the network structure is crucial for understanding an asymmetric coordination problem with multiple actors. In this chapter we study the influence of network structure on global and local behavior in iterated asymmetric coordination games.

Situations in which interdependent actors have to coordinate behavior with unequal preferences are wide-spread. The classic 2 by 2 example is the situation of a man and a woman who have to coordinate their evening out without means of communication. The man has a preference to go to a football match and the woman prefers to go to the opera, however, they still prefer to go to the same event over going to the events alone. It is very hard to coordinate this problem with no means of communication. There are also contexts in which the group and interaction structures are relevant. Think for instance about a school class

Table 2.1: Payoff table of an asymmetric coordination game

\begin{tabular}{c|cc} 
& $\alpha$ & $\beta$ \\
\hline$\alpha$ & 2,1 & 0,0 \\
$\beta$ & 0,0 & 1,2
\end{tabular}


in a theme park that has to decide which attraction to go to next. Some of the children prefer to go to the roller coaster and others prefer to go to the water attraction. However, they also prefer going with their friends instead of going alone. It is therefore likely that a person with a preference for attraction $A$ will still go to attraction $B$ if the majority of his or her friends have a preference of attraction $B$. So, whether a preferred option is also the option which yields the highest utility might be dependent on the preferences of others in the network and the interaction structure. Similar dynamics can be expected when people have to choose between (operating) systems on their phone, computer or game consoles. For some applications it is necessary that your friends have the same system, so coordinating on the same system as your friends might yield more utility than choosing your personal favorite system.

A classical game theoretical example of modeling asymmetric situations is the socalled 'battle of the sexes' (BoS). BoS is a special case of a coordination game with two pure-strategy asymmetric equilibria and one mixed strategy equilibrium. As presented in Table 2.1, the players differ with respect to their preferences over the equilibria, but coordinating on the same equilibrium is still preferred over miscoordination. The equilibria of this game have been widely studied theoretically and empirically in both one shot and repeated versions of BoS (Binmore et al., 2007; Cooper et al., 1989). However, it is not at all clear for the network version of BoS to what equilibrium the game will converge, if at all. Emerging equilibrium behavior is likely dependent on the initial conditions and the spatial structure of the network.

Some research has been done on 'battle of the sexes' types of games in the context of spatially distributed interactions in both theoretical and experimental settings. A great amount of work on spatial BoS games is done by Alonso-Sanz, mostly in the context of homogeneous spatial structures such as cellular automata (AlonsoSanz, 2011; Alonso-Sanz, 2012a; Alonso-Sanz, 2012b). Some interesting results include the ability of self organization by means of forming homogeneous clusters in the spatial structure. Furthermore, Hernandez, Muñoz-Herrera and Sánchez (2013) introduce a model to analyze the Nash equilibria of an asymmetric game on a network. They find that on Erdös-Rényi networks situations equilibria exists where all players can choose there preferred action when the heterogeneity is high in the network, whereas players tend to coordinate on the other action when they are in a clear minority situation. In related work the influence of the strength of preferences is studied (Hernández et al., 2017). The higher the dif- 
ference in preferences the harder it is to reach coordination. However, when the difference in the preferences are small, full coordination is always an equilibrium. Mäs and Nax (2016) studied the response behavior of human subjects in two fixed networked coordination games in an experimental setting. An interesting finding is that 96 percent of the decisions followed a myopic best response pattern. Other related studies show the effect of asymmetries on cooperation in (weak) Prisoner's Dilemma games (Wang et al., 2014; Szolnoki and Perc, 2014; Amaral et al., 2016). An important result is that asymmetry introduced by payoff heterogeneity or mixed games has a favorable effect on cooperation. The main difference between our study and the studies above is that we study the effect of network structure on behavior, both on the local and global level and we derive predictions from the network structure.

To the best of our knowledge, no studies have been performed on the influence of the spatial structure of a network on the equilibrium behavior in a BoS game. We believe that studying a BoS game is particularly interesting because these games provide us with information on which types of nodes end up in their preferred equilibrium and which do not, dependent on the spatial position. If network structure is of any influence, some nodes should have more powerful positions in the sense that in their position in the network they more easily coordinate on its preferred behavior. Therefore, network structure can be of vital importance in understanding asymmetric coordination problems with multiple actors.

Network structure is often found to be a crucial concept in understanding many phenomena such as virus spreading, percolation, social cooperation and information diffusion (Szabó and Fath, 2007; Albert and Barabási, 2002; Watts and Dodds, 2007; Stegehuis et al., 2016). Many studies have shown that differences in network topology can lead to wildly different behavior. These studies often focus on global network effects, such as cooperation in a Prisoner's Dilemma game, or just local effects, such as identifying influential spreaders in diffusion models (Santos et al., 2006; Freeman, 1977; Kitsak et al., 2010). We argue that global and local dynamics are inevitably dependent on each other. Considering that network structure has an effect on global level dynamics, there must be an effect on the local level as well and vice versa.

Game theory is an often used method of modeling network dynamics (Szabó and Fath, 2007; Albert and Barabási, 2002). Game theory is a set of analytical tools to represent interdependent situations between agents, designed to model and make predictions on decision making. In the networked game, agents play games with 
Table 2.2: Possible payoff situations in BoS on a network, where $0<S<1$

(a)

\begin{tabular}{c|cc} 
& $\alpha$ & $\beta$ \\
\hline$\alpha$ & $1, S$ & 0,0 \\
$\beta$ & 0,0 & $S, 1$
\end{tabular}

(b)

\begin{tabular}{c|cc} 
& $\alpha$ & $\beta$ \\
\hline$\alpha$ & 1,1 & 0,0 \\
$\beta$ & 0,0 & $S, S$
\end{tabular}

(c)

\begin{tabular}{c|cc} 
& $\alpha$ & $\beta$ \\
\hline$\alpha$ & $S, S$ & 0,0 \\
$\beta$ & 0,0 & 1,1
\end{tabular}

a subset of a population, represented by edges in a network. Many computational studies have shown that the spatial structure of a network can have an influence on the evolution of behavior in a game (Roca et al., 2009; Szabó and Fath, 2007; Buskens and Snijders, 2015). Santos et al. (2006) compare complete, small-world and scale-free networks on cooperative behavior. They show that heterogeneity of the degree distribution has an important influence on the asymptotic density of cooperators in symmetric games such as Stag-Hunt and Prisoner's Dilemmas. Tomassini and Pestelacci (2010) show that the presence of clusters of highly connected nodes in a network has a positive impact on cooperation. However, too much clustering can lead to so-called 'topological traps' (Roca et al., 2010). These traps can prevent cooperation from spreading uniformly through the network. Most of these studies focus on the effect of the spatial structure on the behavior of symmetric games, such as symmetric Prisoner's Dilemmas and symmetric coordination games. However, interesting situations can also arise in asymmetric games.

\subsection{Methods}

We utilize a 'battle of the sexes' coordination game to represent actors with their different preferences. Assuming that the behavior of the most influential or powerful network positions are more likely to converge to their preferred equilibrium than the less influential or powerful network positions, spatial effects can be understood by the probability of a given node in a network to end up in its preferred equilibrium, irrespective of other initial conditions.

In Table $2.2 \mathrm{a}$ the utility matrix of the $2 \times 2 \mathrm{BoS}$ game is presented. The game is modeled such that $0<S<1$. In this case the row player has the highest payoff when both players choose $\alpha$ and the column player has the highest payoff when both players choose $\beta$. Coordinating on the same behavior is more rewarding than miscoordination. The $2 \times 2$ game can be mapped on a network by pairwise 
interactions between nodes who share an edge. In this network, every node has a preference for either $\alpha$ or $\beta$, so is a row or a column player. When nodes play pairwise interactions against multiple nodes on a network, three situations can occur. The first situation is a pairwise interaction between two nodes who differ on their preference for $\alpha$ or $\beta$, as already described in Table 2.1 and again in Table 2.2a. In the second situation, two nodes with the same preference for $\alpha$ pairwise interact, as shown in Table $2.2 \mathrm{~b}$. In the third situation, two nodes with the same preference for $\beta$ interact, as shown in Table 2.2c. In the $2 \times 2$ case, coordination is difficult in the first situation, but rather obvious in the second and third situation.

A potential extra difficulty is added when nodes have to coordinate with multiple nodes at the same time in a network, while having to choose for each connection the same behavior (Easley and Kleinberg, 2010a). The total utility of a node depends on the returns of multiple interactions at the same time. Say node $i$ has $d$ neighbors and a preference for playing $\alpha$. A fraction $q$ of the neighbors play $\alpha$ and the fraction $(1-q)$ plays $\beta$. If node $i$ chooses $\alpha$ the payoff will be $q d$ as the utility will be 0 for miscoordinating with nodes who play $\beta$. If node $i$ chooses $\beta$ the payoff will be $(1-q) d S$. Assuming node $i$ knows $q$, node $i$ will choose $\alpha$ if

$$
q d \geq(1-q) d S
$$

By rearranging the terms,

$$
q \geq S /(1+S)
$$

it becomes clear that the choice for $\alpha$ or $\beta$ is dependent on the fraction of neighbors that play $\alpha$ or $\beta$. However, the game is played multiple rounds and the nodes don't know in advance what the strategy is that their neighboring nodes will play.

In each round the nodes update their belief on what strategy yields the highest payoff, $\alpha$ or $\beta$, by means probabilistic dynamic in which the behavioral propensity changes towards the best response. Let $i=1 \ldots N$ be the nodes in the population. Let $s \in\{\alpha, \beta\}$ be the strategy of node $i, \pi_{i}$ the payoff of node $i$ and $\pi_{i}^{*}$ the payoff when the alternative strategy would have been played. Then, the probability $p_{s, i}^{t}$ that a strategy $s$ is played in round $t$, given the probability $p_{s, i}^{t-1}$ that a strategy $s$ is played in round $t-1$ equals;

$$
p_{s, i}^{t}= \begin{cases}p_{s, i}^{t-1}+0.1 & \text { for } \pi_{i}^{t-1} \geq \pi_{i}^{*, t-1} \\ p_{s, i}^{t-1}-0.1 & \text { for } \pi_{i}^{t-1}<\pi_{i}^{*, t-1},\end{cases}
$$

where $p_{s, i}^{t}$ is the probability that strategy $s$ is played at some time $t$ by node $i$ (Buskens and Snijders, 2015). So, at every time $t$ each node updates the probability to play $\alpha$ or $\beta$ towards a myopic best response reply strategy. If the best reply 
at time $t-1$ would have been $\alpha$, the probability of playing $\alpha$ at time $t$ increases compared to time $t-1$. If the best reply at time $t-1$ would have been $\beta$, the probability of playing $\alpha$ at time $t$ decreases at time $t$ (simultaneously the probability to play $\beta$ increases). The probabilities are naturally bounded by the values 0 and 1 . We choose this probabilistic response behavior because this prevents the problem of non-convergence, compared to the deterministic myopic best response behavior. However, the results and dynamics are very similar to the deterministic version of myopic best response.

\subsubsection{Simulation Design}

We perform a computational study in which actors play 2 x 2 games against their neighbors represented by the nodes and edges of a network. Three types of networks are considered, namely random Erdös-Rényi (ER) networks, small-world (SW) networks, and preferential attachment (PA) networks. The ER-networks are generated using the $G\left(N, p_{e r}\right)$ Erdös-Rényi model, where $N$ is the number of nodes in the graph and $p_{e r}$ the probability for drawing an edge between two arbitrary nodes. We choose $N=20$ and $p_{\text {er }}=0.2$ in our simulation. The SWnetworks are generated using the Watts-Strogatz algorithm (Watts and Strogatz, 1998). The algorithm starts with a one-dimensional lattice consisting of $N=20$ nodes. Each node is connected with two neighboring nodes by an edge. The edges are rewired randomly with probability $p_{s m}$. SW-networks are known to have short average path lengths and high clustering. The clustering decreases with the value of $p_{s m}$. The higher the value of $p_{s m}$, the more the network will resemble the Erdös-Rényi model in terms of clustering (Watts and Strogatz, 1998). In order to vary the amount of clustering within SW-networks, we vary $p_{s m}$, where $p_{s m} \in\{0.05,0.1,0.15,0.2,0.25\}$. The PA-networks are generated by the algorithm proposed by Barabasi and Albert (1999). The algorithm starts with $m_{0}$ nodes. With each iteration one new node with $m$ edges adds on to the existing nodes with probability $p_{p a}$, where $p_{p a, i}=k_{i} / \sum_{j} k_{j}$, and where $k_{i}$ is the degree of node $i$ and the sum is made over the previously added nodes. This process continuous until the network consists of $N=20$ nodes. We choose $m=2$ in order to keep the density of the network equal to the other types of networks. We choose $N=20$ for all networks in this study because with this size the relative influence of one node on the global behavior can still be substantial, while this size is big enough to guarantee the complexity of behavior in the network. Results for larger network sizes will be discussed in Figure A3 till Figure A5 and, Table A2 and Table A3 of the appendix. We only include connected graphs for all network types throughout 
the whole simulation.

All networks are generated with size 20 of which 10 nodes have a preference for $\alpha$ (row players in Table 2.2a) and 10 nodes have a preference for $\beta$ (column players in Table 2.2a). The constraint of $10 \alpha$ and $10 \beta$ players is imposed to maximize the coordination problem. The preferences of the nodes are randomly assigned. In the simulation a 1000 ER-networks, SW-networks and PA-networks are generated, thus 3000 networks in total. For each network the game is played 100 times.

After the networks are initialized the iterated game starts. In each iteration a node has to choose between $\alpha$ and $\beta$. The total obtained utility is a function of the actions of the neighboring nodes, given by the utility matrix in Table 2.2. We chose $S \in\{0.9,0.7,0.5\}$. If $S$ becomes smaller, the incentive to deviate from one's preferred behavior becomes smaller as well. In the remainder of this paper we only discuss results for the single case $S=0.9$ because this value maximizes the dynamics in the system. Results for other values of $S$ can be found in Figure A6, Figure A7 Table A4 and Table A5 of the appendix. Initially, at $t=0$, each node plays its preferred option with probability 1 . After each round the probabilities to play $\alpha$ or $\beta$ are updated by means of the response decision rule described in Equation 10. The game continues until none of the nodes changes probabilities anymore, so all probabilities are either 0 or 1 to play $\alpha$. The iterations run with a minimum of 10 and a maximum of 100 iterations. After each game several variables are saved. These include the initial conditions, several node and network characteristics and the equilibrium state of the node and the network. All files necessary to replicate the simulation can be found on the first author's github page: https://github.com/JJBroere/Network-effects-oncoordination-in-asymmetric-games.

\subsubsection{Variables}

The goal of this study is to infer how equilibrium behavior depends on the initial conditions of the networks as well as the power of an individual node to determine its equilibrium behavior given its position in the network. The first dependent network level variable is the proportion of nodes playing $\alpha$ in the network. A proportion of 1 indicates that all nodes in the network play $\alpha$ and a proportion of 0 indicates all nodes in the network play $\beta$. We define heterogeneity of the behavior as the variance of $\alpha$ behavior in the network;

$$
h\left(p_{\alpha}\right)=\operatorname{var}\left(p_{\alpha}\right)=p_{\alpha}\left(1-p_{\alpha}\right)
$$


Table 2.3: Number of neighboring nodes required for a local majority

\begin{tabular}{l|cccccccc}
\hline Degree centrality & 1 & 2 & 3 & 4 & 5 & 6 & 7 & 8 \\
Number of neighboring nodes needed & 1 & 1 & 2 & 2 & 3 & 3 & 4 & 4 \\
Percentage of neighboring nodes needed & $100 \%$ & $50 \%$ & $67 \%$ & $50 \%$ & $60 \%$ & $50 \%$ & $57 \%$ & $50 \%$ \\
\hline
\end{tabular}

where $p_{\alpha}$ is the proportion of nodes playing $\alpha$ in the network.

The second dependent variable is the dichotomous variable Preferred, indicating whether a node ends up in the equilibrium of preference after the game has converged. Third, we define Power of a node as the probability of a node to converge to its preferred equilibrium independent of the distribution of preferences. So, if different distributions of preferences on a network are played, what is the proportion of times a node converges to the preferred equilibrium given its position in the network. 100 different distributions of preferences per network will be played. Power is thus, the aggregate variable of Preferred.

In addition, we consider several independent variables that could be indicative of the equilibrium behavior of the game. The first set of independent variables are centrality measures. From common sense it might be expected that the most central nodes in a network are the most powerful. A wide range of centrality measures have been developed to account for different aspects of centrality in a network. In this paper four different centrality measures are considered, namely degree centrality, eigenvector centrality, betweenness centrality and closeness centrality. See the supplementary methods section of the appendix for the formal definitions of the centrality measures used in this chapter. Furthermore, we add a dummy variable indicating whether the degree centrality of a node is even or uneven. As can be derived from Equation 5.3, where $S=0.9$, the fraction of neighbors playing the preferred behavior of node $i$ in order for node $i$ to also play its preferred behavior is different for nodes with an even degree compared to an uneven degree. As presented in Table 2.3, the fraction where $q>S /(1+S)$, thus $q>0.474$ is always $50 \%$ for nodes with an even degree centrality while the fraction is bigger for an uneven degree centrality. The difference decreases as the degree centrality increases.

Secondly, we look at the influence of clustering on the behavior in the network. Clusters, modules or communities are closely connected subgraphs within a network. As shown by Roca et al. (2010), clustering can be an important predictor of whether or not behavior will spread uniformly through a network. Alonso-Sanz (2011) shows how behavior self-organizes in homogeneous (agreement) clusters 
when played on cellular automata. We expect that the self-organizing behavior of preferences will be influenced by network characteristics and more specifically the clustering of the network. This clustering effect might be even more relevant when groups of nodes with the same preference are clustered together.

In this study we use the Walktrap algorithm as described by Pons and Latapy (2006) to identify communities in a network. This algorithm performs a random walk on the graph. The main idea is that in clustered networks the random walk is more likely to remain in the same community than leave the community because of higher connectedness within the community. For every node in the network the random walk is used to compute a 'distance' between all pairs of nodes. The computed distance is used to find the partition that maximizes the modularity.

We also use the modularity to quantify how clustered a network is. Modularity is the fraction of edges within one module minus the expected fraction of edges if the edges where distributed at random (Newman, 2006). Modularity of a network is defined as;

$$
Q=\frac{1}{2 N} \sum_{i j}\left[A_{i j}-\frac{k_{i} k_{j}}{2 N}\right] \delta\left(c_{i}, c_{j}\right),
$$

where $N$ is the number of nodes, $\boldsymbol{A}$ is the adjacency matrix, $k$ the degree of a node, $c$ is the number of the community a node belongs to as identified by the Walktrap algorithm described above and $\delta(x, y)$ is 1 if $x=y$ and 0 otherwise.

The third set of independent variables are related to the initial conditions of the network. The preferences of the nodes are randomly assigned on the network. In order to evaluate how the equilibrium behavior is related to the local distribution of preferences, for each node two variables are saved. First, for every node, the percentage of neighbors with the same preference is saved, defined as;

$$
L_{i}^{n}=\frac{\sum_{j} A_{i j} \delta\left(p r_{i}, p r_{j}\right)}{\sum_{j} A_{i j}},
$$

where $\boldsymbol{A}$ is the adjacency matrix, $p r$ is the preference of the node (row or column player).

Secondly, the percentage of nodes with the same preference within the same community is saved, defined as;

$$
L_{i}^{c}=\frac{\sum_{j} \delta\left(c_{i}, c_{j}\right) \delta\left(p r_{i}, p r_{j}\right)}{\sum_{j} \delta\left(c_{i}, c_{j}\right)},
$$


Table 2.4: Descriptives of the dependent and independent variables.

\begin{tabular}{lccccc}
\hline \hline Statistic & $\mathrm{N}$ & Mean & St. Dev. & Min & Max \\
\hline Proportion $\alpha$ & 300,000 & 0.500 & 0.337 & 0 & 1 \\
Heterogeneity & 300,000 & 0.146 & 0.104 & 0 & 0.250 \\
Preferred & $6,000,000$ & 0.640 & 0.478 & 0 & 1 \\
Power & 60,000 & 0.640 & 0.108 & 0.2 & 1 \\
Eigenvector centrality & 60,000 & 0.523 & 0.245 & 0.004 & 1 \\
Betweenness centrality & 60,000 & 0.101 & 0.108 & 0 & 1 \\
Degree centrality & 60,000 & 0.191 & 0.115 & 0 & 1 \\
Same preference cluster & 60,000 & 0.471 & 0.254 & 0 & 1 \\
Same preference neighbors & 60,000 & 0.474 & 0.322 & 0 & 1 \\
Modularity & 3,000 & 0.313 & 0.069 & 0.112 & 0.518 \\
\hline
\end{tabular}

where $c$ is the community a node belongs to as identified by the Walktrap algorithm described above.

\subsubsection{Descriptive statistics}

In Table 2.4 the descriptive statistics of all dependent and independent variables are presented. The dataset contains of 3,000 networks, information about 20 nodes and 100 starting configurations per network, leading to 6,000,000 'observations'. Figure A1 in appendix of this dissertation shows that the variance of the variable Preferred caused by the stochasticity of the response dynamics is limited when the same preference distribution is played on a network multiple times. The Figure A2 in appendix shows that the estimates of Power are stabilized after 100 different initial conditions.

\subsection{Results}

To get a first impression on how the equilibrium behavior is related to network type we first look at the global behavior of the networks. In Figure 2.1 histograms are shown for the proportion of $\alpha$ behavior after convergence of the dynamics. As can be seen in Figure 2.1, most frequently the behavior in ER-networks converges to either uniform $\alpha$ or uniform $\beta$ behavior. The behavior for SW-networks are shown for different values of the rewiring probability $p_{s m}$. When $p_{s m}=0.25$ the behavior is mostly uniform $\alpha$ behavior or uniform $\beta$ behavior, closely resembling the 

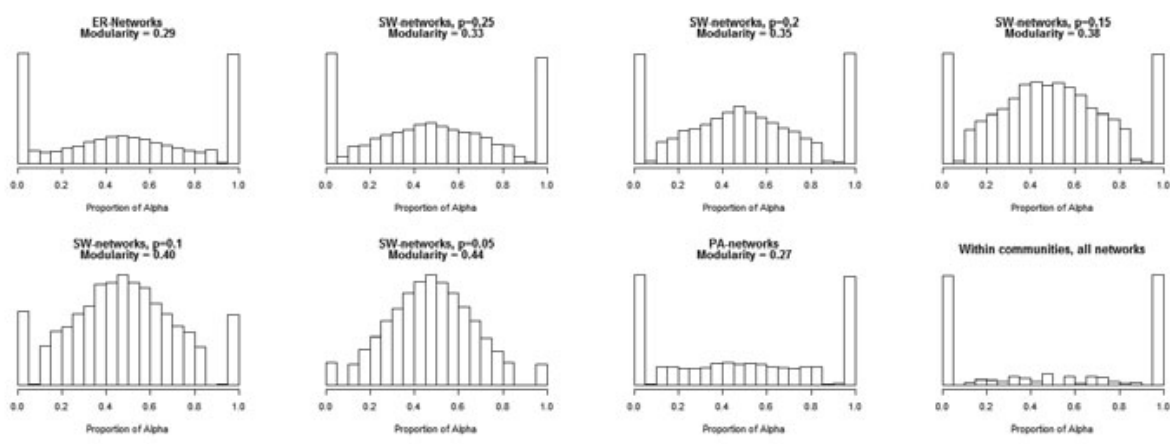

Figure 2.1: Proportion of $\alpha$ played in a network after convergence for ER-networks, SW-networks with rewiring probability 0.25 , SW-networks with rewiring probability 0.2 , SW-networks with rewiring probability 0.15 , SW-networks with rewiring probability 0.1 , SW-networks with rewiring probability 0.05 , PA-networks and within communities of all networks.

outcome of the ER-Network. However, the lower the value of $p_{s m}$, and therefore the higher the level of modularity/clustering, the more heterogeneous the behavior becomes. The behavior in SW-networks with $p=0.05$ seldom converges to uniform behavior. PA-networks have a low modularity and the behavior is mostly homogeneous. This trend can be described by the Pearson correlation between the heterogeneity of behavior in the network and the modularity of the network, which is $r=0.49$. In the bottom right histogram of Figure 2.1 the behavior is shown within communities of all networks in the data. The convergence behavior within communities of networks is almost exclusively homogeneous in all networks.

In Figure 2.2, kernel regression plots are shown for the dependent variable Preferred. Kernel regression is a non-parametric technique of estimating the conditional expectation of a random variable. The conditional expectation is computed by a locally weighted average given some kernel as a weight function. The kernels in Figure 2.2 are estimated with a box kernel and bandwidth $=0.5$. The two predictors are the proportion of adjacent neighbors that have the same preference as shown in the left part of Figure 2.2 and the proportion of nodes that have the same preference in the same community as shown in the right part of Figure 2.2. The plots indicate a strong relation between the clustering of the preferences and the expected probability that a node behavior converges to its preferred behavior. There seems to be a clear tipping point for the effect of the percentage of neighboring nodes with the same preference where there is a local majority of preferences. 

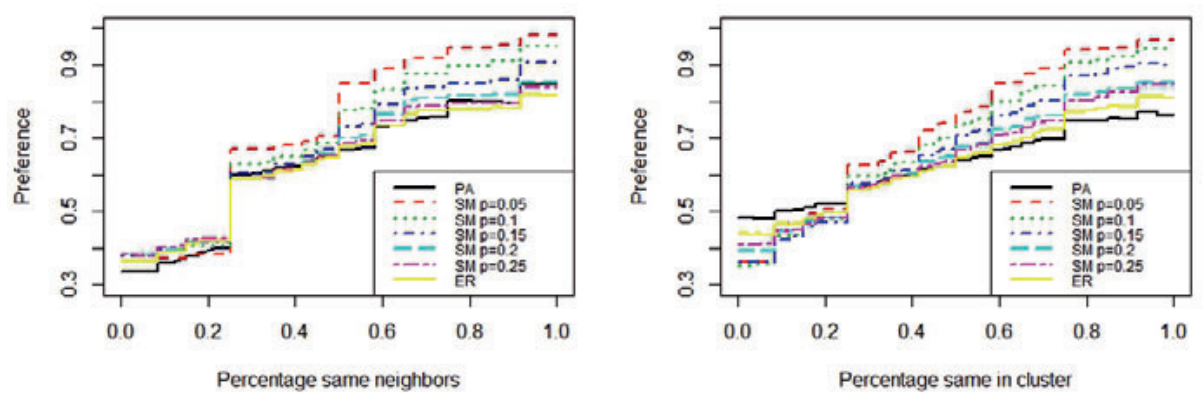

Figure 2.2: Kernel regression plot, dependent variable preference as a function of, left the fraction of same preference neighbors, right the fraction of same preference nodes in the community.

This relation clearly is stronger for the more clustered SW-networks compared to the ER-networks and PA-networks, indicating that the local interactions become more important in more clustered networks.

In Figure 2.3 the density of the variable Power is plotted for ER, SW and PAnetworks. The higher the proportion the more power a node has to determine its equilibrium behavior given its position in the network. A proportion of 0.5 indicates that the behavior is random and there is no association between the nodes spatial position and its equilibrium behavior. If the proportion is 1 , the node always converges to the preferred equilibrium and has maximum power to determine its equilibrium behavior. As can be seen in the left part of Figure 2.3, for ER-networks the density roughly follows a normal distribution with a mean of 0.618. The density of Power in PA-networks is comparable to the density of ER-networks with a mean of 0.637. On the right of Figure 2.3 the densities of SWnetworks for different values of $p_{s m}$ are plotted. The lower the value of $p_{s m}$ the more the density shifts to the right, indicating that there are more powerful nodes in SW-networks with lower value of $p_{s m}$. Because more clustered networks have more heterogeneous behavior, more nodes will be able to choose their preferred behavior.

In Table 2.5 the OLS-regression results with node Power as dependent variable are presented. Interactions between all variables are included in the model. To obtain the relevant predictors we used cross validated backward model selection 

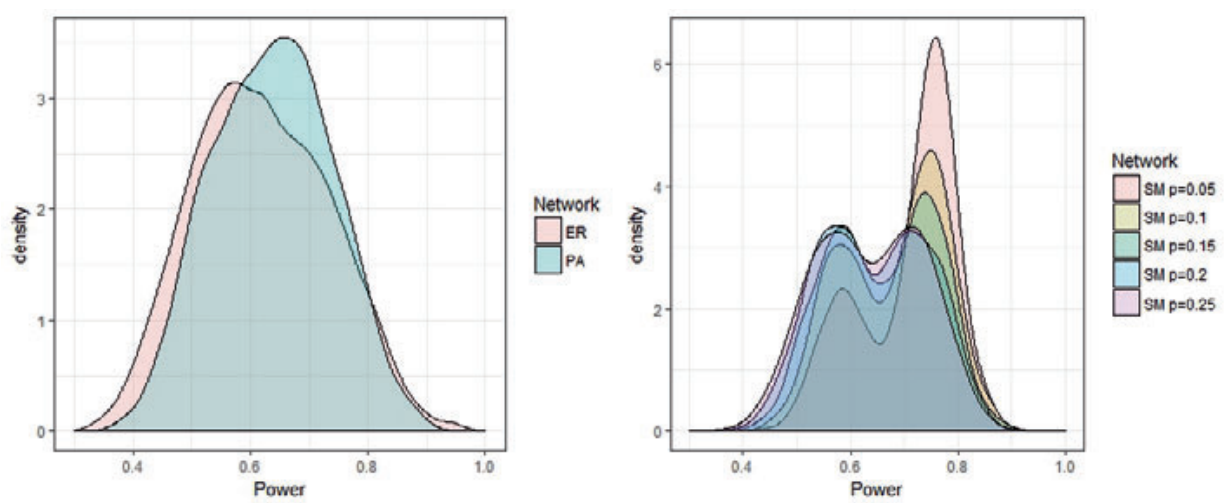

Figure 2.3: Density of node Power for random ER, Small World and PA-networks

based on a minimum difference of 0.01 in the $R^{2}$ of the model. There are two types of variance in the data, namely the within network and the between network variance. As shown in Table 2.5 the inter class correlation (ICC) does not exceed 0.022 for SW, is 0.062 for PA-networks and is 0.091 for ER-networks. We decided to ignore the between-level variance since most of the variance is on the withinlevel. The explained variance is 62.8 percent for ER-networks, 62.2 percent for PA-networks and always more than 70 percent for SW-networks.

Regression results indicate that an Even degree centrality is an important predictor for node Power in all types of networks. For all networks the predicted score for a nodes power increases around 0.16 , indicating a 16 percent higher probability to converge to its preferred behavior when a node has an even degree centrality. For nodes with an even degree it's easier to obtain a local majority compared to nodes with an uneven degree as shown in Table 2.3. For PA-networks there is one other important predictor for node Power, namely degree centrality. Nodes with the highest degree centrality have a 34.4 percent higher predicted probability of percent to converge to its preferred behavior. Nodes with an even degree and a high degree centrality the predicted probability is 99.4 percent. This seems to indicate that the global behavior is predominantly dictated by a few influential nodes with high degree centrality. In SW-networks degree centrality is also an important predictor for node Power, the effect is weaker compared to PA-networks. The final model for ER-networks is a complicated model in which all centrality measures are important predictors and the model has difficult to interpret interactions. But it is clear that in ER-networks having a high degree is not the only centrality measure 
Table 2.5: Regression results, standardized, dependent variable Power.

\begin{tabular}{lccccccc}
\hline \hline & ER & SW $p=0.25$ & SW $p=0.20$ & SW $p=0.15$ & SW $p=0.1$ & SW $p=0.05$ & PA \\
\hline Even & 0.154 & 0.159 & 0.160 & 0.165 & 0.165 & 0.172 & 0.155 \\
DegC & & 0.210 & 0.197 & 0.191 & 0.166 & 0.155 & 0.315 \\
EVC & 0.025 & & & & & & \\
BetC & 0.964 & & & & & & \\
ClosC & -0.470 & & & & & & 0.452 \\
BetC:EVC & -0.839 & & & & & & \\
EVC:ClosC & 0.369 & 0.478 & 0.488 & 0.499 & 0.516 & 0.524 & 20,000 \\
Constant & 0.627 & 4,000 & 4,000 & 4,000 & 4,000 & 4,000 & 0.532 \\
\hline $\mathrm{N}$ & 20,000 & 0.726 & 0.714 & 0.739 & 0.740 & 0.757 & \\
$\mathrm{R}^{2}$ & 0.628 & 0.797 &
\end{tabular}

${ }^{*}$ Even $=$ variable indicating an even degree, $E V C=$ Eigenvector centrality, BetC=Betweenness centrality, DegC $=$ Degree centrality, Clos $C=$ Closeness centrality. ${ }^{*}$ Interaction in uncentered variables.

leading to high degree centrality.

All results together seem to indicate that in ER-networks the global behavior is mostly homogeneous, but difficult to predict since this behavior is dependent on multiple centrality measures at the same time. In PA-networks the behavior is also mostly homogeneous, however in this type of network the behavior is dictated by a few influential nodes with high degree centrality. In SW-networks with high clustering, degree centrality is also important, but the spread of behavior is limited by the a node's community, leading to heterogeneous global behavior. In all networks, nodes with an even degree have an advantage over nodes with an uneven degree, because it is easier for nodes with an even degree to obtain a local majority.

\subsubsection{Robustness}

In order to check the robustness of the results we also varied the network size, the network density and the value of $S$. The results can be found in Figure A3 till Figure A7 and Table A2 till Table A5 of the appendix of this dissertation. As can be seen in appendix A3, more clustered networks have more heterogeneous behavior also in larger networks, the differences become even stronger for larger networks. As can be seen in Figure A4 and Figure A5, when network density increases the heterogeneity of behavior becomes less because the modularity decreases as the density increases. As can be seen in Table A2 and Table A3, there is a dependency at the local level on the size of the network. First of all, the positive effect of an even degree centrality decreases as the average degree centrality increases, which can be expected by looking at Figure 2.3. Degree centrality is the main 
predictor in SW-networks and PA-networks, however the relative power of one node decreases in larger networks, as can be seen from the decreasing regression coefficients. Secondly, in larger networks with high clustering, centrality measures are no longer predictive of node power, because the centrality measures are with respect to the network as a whole, while the spread of behavior is limited by the clustering. In this case the position of a node within its community is probably more important than its position in the network as a whole. In Table A4 and Table A5 results for the values of $S=0.7$ and $S=0.5$ are shown. For $S=0.7$ the results are comparable to those of $S=0.9$. For $S=0.5$ nodes are less likely to change their behavior, because the fraction $q=0.5 /(1+0.5)=0.33$ is lower. This can be seen in the higher intercepts of the regression models and the lower effect of the predictors. However, it should be noted that even with $S=0.5$ the behavior within clusters is mostly homogeneous.

\subsection{Discussion}

In this paper we study the effect of network structure on global equilibrium behavior and the behavior of individual nodes in asymmetric 'battle of the sexes' games. Looking at the heterogeneity of behavior in the network, we find that network topology has a large effect on the global equilibrium behavior of the networks. The heterogeneity of behavior is largely determined by the modularity of the network. These findings are independent of network size and even visible in other values of $S$. When there is a clear community structure in the network, coordinating on the same behavior within the community is more rewarding than coordinating with nodes outside the community, simply because a node has on average more edges in its community than outside its community. So, in networks with more clearly defined communities (high modularity), some communities can coordinate on one behavior while other communities coordinate on the other behavior, leading to more heterogeneity in behavior at the global level. The ratio of edges inside and outside the community is lower for networks with low modularity, so edges inside the community are almost equally important as edges outside the community. Therefore, nodes often coordinate on the same behavior throughout the whole network in networks with low modularity, in particular in ER-networks.

The same mechanism of clustering is at play when we look at the effect of the initial conditions. Whether a node converges to the preferred equilibrium is largely dependent on the preferences of the neighboring nodes. The probability that a node ends up in the preferred equilibrium can largely be predicted by the major- 
ity of preferences in the adjacent nodes or the nodes in the community the node belongs to. This relation is much stronger for more clustered networks. Clustering makes the relation to the direct neighbors and nodes within a cluster more important than the relation outside of the cluster. Together with the finding that high modularity limits the spread of uniform behavior, it can be expected that at some value of modularity the cluster topology becomes more important than the global level topology for the equilibrium behavior.

However, determining what the actual equilibrium behavior will be is not as simple as calculating the majority of preferences in a community. Not every node has the same weight or power to determine the behavior. Nodes with an even number of adjacent nodes have an advantage over nodes with an uneven number of adjacent nodes. A node with three neighbors needs two out of three to choose the same behavior, while a node with four neighbors needs two out of four neighboring nodes to choose the same behavior. Also, degree centrality plays an important role. PA-networks have low clustering and the degree distribution is known to have many nodes with low degree centrality and a few with high degree centrality. Therefore, the global behavior is mostly determined by a few nodes with high degree centrality. In SW-networks degree centrality is also important, but because of its clustering, the influence of a node with high degree on the global behavior is limited to the behavior of the community the node belongs to. For ER-networks global behavior depends in a less clear way on individual network positions. Because the clustering is low and degree distribution is less heterogeneous than PA-networks, often more complex and random network characteristics determine to which behavior the network converges.

It will be interesting if future research focuses on how the cluster (or meso) level is related to the local (or micro) level and global (or macro) level behavior. So, at what value of modularity is the meso-level topology more important for the dynamics than the global topology? In future research, we plan to test these computational results empirically on human subjects in an experimental study. 



\title{
Chapter 3
}

\section{An experimental study of network effects on coor- dination in asymmetric games ${ }^{1}$}

\begin{abstract}
Network structure has often proven to be important in understanding the decision behavior of individuals or agents in different interdependent situations. Computational studies predict that network structure has a crucial influence on behavior in iterated 2 by 2 asymmetric 'battle of the sexes' games. We test such behavioral predictions in an experiment with 240 human subjects. We found that as expected the less 'random' the network structure, the better the experimental results are predictable by the computational models. In particular, there is an effect of network clustering on the heterogeneity of convergence behavior in the network. We also found that degree centrality and having an even degree are important predictors of the decision behavior of the subjects in the experiment. We thus find empirical validation of predictions made by computational models in a computerized experiment with human subjects.
\end{abstract}

\footnotetext{
${ }^{1}$ This chapter is published as: Broere, J., Buskens, V., Stoof, H., \& Sánchez, A. (2019). An experimental study of network effects on coordination in asymmetric games. Scientific reports, $9(1), 6842$. Broere wrote the manuscript, did the analysis and programmed the experiment. Broere and Sánchez developed and executed the experiment. Buskens, Stoof, Sánchez contributed to idea development, experiment development and manuscript writing. All authors discussed the results and reviewed the manuscript. We thank Ignacio Tamarit and Pau Casanova for their help with the experiments. We thank Merel van Herpen for her comments on the manuscript.
} 


\subsection{Introduction}

Coordination problems are numerous in everyday life (Schelling, 1980; Crawford et al., 2008; Mehta et al., 1994). While avoiding collusion in traffic, meeting one another or making an exchange, coordination is a vital part of the success of the interaction. Coordination processes are therefore arguably fundamental to understanding the functioning of social, economic and biological systems. Game theoretical models are often used to model coordination problems with a strategic interdependence among actors. The success of this method has led to a wide literature ranging from 'two by two' games to complex spatial multi-agent (network) models, and from theoretical studies to experimental tests (Binmore et al., 2007; Easley and Kleinberg, 2010b; Cooper et al., 1989; Szabó and Fath, 2007; Perc and Szolnoki, 2010). The coordination problem can be especially difficult when agents do not share the same preferences for different options. These situations are often formalized by asymmetric coordination games, also known as the 'battle of the sexes'. Computational models (Broere et al., 2017; Mazzoli and Sanchez, 2017) and other theoretical studies (Hernández et al., 2017; Hernandez et al., 2013) show that network structure is an important predictor of behavior in iterated asymmetric games. Although these models convincingly show that there are network effects on equilibrium behavior, no empirical studies have been conducted to corroborate these findings. Previous research on network effects on other types of games, such as the Prisoners Dilemma, show that the predictions made by computational models are not always evident when tested empirically (Gracia-Lázaro et al., 2012). It is therefore crucial to test the predictions and assumptions made by these models. In this paper we empirically test predictions made by computational and theoretical studies in an experimental study with human subjects (Broere et al., 2017; Mazzoli and Sanchez, 2017; Hernández et al., 2017; Hernandez et al., 2013).

The problem with asymmetric coordination games is that it is difficult to make behavioral predictions about the outcome of the game. Table 3.1 illustrates a 'battle of the sexes' game in which the two pure-strategy Nash equilibria are: both players play $\alpha$ or both players choose $\beta$. However, the players differ in their preference for the equilibria. Therefore, the game consists of an element of coordination and an element of competition between the players. There is also a mixed Nash equilibrium, but this equilibrium is inefficient, because the expected payoff is lower for both players compared to any of the pure-strategy Nash equilibria. Miscoordination is the most frequent outcome when human subjects play these one-shot games in an experimental setting (Cooper et al., 1989; Binmore et al., 
Table 3.1: Example payoff table asymmetric 'battle of the sexes' game, where the first entry is for player 1 and the second entry for the player 2

\begin{tabular}{lc|cc} 
& Player 2 & $\alpha$ & $\beta$ \\
\cline { 2 - 4 } Player 1 & $\alpha$ & 2,1 & 0,0 \\
$\beta$ & 0,0 & 1,2
\end{tabular}

2007). Both players often choose the behavior according to their own preferred equilibrium, resulting in the lowest payoff for both players. The players often converge to one of the two equilibria when the game is played repeatedly (Duffy et al., 2017; De Kwaadsteniet et al., 2012; Lau and Mui, 2008). In rarer cases players manage to switch simultaneously between the two equilibria, thereby obtaining the best outcome for both players (Duffy et al., 2017).

The situation can be made more complex by adding another player. This means there are three players who have to make a decision between $\alpha$ and $\beta$, but again they differ in their payoff for choosing $\alpha$ or $\beta$. Although the complexity of the situation is increased in terms of the number of players, it is easier to predict the outcome. The payoffs are balanced pairwise between the players, but the global situation is not, because there will always be a majority for one of the options. Intuitively one can already anticipate that when three people have to decide between two options, and two prefer option $\beta$ and only one prefers $\alpha$, the most likely outcome will be $\beta$. The three player situation is illustrated in Table 3.2 , in which the payoffs are the sum of the pairwise interactions of Table 3.1. Again there are two pure-strategy Nash equilibria: all players play $\alpha$ or all players play $\beta$. However, in this case the equilibria are not equivalent and therefore not equally likely. The equilibrium 'all players play $\beta$ ' yields a higher payoff for the two column players. Also, when the column players both play $\beta$, their payoff will at least be two, independent of what player 1 does. When the column players both play $\alpha$, their payoff will be two maximally. No such coordination possibility exists for player 1 and 2 or 1 and 3. Assuming full information for all players, choosing $\beta$ is the best option for both column players. Because the row player is aware of this, the best reply would be to play $\beta$ as well, making 'all players play $\beta$ ' the behavioral prediction in an empirical setting.

The situation changes again when players only interact with a subset of players in the game, instead of all other players. In Figure 3.1 two situations with four players are illustrated. The nodes represent the players and the edges represent which nodes interact with each other. There are two nodes which prefer $\alpha$ and two 
Table 3.2: Example payoff table 3 player asymmetric 'battle of the sexes' game. The first entry is for player 1 , the second entry for player 2 , and the third entry for player 3 .

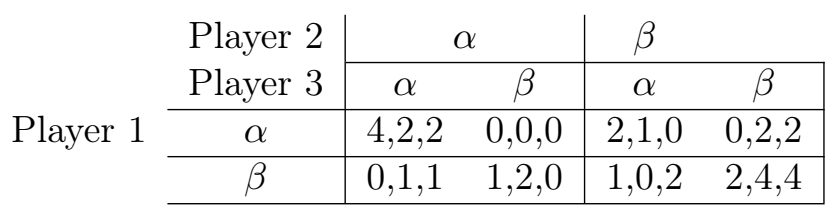

nodes which prefer $\beta$. In the network on the left of Figure 3.1 every node interacts only with nodes who have the opposite preference. Again the equilibria are: all players playing $\alpha$ or all players playing $\beta$. The situation is completely balanced, therefore it is again hard to predict the outcome of the game. The situation illustrated on the left of Figure 3.1 is the same as the situation on the right of Figure 3.1 with the exception that the edge from the top left node to the top right node is replaced with an edge from the top left node to the bottom right node. Although this is a minor change in the interaction structure, it is now easier to predict the outcome of this game. Both players with a preference for $\beta$ always have to interact with players with a different preference. However, both players with a preference for $\alpha$ also interact with each other, making their pairwise interaction no longer an asymmetric battle of the sexes game, but a symmetric coordination game. Therefore, choosing $\alpha$ yields the highest payoff for the pairwise interaction between these two nodes. The node on the top right has no other choice than to coordinate on the same behavior as the node on the bottom right. The node on the bottom left can therefore infer that the other players will choose $\alpha$, making it the best response to also choose $\alpha$. So, 'all players play $\alpha$ ' is the prediction in this situation.

In the 4-player situation one can still reason about what the predictions will be. In situations with more than 4 players it rapidly becomes harder to reason about the behavioral outcome of a game and the situation becomes yet more complicated when there are complex interaction structures added. In these types of situations, computational models are used to develop predictions.

There are several computational studies exploring the behavior of multiple agents with asymmetric 'battle of the sexes' type of dynamics. Several studies explore homogeneous spatial structures such as cellular automata (Alonso-Sanz, 2011; Alonso-Sanz, 2012a; Alonso-Sanz, 2012b). An interesting finding is the ability of self organization in agreement clusters in cellular automata. Clusters of adjacent nodes can coordinate on one behavior while other parts of the cellular 

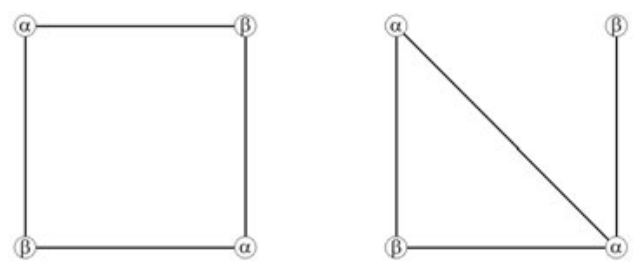

Figure 3.1: Four-player games, represented as a network; $\alpha$ and $\beta$ denote the preferences of the players.

automata can coordinate on the other behavior. Hernández, Muñoz-Herrera and Sánchez introduce a theoretical model for exploring Nash equilibria of battle of the sexes games on Erdös-Rényi networks under the conditions of both complete and incomplete information (Hernandez et al., 2013). They find a rich set of equilibria where both homogeneous and heterogeneous equilibrium behavior is possible when players have complete information. This set is reduced when players have incomplete information. In a subsequent study, the influence of group size and the strength of preferences on equilibrium behavior is studied (Hernández et al., 2017). The stronger the preferences, the harder it is to obtain homogeneous equilibrium behavior. Other related work studies computationally the behavior of dynamic networks in which actors can make or break their links (Bojanowski and Buskens, 2011). This study identifies a set of networks that are stable, in the sense that actors no longer wish to break links or make new ones, often resulting in segregated networks. In addition, some studies consider the influence of heterogeneity in other games such as (weak) Prisoner's Dilemma games (Wang et al., 2014; Szolnoki and Perc, 2014; Amaral et al., 2016). An interesting result is that payoff heterogeneity is predicted to have a favorable effect on cooperation. Furthermore, there are some experimental studies with human subjects testing the effects of network structure on the number of rounds the participants need to reach coordination in both symmetric and asymmetric coordination games. Findings indicate that network structure influences the pace at which participants coordinate and network density can help the coordination process in asymmetry coordination games (McCubbins et al., 2009). Other experimental studies show that with complete information on choices made by all actors in the entire network, participants are quicker in reaching coordination compared to more limited information sets (Enemark et al., 2014). However, these experimental studies do not study the effect 
of network structure on what the equilibrium behavior will be.

All this research, based mostly on computational models, yields specific predictions about global behavior on complex situations. However, obtaining those predictions often requires a lot of simplifying assumptions. The question then arises as to how well do these models predict actual behavior given the assumptions they make and the complexity of the situation? This is the main motivation for the experimental work we report in this paper.

\subsection{Theory}

In this study we empirically test behavioral predictions from previous computational (Broere et al., 2017; Mazzoli and Sanchez, 2017) and theoretical studies (Hernández et al., 2017; Hernandez et al., 2013); specifically, we consider the influence of network structure on equilibrium behavior in iterated asymmetric 'battle of the sexes' games. We focus on the computational model described in Chapter 2. In this study nodes play iterated $2 \times 2$ games with their neighbors. Each node gets a preference assigned before the first round of the game (say $\alpha$ or $\beta$ ), that determines whether the node is a row or a column player as shown in Table 3.2. Every round the nodes choose between $\alpha$ or $\beta$ and the decision of the node is played against all its neighboring nodes. In the first round, the nodes play their preferred behavior with probability one. After every round, the nodes update their probability of playing $\alpha$ or $\beta$ by means of reinforcement learning (Cimini and Sánchez, 2014; Ezaki and Masuda, 2017). The probability of choosing either $\alpha$ or $\beta$ is updated towards what would have been the best choice the previous round. Earlier research investigated the response behavior of human subjects in iterated 'battle of the sexes' games in two types of ring networks (Mäs and Nax, 2016; van Gerwen and Buskens, 2018). This study found that 96 percent of the decisions of the subjects followed a myopic best response pattern. We therefore choose a similar update rule. The update rule is more explicitly described in S1 of the Supplementary Materials. It should be noted that we used a step of 0.5 instead of 0.1 in Equation 2.3 of Chapter 2, because we think this is more realistic for human subjects.

The computational study in Chapter 2 compares the equilibrium behavior in three different types of networks. The baseline model is the Erdös-Rényi (ER) random network. This type of network is usually defined as $G\left(N, p_{e r}\right)$, where $N$ is the number of nodes in the network and $p_{e r}$ the probability of drawing an edge between 
two arbitrary nodes. Thus, for every two nodes in the network the probability that an edge is present is the same. Depending on the choice of the parameters, this usually leads to a network with low clustering and relatively low variation in the degree distribution. The computational study predicts that equilibrium behavior is often homogeneous in these networks, in the sense that all nodes end up choosing the same behavior, all $\alpha$ or all $\beta$. However, it is hard to predict which equilibrium the nodes will end up playing, mostly because this is dependent on a complex combination of network characteristics. Both equilibria (all $\alpha$ or all $\beta$ ) are generally equally likely. This situation closely resembles the situations described in Table 3.1 and the left network of Figure 3.1.

The second type of network studied in the computational study are clustered networks. The clustered networks are obtained by means of the Watts-Strogatz algorithm (Watts and Strogatz, 1998). The algorithm starts with a lattice consisting of $N$ nodes. Each node is connected with $n$ neighboring nodes. The edges are rewired randomly with a constant probability for all edges. Using relatively low values of the rewiring probability, the characteristic of these networks are short average path lengths and high clustering, known as the 'small world' network characteristics. The computational study predicts that equilibrium behavior is homogeneous within the clusters, but heterogeneous between clusters, resulting in heterogeneous behavior in the overall network. Similar effects are shown in an experiment with different (learning) dynamics (Vriens and Corten, 2018). This result is pretty intuitive when taking a closer look at the definition of clustering. Clustering is often defined by the ratio of edges within a cluster and the edges outside the cluster (Fortunato, 2010; Newman, 2006). The higher this average ratio, the higher the clustering of the network. In clustered networks coordinating on the same behavior within the cluster is simply more rewarding because there are on average more edges within the community than outside. This causes different communities to coordinate on different choices. A good predictor of the behavior within the cluster is the preference of high degree nodes. The higher the degree of a node, the higher the probability that the node converges to its preferred behavior.

The third type of network studied in the computational study are centralized networks. This type of network is constructed using the preferential attachment algorithm (Barabási and Albert, 1999). This algorithm starts with one or more nodes and new nodes are added iteratively. In each iteration, the probability for a new node to connect to an existing node depends on the number of links the existing nodes already have; the more links a node already has, the higher the 
Table 3.3: Number of neighboring nodes required for a local majority given the degree of a node. Nodes with an even degree always need $50 \%$, while an uneven degree requires more than $50 \%$ of its neighbors.

\begin{tabular}{l|cccccccc}
\hline Degree centrality & 1 & 2 & 3 & 4 & 5 & 6 & 7 & 8 \\
Number of neighboring nodes needed & 1 & 1 & 2 & 2 & 3 & 3 & 4 & 4 \\
Percentage of neighboring nodes needed & $100 \%$ & $50 \%$ & $67 \%$ & $50 \%$ & $60 \%$ & $50 \%$ & $57 \%$ & $50 \%$ \\
\hline
\end{tabular}

probability that the new node gets connected to it. This creates a 'rich get richer' effect. In the computational study, the centralized networks had low-clustering, a few central nodes with high degree centrality and a majority of peripheral nodes with low degree centrality. The equilibrium behavior in these networks is, just like random networks, often homogeneous in the sense that all nodes end up choosing the same option: all $\alpha$ or all $\beta$. However, which equilibrium the nodes converge to is easily predicted by the preference of the nodes with high degree centrality. In these networks the nodes with the highest degree centrality could dictate the equilibrium behavior of the overall network. This situation closely resembles the situation of the right network in Figure 3.1.

Another effect which is present in all types of networks is related to an even degree centrality. Nodes with an even degree centrality can relatively easily coordinate on their preferred behavior compared to nodes with a similar but uneven degree centrality. This situation can be understood by reviewing the 3 player situation described in Table 3.2. When nodes have an even degree, the total number of relevant choices is uneven, including the node itself. So locally there is always a majority for one of the choices as described earlier in Table 3.2. Table 3.3 exemplifies the situation up to degree centrality eight. The percentage of neighboring nodes that have to choose the same behavior for a local majority is always 50 percent for nodes with an even degree centrality, while it requires more than 50 percent for nodes with an uneven degree centrality. This effect was also found in symmetric coordination games in a computational study by Buskens and Snijders (2005).

Summarizing, computational studies predict homogeneous equilibrium behavior in random networks, while the predicted probability is often close to 50 percent for either all $\alpha$ or all $\beta$. So, the information which can be derived from the network is limited. The predicted equilibrium behavior in centralized networks is also homogeneous, but the equilibrium behavior can be predicted by a few nodes with high degree centrality. In clustered networks degree centrality is also a good predictor of behavior, but behavior is restricted to the community the node belongs 
An experimental study of network effects on coordination in asymmetric games

to. Finally, nodes with an even degree centrality more easily coordinate on their preferred behavior than nodes with an uneven degree centrality. Based on these computational predictions, we will empirically test the following hypotheses:

\section{Hypothesis 1:}

The correlation of (equilibrium) behavior between the computational model and the empirical results will be higher for centralized networks and clustered networks compared to random networks.

\section{Hypothesis 2:}

The equilibrium behavior is more heterogeneous in clustered networks compared to random and centralized networks.

\section{Hypothesis 3:}

Participants with high degree centrality will more often play their preferred behavior.

\section{Hypothesis 4:}

Nodes with an even degree centrality more often play their preferred behavior than nodes with uneven degree centrality in all networks.

\subsection{Methods}

\subsubsection{Experimental setup}

In order to test the four hypotheses we carried out a computerized laboratory experiment on networks of size 20. We used the Python based software platform oTree (Chen et al., 2016). The experiments where conducted both at Universidad Carlos III de Madrid, Spain and Utrecht University, the Netherlands. In total 140 subjects participated in Madrid between April 9th and April 20th 2018. These participants were invited from the IBSEN volunteer pool (IBSEN, 2018). In addition, 100 subjects participated between May 22nd and May 30th in the Experimental Laboratory for Sociology and Economics (ELSE), Utrecht. These participants were invited using the ORSEE recruitment system (Greiner et al., 2004). In total we conducted 12 sessions with 20 participants. Therefore $\mathrm{N}=240$ 
Table 3.4: Payoff table for the experiment

\begin{tabular}{cc|cc} 
& Player 2 & Blue & Yellow \\
\cline { 2 - 4 } Player 1 & Blue & 10,8 & 0,0 \\
& Yellow & 0,0 & 8,10
\end{tabular}

in total.

During the experiment, participants played an iterated asymmetric 'battle of the sexes' game against multiple other participants at the same time. Every participant interacted with a subset of other players. Interactions are mapped on a network in which the players are nodes and they play the game with the participants to whom they are connected. The payoff table is shown in Table 3.4. The payoffs are chosen such that the difference between coordinating on the preferred option and the not preferred option is a real difference, but small enough for participants to have incentive to deviate from their own preference. Participants can choose between 'blue' and 'yellow' and play the same choice against all participants they interact with.

In this study we used three different networks, each to represent the characteristics of random networks, clustered networks and centralized networks. The networks used in the experiments are represented in Figure 3.2 and the adjacency matrices can be found in Table A6 till Table A8 of the appendix. The network on the left in Figure 3.2 has low clustering and low differentiation in degree centrality of the nodes. The network in the center of Figure 3.2 has high clustering and low differentiation in degree centrality of the nodes. The network on the right in Figure 3.2 has low clustering and a few nodes with high degree centrality. All networks have a network size of 20 nodes and a network density of 0.2 . The network size is big enough that it contains non-trivial complexity and small enough to make it experimentally feasible. The computational study shows that the hypothesized outcomes can be expected for networks of size 20 and in a similar way for larger network sizes (Broere et al., 2017).

Upon arrival participants were seated randomly in the laboratory. All subjects participated in games on all three networks. Before the first round of each network, participants were assigned their 'type' at random. The type determined whether they were the row or the column player in Table 3.4. The randomization was performed with the constraint of 10 row players and 10 column players to maximize the coordination problem. In every network, the participants played 20 rounds in 

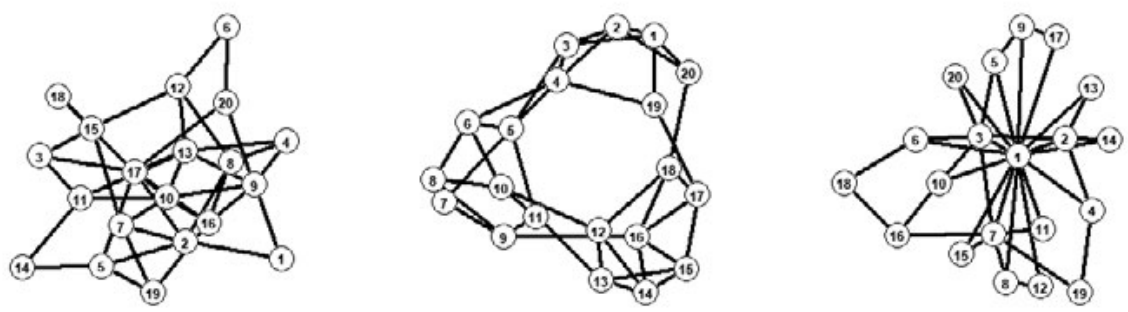

Figure 3.2: 20 -player games, represented as a network. Left the random network, in the middle the clustered network, on the right the centralized network.

which their type, location in the network, and the participants they interacted with are the same. The order in which participants were placed on each one of the networks was varied between sessions.

In each round participants had to decide between 'blue' and 'yellow'. After all participants had made their decisions, they where all informed about their payoff in that round and how many of the participants he/she is connected with played 'blue' or 'yellow'. The participant did not receive information about players in the network he or she was not connected with. Next, the participants were asked to make a new decision for the next round, continuing for 20 rounds. We informed the participants beforehand that they would play 20 rounds. We communicated the exact number of rounds because we did not expect any end game effects. Before the actual rounds were played the participants were asked to read the instructions of the game. After that, they were asked to answer questions about the instructions in order to test whether they understood the game. Before the participants played on the actual networks, three practice rounds on a random network were played.

The experiments lasted an average of 45 minutes. The payoff of each round was accumulated and then divided by the number of opponents (interaction partners in the network). For every 50 points earned, the participants received 1 euro. In addition, they received 5 euros show up fee. The maximum that could be obtained was 17 euros. The average payoff was around 14 euros, the lowest was 10.50 euros, the highest 16.50 euros. After the experiment, participants were asked to leave the room, after which they were invited one by one to collect their 
earnings in privacy. The experiments in Spain were in Spanish and the experiments in the Netherlands were in English. The instructions in both languages can be found in Figure A8 and Figure A9 of the appendix. The oTree code together with the obtained data and the R-scripts for the analyses can be found on the first authors github page: https://github.com/JJBroere/An-experimental-studyof-network-effects-on-coordination-in-asymmetric-games.

\subsubsection{Measures and analytical strategy}

In order to test hypothesis 1 , we have to calculate the correlation between the computational model and the empirical results. The correlations are computed with the same starting conditions; the same network and preferences of the actors in the same positions on the network. Given the same starting conditions, how often do the nodes in the computational model make the same choice (blue or yellow) as the people in the experiment? Thus, the correlation of the behavior between the computational graph $g^{c}$ and the behavior of the empirical graph $g^{e}$ for each graph is defined as:

$$
r=\operatorname{cor}\left(g^{c}, g^{e}\right)=\frac{\sum_{i=1}^{N} \sum_{t=1}^{R} f\left(x_{i t}^{c}, x_{i t}^{e}\right)}{N \times R},
$$

where $x_{i t}$ is the behavior ('blue' or 'yellow') of node $i$ at round $t, N$ is the number of nodes and $R$ the number of rounds. The function $f\left(x_{i t}^{c}, x_{i t}^{e}\right)$ can have two values,

$$
f\left(x_{i t}^{c}, x_{i t}^{e}\right)= \begin{cases}1 & \text { for } x_{i t}^{c}=x_{i t}^{e} \\ -1 & \text { for } x_{i t}^{c} \neq x_{i t}^{e} .\end{cases}
$$

The computational model is run with the same initial conditions (distribution of types) as the empirical result. Because the computational model has a stochastic update rule, we run the model 100 times and report the median correlation between the model and the empirical result. We chose to report the median, because in some cases the distribution can be very skewed. For the full specification of the computational model see appendix and Chapter 2.

In order to test hypothesis 2 we resort to an analysis of variance (ANOVA) with post hoc tests on the heterogeneity of behavior in the networks in the last 5 rounds. We chose to look at the last 5 rounds instead of just the last round because random switching of behavior in the last rounds can influence the results. By taking the average of the last five rounds, the effects of switching or possible end game effects 
An experimental study of network effects on coordination in asymmetric games

Table 3.5: Correlation between the computational model and the empirical results. The correlation is defined in the text. The percentage correct is the percentage correctly predicted behavior of the experimental results by the computational model

\begin{tabular}{l|cccc}
\hline & \multicolumn{2}{|c}{ All rounds } & \multicolumn{2}{c}{ Last five rounds } \\
& Correlation (sd) & \% correct (sd) & Correlation (sd) & \% correct (sd) \\
\hline \hline Random & $0.13(0.09)$ & $56(4.61)$ & $0.18(0.16)$ & $59(8.29)$ \\
Clustered & $0.44(0.28)$ & $72(6.46)$ & $0.51(0.13)$ & $76(6.65)$ \\
Centralized & $0.74(0.17)$ & $87(8.74)$ & $0.92(0.25)$ & $96(12.48)$ \\
\hline $\mathrm{N}$ & 12 & 12 & 12 & 12 \\
\hline
\end{tabular}

will be minimized in the analyses. We define heterogeneity of behavior as the variance of participants choosing 'blue' in the network:

$$
h\left(p_{\text {blue }}\right)=\operatorname{var}\left(p_{\text {blue }}\right)=p_{\text {blue }}\left(1-p_{\text {blue }}\right),
$$

where $p_{\text {blue }}$ is the proportion of nodes playing 'blue' in the last 5 rounds of the game.

We test hypotheses 3 and 4 by means of a multilevel logistic regression for all subject decisions over all 20 rounds. Three levels are specified, taking into account the repeated measures of the participants and the correlation within networks. The dependent variable is 1 when the participant plays its preferred behavior and 0 otherwise. The predictor variables are the degree centrality of the participant in the network and the evenness of the degree.

\section{Results}

In Table 3.5, the correlations between the empirical results and the computational model for each network are presented. We investigate how well the computational model predicts both the empirical behavior in all 20 rounds and the convergence behavior of the last five rounds. It is hard to define an objective convergence criterion, because participants sometimes switch behavior in an equilibrium state, presumably trying to persuade their neighbors to switch as well. We therefore apply a more subjective interpretation of convergence using as rule of thumb whether or not the switching of a player leads to switching behavior for other players as well. According to this interpretation, convergence is reached in the last five rounds in all sessions for the clustered network in Figure 3.2, and for the network with the central node in Figure 3.2. Not all sessions converged in the random network in Figure 3.2. Six out of twelve networks did not fully converge in the last five 
rounds. This can occur because the switching of behavior was still effective, inducing neighbors switching behavior as well, while in the other two networks it rarely happened that switching behavior led to neighbors switching behavior as well. See Figure A10 till Figure A15 of the appendix for a visual example of the behavior of the computational model compared to the experimental results.

The correlation between the empirical results and the computational model is $r=0.13, s d=0.09$ for random networks over all 20 rounds and $r=0.18, s d=0.16$ for the last five rounds. The random network closely resembles the original two player game in Table 3.1 or the situation on the left in Figure 3.1. In these situations different equilibria are equally likely. This is also reflected in the current results where the computational model poorly predicts the empirical behavior. The correlation of the clustered network is $r=0.44, s d=0.28$ and $r=0.51, s d=0.13$ for the last five rounds. This means that the model predicts 76 percent of the empirical observations correctly in the last five rounds. Predictions for these types of networks are significantly better compared to the random network. Finally, for the centralized network, the median correlation is $r=0.74, s d=0.17$ in all rounds and $r=0.92, s d=0.25$ for the last five rounds, meaning that towards the end behavior is predicted correct in almost all cases: in fact, out of 12 sessions the behavior was predicted wrongly only twice. In these cases the empirical behavior converged to the complete opposite direction from the predicted behavior, leading to a correlation of -0.98 and -1 . This is reflected in the standard error. However, 10 out of 12 times the empirical results are correctly predicted by the computational model. Based on these results we found evidence in favor of hypothesis 1 .

In order to test hypothesis 2 , we examine heterogeneity of behavior in the last five rounds of the games for the different networks. The mean variance in the random networks is, $\mu=0.116, s d=0.099$, although six out of twelve networks have not converged. The mean variance in the clustered networks is, $\mu=0.183, s d=0.085$, corresponding to a situation in which clustered networks converged most of the time to a state where around half of the participants choose 'blue' and the other participants choose 'yellow'. The mean variance in the networks with a central node is, $\mu=0.024, s d=0.030$. This indicates that these networks converge to homogeneous behavior where all participants choose 'blue' or all participants choose 'yellow' every time. An ANOVA was conducted to compare the effect of the networks on the heterogeneity in the last five rounds. The ANOVA test yielded significant variation among conditions, $F(2,33)=12.6, p<0.001$. A post hoc Tukey test further shows that the random networks and the clustered networks do 
An experimental study of network effects on coordination in asymmetric games

Table 3.6: Multilevel logistic regression results, dependent variable is 1 if the participant chose his or her preferred behavior, 0 otherwise. Three levels are specified, taking into account the repeated measures of the participants and the correlation within networks.

\begin{tabular}{lcccc}
\hline \hline & All Networks & Random & Clustered & Centralized \\
\hline Degree centrality & $0.118^{* * *}(0.013)$ & $0.185(0.138)$ & $0.280(0.401)$ & $0.210^{* *}(0.085)$ \\
Even degree & $0.164^{* * *}(0.060)$ & $-0.305(0.473)$ & $1.033^{* *}(0.476)$ & $-0.400(0.500)$ \\
Constant & $0.228^{*}(0.136)$ & $1.171^{*}(0.638)$ & $-0.155(1.638)$ & $0.922^{*}(0.493)$ \\
& & & & \\
Random & & & & \\
Subject & $1.913(1.383)$ & $9.231(3.038)$ & $9.635(3.104)$ & $12.13(3.483)$ \\
Network & $0.017(0.132)$ & & & \\
\hline Observations & 14,400 & 4,800 & 4,800 & 4,800 \\
logLik & -7956.3 & -1846.1 & -1767.9 & -1712.8 \\
\hline \hline Note: & & & ${ }^{*} p<0.1 ;{ }^{* *} p<0.05 ;{ }^{* * *} p<0.01$
\end{tabular}

not differ significantly at $p=0.101$, although it should again be noted that the random network often did not converge. The clustered networks and the networks with a central node differ significantly at $p<0.001$; the random network and the centralized network differ significantly at $p=0.019$. Although the difference between the clustered network and the random network is not evident, we do believe that the random networks that did not converge yet would converge to the homogeneous state if more rounds where played. The random networks that did converge, all converged to a (mostly) homogeneous state. Furthermore, we found a very clear difference between the random network and the centralized network in terms of heterogeneity of behavior. We therefore conclude that we have empirical evidence in favor of hypothesis 2 .

In Table 3.6 the results of the multilevel logistic regression analyses are presented. The dependent variable is a dichotomous variable indicating whether a participant chose the preferred behavior. The regression is conducted for all networks together, and with the three networks separately. In the model with all networks together there is a positive statistical significant effect of degree centrality on playing ones preferred behavior, $\beta=0.118, s d=0.013, p<0.001$. The higher the degree centrality, the higher the probability that the participants will play their preferred behavior. There is also a positive statistically significant effect of an even degree centrality, $\beta=0.164, s d=0.060, p=0.006$. If a participant has an even degree centrality, the probability that the participant will play his 
or her preferred behavior is higher as well. Looking at the random networks, there is no statistically significant effect of any of the predictors. In the clustered network there is a statistically significant effect of an even degree centrality, $\beta=1,033, s d=0.476, p=0.029$. There is no statistical effect of degree centrality. In the centralized network we do find a statistical significant effect of degree centrality, $\beta=0.210, s d=0.085, p=0.013$. However no statistically significant effect of having an even degree centrality. We found evidence in favor of hypothesis 3 and hypothesis 4 when combining all the data. However, the hypotheses could not be confirmed in the networks separately. This could be caused by a lack of statistical power and variation in the degree distribution within some types of networks.

\subsection{Conclusion and discussion}

In this paper we empirically studied the effects of network structure on behavior in iterated 'battle of the sexes' games with human subjects. The hypotheses were derived from computational models from previous studies. The empirical results convincingly show that the computational models have empirical validity. The correlations between the computational model and the empirical behavior indicates that network structure governs the behavior to some extent. As expected, as the network structure becomes less 'random', the more accurate the computational model prediction of the behavior of the players. Furthermore, we found evidence that the major network effects found in the computational study have a clear counterpart on the behavior of the game when played with human subjects. Thus, in the clustered networks the equilibrium behavior was clearly more heterogeneous compared to the centralized networks. In random networks we did not find a significant difference with the clustered network. However, this could partially be explained because half of the sessions with the random networks did not converge after 20 rounds and more homogeneity could still be expected. We also found evidence that degree centrality and having an even degree plays a role in determining the behavior of the game.

Although the experiments were conducted in relatively small and simple networks, we do believe that the results are generalizable to bigger and more complex network settings. As both the computational and the empirical results show, most of the behavior is limited to the clusters of the network, and within each cluster our reasoning in terms of how majority influences decision presented above seems to apply quite well. When a larger network consists of multiple clusters, the same behavior can be expected within the clusters of the larger network. In larger 
networks with low clustering the same dynamics can be expected as in smaller networks, however the relative influence of a single node on the equilibrium behavior naturally becomes smaller as the number of nodes in the network becomes larger.

The small and limited number of networks studied is however a limitation of the current study. In this study we chose three fixed networks to represent networks used in the computational study; namely an Erdös-Rényi random network, a small world network and a preferential attachment network. There are several caveats to make about these choices. First of all, the networks belong to a class of network defined by their mathematical properties. We did not study any variation between networks within this class. The lack of variation within one network also makes it harder to detect the influence of different nodes. Secondly, the mathematical properties of the networks are often limiting properties as the number of nodes goes to infinity: although the effects are independent of size in the computational study, the networks in this study might in fact be too small to represent the limiting properties of the class of networks. However, studying larger and/or more different networks is complicated and expensive in an experimental context with human subjects, because this would require very large sample sizes and also a large amount of participant fee. The limiting factor is in the between network comparisons. In the current study it required 240 participants to be able to compare 12 networks per type.

All in all, our experiments show that the behavior of people trying to coordinate in a network can be understood reasonably well in terms of local considerations. In other words, subjects react to what they observe around them, the rest of the network does not seem to be relevant. This opens an interesting avenue of research as in other situations, different from the one considered here, global information may be available that changes how people behave. Indeed, it was observed that when the total number of people making one or the other choice is made available to the subjects, global coordination is reached much more easily in a population of moving subjects(Antonioni et al., 2014). In the case of networks, knowing that there are very many players choosing the option one prefers may help to insist on making the decision that goes in one's interest, in the hope that eventually one's neighbors might conform to the global majority. This, along with trying to extend our experiments to larger networks and to more representatives of each class, would be an interesting contribution to establish the knowledge on coordination on networks on firm grounds. 



\title{
Chapter 4
}

\section{Asymmetric games on networks and the Ising model ${ }^{1}$}

\begin{abstract}
Many computational and theoretical studies show that network structure can have an important effect on the evolution of behavior in iterated $2 \times 2$ games. However, the results of these models often depend on simplifying assumptions, such as the update rule. Understanding the role of simplifying assumptions is therefore necessary for the interpretation of the outcomes and the generalizability of these models. We study the influence of such simplifying assumptions for an iterated asymmetric 'battle of the sexes' game by comparing it to an implementation using the ferromagnetic Ising model. In order to represent the conflicting interests of players in a 'battle of the sexes' game, we further generalize the Ising model by allowing the 'external magnetic field' parameter to vary between actors. We find a richer and more detailed set of equilibria by modeling the game as an Ising model. Which theoretical model yields the best predictions for empirical situations is dependent on the studied empirical situation at hand.
\end{abstract}

\footnotetext{
${ }^{1}$ This chapter is based on a paper written by Joris Broere, Vincent Buskens and Henk Stoof. Broere wrote the manuscript and conducted the analyses. Buskens contributed substantially to the manuscript. Stoof initiated the project and contributed substantially to the manuscript.
} 


\subsection{Introduction}

Social dilemmas occur when individual rational behavior leads to an outcome that is less desirable compared to situations in which the actors had cooperated (Raub et al., 2015; Buskens and Raub, 2013). In these situations, actors can benefit by uncooperative choices, unless the other actor(s) choose uncooperative as well (Van Lange et al., 2013). Game theory is often used to formalize these types of situations (Binmore, 1994). Examples of social dilemmas in game theory are the prisoner's dilemma and some types of asymmetric coordination games. Understanding when cooperation or coordination occurs is fundamental to understanding behavior in real world situations, such as free riding behavior, overexploitation of common goods and conflict in general (Allison and Kerr, 1994; Hardin, 1968; Broere et al., 2017). This has led to a large body of work trying to understand when cooperation occurs and when it breaks down (Raub et al., 2015; Macy and Flache, 2002). Repeated interactions and network structure are often suggested as possible solutions for different variations of social dilemmas (Axelrod and Hamilton, 1981; Grujić et al., 2010; Nowak and May, 1993; Perc et al., 2013; Perc and Szolnoki, 2010; Perc and Szolnoki, 2008). However, the results often depend on simplifying assumptions of the models, such as the update rule (Gracia-Lázaro et al., 2012; Roca et al., 2009). Therefore, the simplifying assumptions are important to study in order to understand the validity and generalizability of these models. In this study, different ways of formalizing of one such social dilemma can affect the predicted outcome of the game. We hereby focus on the asymmetric coordination game known as 'battle of the sexes'.

The effect of networks in iterated game is a rigorously studied subject. There is substantial literature on theoretical, computational and experimental studies for different games (Binmore et al., 2007; Easley and Kleinberg, 2010b; Cooper et al., 1989; Szabó and Fath, 2007; Perc and Szolnoki, 2010; Broere et al., 2017; GraciaLázaro et al., 2012; Broere et al., 2019). Many computational studies use repeated decisions in discrete time steps that lead to some form of convergence. This way of formalizing network games requires a large set of assumptions. In iterated games, all players make decisions simultaneously at fixed time points. The decision for the next time point is updated by a fixed update rule in which information of other players choices is partly revealed. By making repeated decisions, a correlation is introduced into the behavior of the players, as the decision of a player at time $t$ is dependent on the various decisions made at $t-1$. Because the correlation determines how much the choice made in time point $t$ is dependent on $t-1$, the 
strength of this correlation might be an important feature to study.

In discrete time models the correlation between decision time points might vary between different update rules, but also in how the update rule is specified. For instance, the correlation can be influenced by introducing a random error term into the update rule. The bigger the error term, the less $t$ depends on $t-1$. However, even though the error term in an update rule might influence the correlation between time points, it cannot be used to model the correlation between time points explicitly. Studying the effect of the correlation explicitly can lead to valuable information, because it represents the importance of previous time points or the strength of interaction in general.

We here study the effect of the update rule by modeling the same situation as a repeated interaction model and with the ferromagnetic Ising model. The ferromagnetic Ising model studies alignment of dichotomous variables that represent positive and negative atom spins. We will parametrize the ferromagnetic Ising model such that the alignment will represent the coordination problem of the battle of the sexes. In the Ising model variables interact with a external magnetic field. We allow the magnetic field to take on different and opposite signs for different nodes in the network in order to represent the different preferences in the battle of the sexes. The Ising model also allows for the modeling of the correlation explicitly by means of an interaction coefficient between the Ising spins. The Ising model can provide analytical solutions for discrete variables on simple networks and does not depend on an update rule. We will compare analytic expectations of ring networks with results from previous computational studies and draw conclusions about the validity of both methods (Broere et al., 2017; Mazzoli and Sanchez, 2017; Hernández et al., 2017; Hernandez et al., 2013).

\subsubsection{The battle of the sexes as a spin system}

Coordination is fundamental to any form of organization. Some types of coordination are just a matter of convention, such as on which side of the road people drive (Voss, 2001). This type of coordination is unproblematic in the sense that it does not matter from person to person on which side of the road they drive, as long as everybody chooses the same side. Coordination can become more difficult when people prefer different options. For instance, when a group of people work on a project together and some people would like to use software $X$ for the project, while others have a preference for software $Y$. In this case, coordination can lead to frustration as some people will have there preference fulfilled, while 
others do not (Hernández et al., 2017; Hernandez et al., 2013). When an actor is coordinating on an outcome that is not favorable for the actor itself, the actor shows cooperative behavior while at the same time the actor can be frustrated about not meeting its preferred behavior.

Situations of coordination where the agents have different preferences are often formalized as an asymmetric coordination game known as 'the battle of the sexes'. The battle of the sexes is an interesting game because it contains both the element of coordination and the element of competition (Binmore et al., 2007). In the example game described in Table 4.1a, the players have to choose between two options, in this case $\alpha$ or $\beta$. The numbers indicate the payoffs the players obtain given their own choice and the choice of the other player. Coordinating on the same choice yields a higher payoff for both players compared to miscoordination. The pure Nash equilibria for this game are, both players choose $\alpha$ or both players choose $\beta$. However, the players differ on which of the equilibrium they prefer. Player 1 has a higher payoff when both players choose $\alpha$ and player 2 has a higher payoff when both players choose $\beta$. The mixed strategy solution of this game is suboptimal, since it yields a lower expected value for both players compared to both pure strategy equilibria.

Experimental studies where human subjects play one-shot battle of the sexes games tend to corroborate the suboptimal mixed strategy solution. In an experimental study, miscoordination happens 52 percent of the time (Cooper et al., 1989). In that study, the payoff matrix is defined as $S_{1}=S_{2}=1 / 2$ in Table 4.1a. This finding is very close to the mixed strategy solution, which predicts miss-coordination to happen $5 / 9$ percent of the time and coordination $4 / 9$ percent of the time. A solution to overcome this suboptimal outcome is by playing the game repeatedly amongst the same two players. The players can learn from past choices of the other player and adjust their choice based on the rounds before. The repeated play creates a correlation in the choices of the players. By knowing past behavior, players can anticipate future behavior. Experimental studies with human subjects who play the game repeatedly against the same player almost always manage to coordinate after a couple of rounds (Duffy et al., 2017; De Kwaadsteniet et al., 2012; Lau and Mui, 2008).

The Ising model can represent the binary choice options of the battle of the sexes while taking into account correlations in choices. The Ising model is a classic model from the field of statistical physics (Ising, 1925; Glauber, 1963). It models binary variables that interact pairwise given a graph structure. In the original 
Table 4.1: Left: Example payoff table asymmetric 'battle of the sexes' game, where the first entry is for player 1 and the second entry for player 2 , where $0<S_{i}<1$ with $i \in 1,2$. Right: example of two interacting spins, where the energy-levels $E$ are defined by two magnetic fields $B_{1}$, for player 1 and $B_{2}$ for player 2 , and an interaction coefficient $J$, where $J>0, B_{1}>0$ and $B_{2}<0$, for an energy landscape that models the same dilemma as the battle of the sexes game.

\begin{tabular}{|c|c|c|c|c|c|c|}
\hline & Player 2 & $\alpha$ & $\beta$ & & $\uparrow$ & $\downarrow$ \\
\hline Pla & $\alpha$ & $1, S_{1}$ & 0,0 & $\uparrow$ & $-J-B_{1}-B_{2}$ & $J-B_{1}+B_{2}$ \\
\hline
\end{tabular}

(a)

(b)

application it models the alignment of atomic spins that can be in a positive or negative spin state. The Ising model is later used to model many different systems with interacting binary variables, with application in fields like sociology, psychology, neuroscience and game theory (Hopfield, 1982; Borsboom and Cramer, 2013; Haslbeck et al., 2018; Hauert and Szabó, 2005). In this paper we use the Ising model to model the binary choice options of actors in an asymmetric coordination game who interact given a network structure.

The game in Table 4.1a can be written as the energy landscape of two interacting atomic spins, as stated in Table $4.1 \mathrm{~b}$. Table $4.1 \mathrm{~b}$ represents the energy landscape of two atoms that can be in one of two states, namely up or down, where the energy function of the configuration is defined as $E(s)$. The probability of an atom of being in the up state or the down state is dependent on an external magnetic field $B$ and the interaction coefficient $J$. The field $B$ determines the attraction of (or preference for) the atom to be in one of the spin states. Important to note is that the external magnetic field can be different for the two players. External magnetic field $B_{1}$ interacts with player 1 and external magnetic field $B_{2}$ interacts with player 2. The interaction coefficient $J$ determines the correlation of behavior between the two atoms. The model is called ferromagnetic when $J>0$, meaning that the atoms have a higher probability for alignment ( $\uparrow$ or $\downarrow \downarrow)$. The model is called anti-ferromagnetic when $J<0$, meaning that the atoms have a higher probability for the opposite direction ( $\uparrow \downarrow$ or $\downarrow \uparrow$ ). The model is called non-interactive when $J=0$. The coupling constant $J$ can be interpreted as the correlation of behavior between the two atoms, so when $J=0$ the energy landscape is equal to the one-shot situation in Table 4.1a with a mixed equilibrium that is uncorrelated.

In order to see how Table 4.1a and Table 4.1b are related, let us look at an 
example. The probability of the particles to be in one of the four states of the energy landscape is

$$
P\left(s_{1}, s_{2}\right)=\frac{e^{-E(s)}}{Z},
$$

where $Z$ is a normalizing constant defined as the sum of $e^{-E(s)}$ over all possible configurations, $s_{1}$ is the state of atom 1 (or player 1 ), which can be up or down, $s_{2}$ is the state of atom 2 (or player 2), which can also be up or down, and $E$ is the energy of the combined state $s$, determined by the parameters $J, B_{1}$ and $B_{2}$. In the uncorrelated case where $J=0$, the joined state is equal to the multiplication of the probabilities that the individual spins are in that state, $P\left(s_{1}, s_{2}\right)=P_{1}\left(s_{1}\right) P_{2}\left(s_{2}\right)$. The probability of states of the uncorrelated landscape in Table $4.1 \mathrm{~b}$ is equal to the probabilities of the uncorrelated (one-shot) mixed strategy in Table 4.1a if the parameters relate in the following manner

$$
B_{1}=\ln \sqrt{\frac{1}{S_{1}}},
$$

and

$$
B_{2}=-\ln \sqrt{\frac{1}{S_{2}}} .
$$

For example, lets define the game in Table 4.1a as $S_{1}=S_{2}=1 / 2$. The mixed strategy solution in this situation is for player 1 to play $\alpha$ two thirds of the time and $\beta$ one third of the time and for player 2 to play $\beta$ two thirds of the time and $\alpha$ one thirds of the time. Therefore, the probabilities of the different strategy combinations are $2 / 9$ for $(\alpha, \alpha), 4 / 9$ for $(\alpha, \beta), 1 / 9$ for $(\beta, \alpha)$ and $2 / 9$ for $(\beta, \beta)$. The probability of being in the $(\uparrow \uparrow)$ state can now be calculated by filling in Equation 4.1,

$$
P(\uparrow \uparrow)=\frac{e^{-\left(-J-B_{1}-B_{2}\right)}}{e^{-\left(-J-B_{1}-B_{2}\right)}+e^{-\left(J-B_{1}+B_{2}\right)}+e^{-\left(J+B_{1}-B_{2}\right)}+e^{-\left(-J+B_{1}+B_{2}\right)}}=2 / 9
$$

where, $J=0$ and $B_{1}=-B_{2}=\ln \sqrt{2}$. Filling in Equation 4.1 for the other states yields $P(\uparrow \downarrow)=4 / 9, P(\downarrow \uparrow)=1 / 9$ and $P(\downarrow \downarrow)=2 / 9$, as designed.

In the next sections, we use a computational method and the Ising model to study the equilibrium behavior for small networks. We use the Ising model to calculate analytical results for these networks, where we vary the interaction coefficient $J$ in order to represent the repeated interactions of the network games. Due to the nature of the battle of the sexes game the interaction coefficient is ferromagnetic, as the player prefer to coordinate their behavior. We compare the results of both methods. 


\subsubsection{The battle of the sexes on a network}

In recent studies, the effect of spatial structures on the equilibrium behavior of asymmetric coordination games became of interest. Several studies explore homogeneous spatial structures such as cellular automata (Alonso-Sanz, 2011; AlonsoSanz, 2012a; Alonso-Sanz, 2012b). An interesting finding is the ability of self organization in agreement clusters in cellular automata. Clusters of adjacent nodes can coordinate on one behavior while other parts of the cellular automata can coordinate on the other behavior. Hernández, Muñoz-Herrera and Sánchez (2013) introduce a theoretical model for exploring Nash equilibria of battle of the sexes games on Erdös-Rényi networks under the conditions of both complete and incomplete information. In the complete information case, the actors know what type of actors the other players in the network are. In the incomplete information case, the players only have believes about the other player represented by a probability distribution. They find a rich set of equilibria where both homogeneous and heterogeneous equilibrium behavior is possible when players have complete information. This set is reduced when players have incomplete information. In a subsequent study, the influence of group size and the strength of preferences on equilibrium behavior are studied (Hernández et al., 2017). Computational models show that network structure can be an important predictor of behavior for iterated battle of the sexes games (Broere et al., 2017). This study compares the convergence behavior in preferential attachment, small world and Erdös-Rényi networks. The findings suggest that clustering in networks facilitates self organization of uniform behavior within clusters, but the clusters themselves behave more or less independent from the rest of the network. Furthermore, nodes with a higher degree centrality and nodes having an even degree are more likely to converge to their preferred equilibrium and can therefore be used to predict the behavior in the full network. In a subsequent study, predictions made by the computational study were validated in an experimental study with 240 human subjects (Broere et al., 2019).

There are many examples of studies that utilize the Ising model to study game theoretical situations (Galam and Walliser, 2010; Stauffer, 2008; Perc et al., 2017). Different game have been studied. One study implements an asymmetric Ising model to represent cooperation in a public goods game (Bruggeman et al., 2018). They show that a critical level of noise can trigger a cascade of cooperation. Another paper uses the Ising model to study Nash equilibria in the response strategy of 'correlated' Snowdrift games (Correia and Stoof, 2019). They show that in game 
theoretical situations that are naturally correlated, there is a wide set of Nash equilibria possible when the players are allowed to deviate from the correlation. They find these solutions to be describable by Ising models in thermal equilibrium. The Ising model is also used to describe the market game, which closely resembles the battle of the sexes game (Reyes, 2019).

In this paper, we will add to the results obtained in (Hernandez et al., 2013; Hernández et al., 2017; Broere et al., 2017), by deriving analytic results for simple networks using the Ising model. We can compare the results to the results from computational models. The analytically derived results help us to further generalize the conclusions made by these previous studies or help us understand possible deviations from these conclusions. Furthermore, the Ising model allows us to study the effect of correlations introduced by the update rule in the behavior by varying the interaction coefficient $J$.

\subsection{Theory}

The Ising model can be used to calculate analytical expressions for small networks of interacting binary variables. By further generalizing the Ising model, it can be parametrized to represent the asymmetric situation of the battle of the sexes for ring graphs. Before we generalize the Ising model, we will first describe the standard model as illustration of how this model is solved in practice.

\subsubsection{The Ising model and its exact solution in one dimen- sion}

Consider a one-dimensional ring graph $G$. For every node $i$, there is a discrete variable $s_{i}$, such that $s_{i} \in 1,-1$, representing respectively spin up or spin down. So each node is interacting with two neighbours on a line with periodic boundary conditions. Then, the total system's energy is given by

$$
E\left(\left\{s_{i}\right\}\right)=-\sum_{i=1}^{N}\left(J s_{i} s_{i+1}+\frac{B}{2}\left(s_{i}+s_{i+1}\right)\right),
$$

where $\left\{s_{i}\right\}$ denotes the complete set of $s_{i}$ for the number of $N$ (identical) spin particles in an external field $B$, and where $J$ indicates the interaction strength (Ising, 1925; Huang, 2008). Note that on a ring graph we have that $s_{N+1}=s_{1}$. 
The probability that the particle is in the state $s$ is

$$
P\left(\left\{s_{i}\right\}\right)=\frac{e^{-E\left(\left\{s_{i}\right\}\right)}}{Z},
$$

$Z$ being a normalizing constant known as the partition function. In order to get the partition function the sum is made over all possible states of all spins. So the partition function is given by

$$
\begin{aligned}
Z_{N} & =\sum_{s_{1}= \pm 1} \sum_{s_{2}= \pm 1} \ldots \sum_{s_{N}= \pm 1} \exp \left\{\sum_{i=1}^{N}\left[J s_{i} s_{i+1}+\frac{B}{2}\left(s_{i}+s_{i+1}\right)\right]\right\} \\
& =\sum_{s_{1}= \pm 1} \sum_{s_{2}= \pm 1} \ldots \sum_{s_{N}= \pm 1} \prod_{i=1}^{N} \exp \left[J s_{i} s_{i+1}+\frac{B}{2}\left(s_{i}+s_{i+1}\right)\right] \\
& =\sum_{s_{1}= \pm 1} \sum_{s_{2}= \pm 1} \ldots \sum_{s_{N}= \pm 1} \prod_{i=1}^{N} T_{s_{i}, s_{i+1}} .
\end{aligned}
$$

Here we introduced the numbers

$$
T_{s_{i}, s_{i}+1}=\exp \left[J s_{i} s_{i+1}+\frac{B}{2}\left(s_{i}+s_{i+1}\right)\right],
$$

that can be seen as elements of the so called 'transfer matrix' (Kramers and Wannier, 1941a; Kramers and Wannier, 1941b) as

$$
\mathbf{T}=\left(\begin{array}{cc}
T_{1,1} & T_{1,-1} \\
T_{-1,1} & T_{-1,-1}
\end{array}\right)
$$

So the transfer matrix represents the Boltzmann weights of all possible states of $s_{i}$ and $s_{i+1}$.

The partition function is now given by the trace of the $N$-th power of the transfer matrix

$$
Z_{N}=\operatorname{tr}\left[\mathbf{T}^{N}\right]
$$

which can analytically be calculated by diagonalizing the transfer matrix. The total magnetization of the network are given by

$$
M=\frac{\partial}{\partial B} \ln Z_{N}
$$

and the magnetization per spin is thus given by

$$
m=\frac{M}{N} \text {. }
$$




\subsubsection{The generalized Ising model with a node-dependent magnetic field}

The battle of the sexes is an asymmetric game, which means that the same choice combination can have a different payoff for different players. Therefore we need to define attraction (or preference) to accommodate the different preferences of the game. We can do this by allowing the system variables to vary between the particles. The total energy of the system can now be defined as:

$$
E\left(\left\{s_{i}\right\}\right)=-\sum_{i=1}^{N} J_{i+\frac{1}{2}} s_{i} s_{i+1}-\sum_{i=1}^{N} B_{i} s_{i},
$$

where $J_{i+\frac{1}{2}}$ denotes the interaction coefficient between $i$ and $i+1$ and $B_{i}$ can take on different values for different $i$.

The battle of the sexes has two types of players. Looking at Table 4.1a, the row player has a preference for the equilibrium where both players choose $\alpha$ and the column player has a preference for the equilibrium where both players choose $\beta$. When the battle of the sexes is played on a network interaction structure, three types of interactions can occur. A player can interact with a player that has a preference for the opposite equilibrium. This situation is the original battle of the sexes game. However, in a multi-player situation on a network, two players with a preference for $\alpha$ or two players with a preference for $\beta$ can also interact with each other. These situations are symmetric coordination games. Because all three situations need to be accommodated for, we need to define three different transfer matrices. We define the transfer matrix for interactions of opposite preferences

$$
\mathbf{T}=\left(\begin{array}{cc}
T_{1,1} & T_{1,-1} \\
T_{-1,1} & T_{-1,-1}
\end{array}\right)
$$

and for interactions with equal preferences for one type,

$$
\tilde{\mathbf{T}}=\left(\begin{array}{cc}
\tilde{T}_{1,1} & \tilde{T}_{1,-1} \\
\tilde{T}_{-1,1} & \tilde{T}_{-1,-1}
\end{array}\right)
$$

and for the other type,

$$
\hat{\mathbf{T}}=\left(\begin{array}{cc}
\hat{T}_{1,1} & \hat{T}_{1,-1} \\
\hat{T}_{-1,1} & \hat{T}_{-1,-1}
\end{array}\right) .
$$

So the partition function can now be written as product of the trace of the $n$-th power of the different transfer matrices $\mathbf{T}, \tilde{\mathbf{T}}$ and $\hat{\mathbf{T}}$, so schematically,

$$
Z=\operatorname{tr}\left[\mathbf{T}^{n} \tilde{\mathbf{T}}^{\tilde{n}} \hat{\mathbf{T}}^{\hat{n}}\right]
$$


where $n$ refers to number of links with transfer matrix $\mathbf{T}, \tilde{n}$ refers to number of links with transfer matrix $\tilde{\mathbf{T}}, \hat{n}$ refers to number of links with transfer matrix $\hat{\mathbf{T}}$ and the total number of links is $n+\tilde{n}+\hat{n}=N$. In Equation 4.17 we have not explicitly denoted the order of variations of the transfer matrices, but only the total number. However, each transfer matrix represents a link between nodes, so the order of the transfer matrices is dependent on the ordering of the different type of nodes in the network. The ordering is important because the transfer matrices do not commute. In the next section we describe the different orderings of nodes that correspond with different orderings of the transfer matrices. The magnetizations of the network are again given by

$$
M_{i}=\frac{\partial}{\partial B_{i}} \ln Z
$$

whereas the total magnetization per spin is given by

$$
m=\frac{1}{N} \sum_{i} M_{i}
$$

\subsection{Methods}

We compare the results of the Ising model to the results of a previous computational study for four different situations described in Figure 4.1 (Broere et al., 2019). Figure 4.1 illustrates four ring networks with four different distributions of two groups. In the computational set-up the groups are defined as the row and column players in Table 4.1a. Group 1 consists of the row players and is defined by the green nodes in Figure 4.1. Group 1 has a preference for choosing $\alpha$ and the strength of their preference is defined by $S_{1}$. Group 2 consists of the column players and is defined by the red nodes in Figure 4.1. Group 2 has a preference for choosing $\beta$ and the strength of their preference is defined by $S_{2}$. In the Ising model the grouping is the same, only the strength of their preference is dependent on the corresponding value of the magnetic field. The groups can differ on the values of $S$ in the game theory context or $B$ in the Ising context, but the values are the same within the groups. Furthermore, for simplicity we use $J_{i+\frac{1}{2}}=J$ in the Ising model.

In Figure 4.1, network 1 represents a completely integrated network where the nodes only interact with nodes with a different preference. Network 2 represents a completely segregated network that consists of two equal clusters of nodes. Network 3 represents a partly segregated network. Network 4 represents a segregated network with unequally sized groups. 


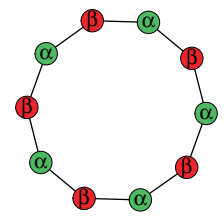

Network 1

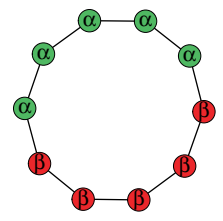

Network 2

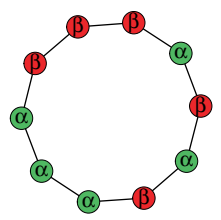

Network 3

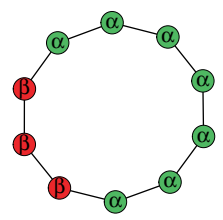

Network 4

Figure 4.1: Networks where the colours indicate the preferences. The green nodes prefer $\alpha$ equilibrium and the red nodes prefer the $\beta$ equilibrium.

We compare the results from the Ising model, with results from computational models. We focus on the computational model described in Broere et al. (2017). In this study nodes play iterated $2 \times 2$ games with their neighbors. Each node gets a preference assigned before the first round of the game ( $\alpha$ or $\beta$ ) that determines whether the node is a row or a column player as shown in Table 4.1a. Every round the nodes choose between $\alpha$ or $\beta$ and the decision of the node is played against all its neighboring nodes. In the first round, the nodes play their preferred behavior with probability one. After every round, the nodes update their probability of playing $\alpha$ or $\beta$ by means of reinforcement learning (Cimini and Sánchez, 2014; Ezaki and Masuda, 2017). The probability of choosing either $\alpha$ or $\beta$ is updated towards what would have been the best choice in the previous round.

\subsection{Results}

In this section we compare the results from the Ising model to the results of the computational model. We will discuss the results of the ring networks with four different distributions of row and column players illustrated in Figure 4.1.

In Figure 4.2, the average magnetization is plotted for the different networks, where the $x$-axis represents the values of $B$ for the red nodes and y-axis the value of $B$ for the green nodes. On the $\mathrm{z}$-axis the average magnetization per spin is plotted represented by the color. Per network, the results are plotted for three values of $J=\{0,0.5,1\}$. These values are chosen because here the most variation occurs.

In the left plots of Figure 4.2, the results are plotted for the networks with $J=0$. Results for $J=0$ are equal to the uncorrelated equilibrium which is the same 

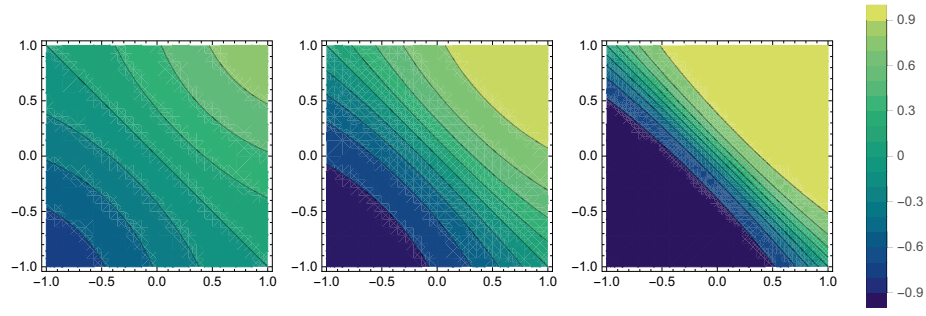

(a) Network 1
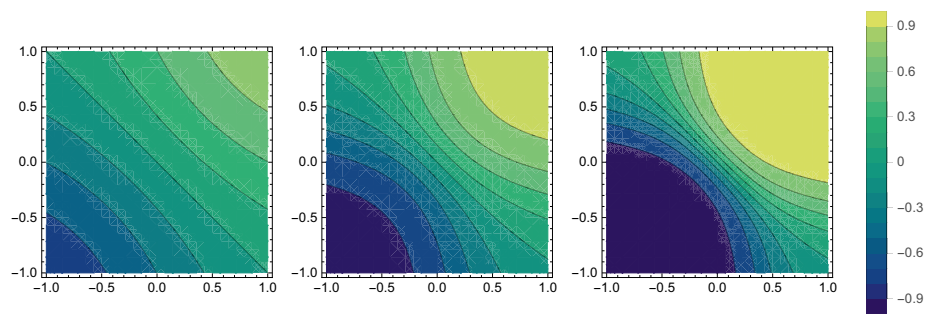

(b) Network 2
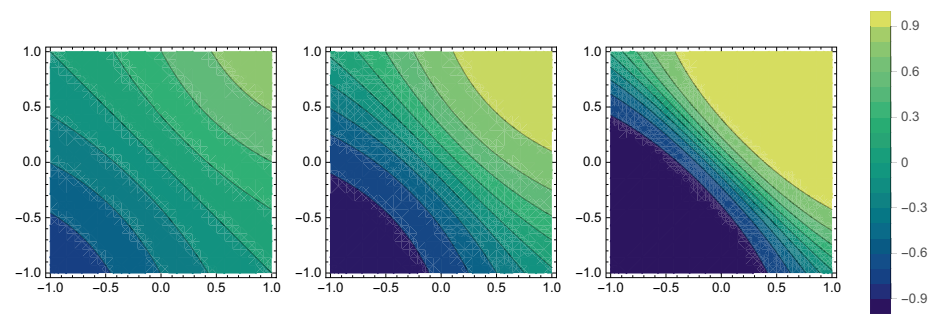

(c) Network 3
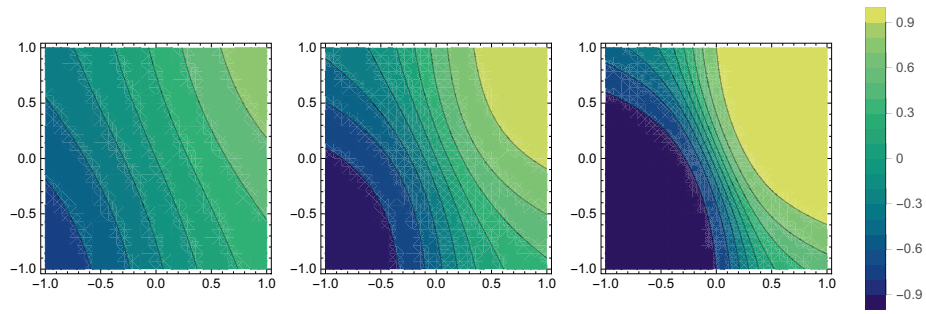

(d) Network 4

Figure 4.2: Average magnetization $m$, where the x-axis represents the $B$ value of red nodes and the y-axis the $B$ value green nodes. On the left the plotted values for $J=0$, in the middle the plotted values for $J=0.5$ and on the right the plotted values for $J=1$. 
for all the networks. On the upper-left to down-right diagonal the $B$-value of the green nodes is exactly opposite to the $B$-value of the red nodes (i.e $B_{\text {green }}=0.5$ and $\left.B_{\text {red }}=-0.5\right)$, the strength of the preferences and the distribution of the preferences in the network are completely in balance for the first three networks. The strength of the preferences cancel each other out, so there is $m=0$ total magnetization. The more $B_{\text {green }}$ and $B_{\text {red }}$ are getting out of balance, the more the magnetization tilts in the direction of the group with a stronger preference. Looking from bottom the left to the top right, this is resulting in a S-shaped distribution. For Network 4 in Figure 4.2 there is a majority preferring $\alpha$. Therefore, the diagonal line is not everywhere equal to $m=0$ magnetization for Network 4 and the magnetization shifts towards the values corresponding with $B_{\text {green }}$.

In the middle plots of Figure 4.2a results are plotted for $J=0.5$ and in the right plots of Figure 4.2 results are plotted for $J=1$. For all networks, the slope of the distribution becomes more steep as $J$ increases. However, the effect of the interaction coefficient is different for the different networks. Comparing the middle Figure 4.2a and the middle Figure 4.2b, the regions are different where the $B$-values have opposite signs. In this region the slope of the distribution is less steep for the middle plot Figure 4.2b compared to the middle plot of Figure 4.2a. The same holds for the right plots of Figure 4.2a and Figure 4.2b. This indicates that it is easier to coordinate on one choice in the integrated network compared to the clustered network. For network 3, the effect of clustering is comparable to the results of network 2. However, the effect of clustering is less prominent, because this network is only partially clustered, as can be seen in Figure 4.2c. For network 4 , the majority group has more influence on the direction of the magnetization than the minority group.

In Figure 4.3, the results of Figure 4.2 are plotted again while the axis are scaled in terms of $S$ according to Equation 4.2 in order to make the plots comparable to the simulation results. As this is now scaled to the computational model, on the zaxis the probability of choosing $\alpha$ is plotted. So a probability of one indicates that all nodes in the network choose $\alpha$, a probability of zero means all nodes choose $\beta$. The first three plots are the results from the Ising model with first results for $J=0$, second $J=0.5$, and third $J=1$ and the most right plot the results of the simulation of the iterated game theory model. Again, results for $J=0$ are equal to the uncorrelated mixed equilibrium solution for the whole network. First thing to notice is that the results are much messier for the simulations, even with higher resolution and more simulations the picture does not change much. 

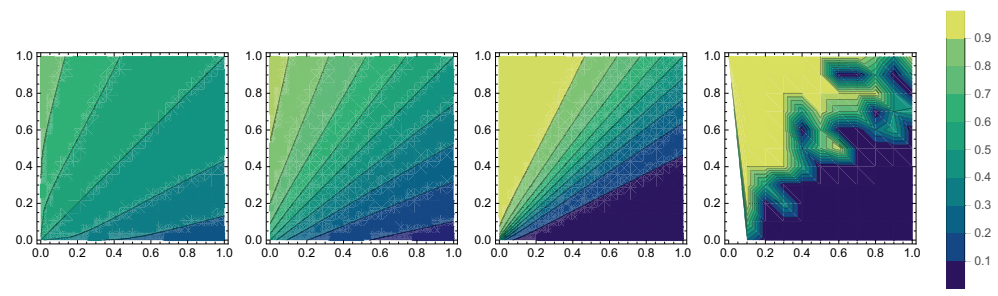

(a) Network 1
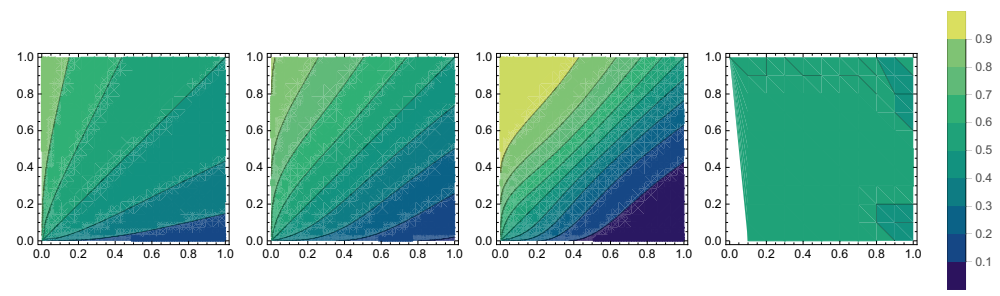

(b) Network 2
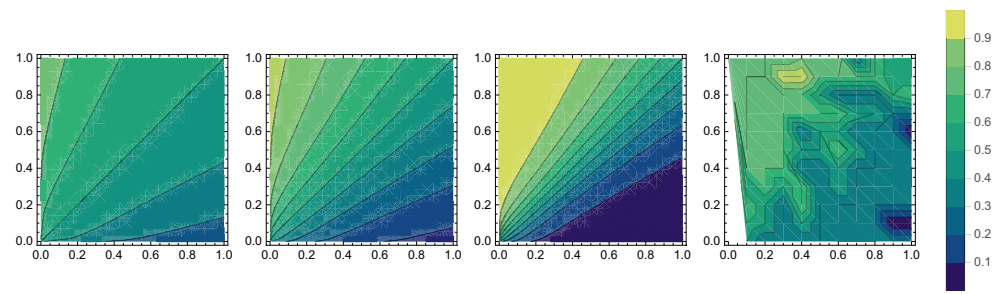

(c) Network 3
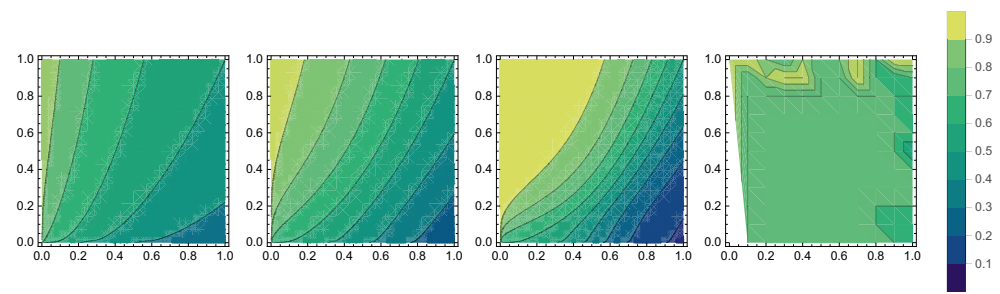

\section{(d) Network 4}

Figure 4.3: Probability of choosing $\alpha$, where the $\mathrm{x}$-axis represents the values of $B_{\text {red }}$ in terms of $S$ and y-axis the value of $B_{\text {green }}$ in terms of $S$. So the x and y axis of Figure 4.2 are converted according to Equation 4.2. On the left the plotted values for $J=0$, in the second lot the values for $J=0.5$, in the third plot the values for $J=1$ and on the right the simulation results 
Although the general pattern seems to be comparable to that of the Ising model, on the diagonal line the pattern looks the same. Eyeballing the results of Network 1 and Network 3, the simulation results seem to be mostly in line with the Ising model results where $J=1$. This seems to indicate that there is a high correlation in behavior in this network. Eyeballing the results of Network 2 and Network 4, the simulation results seem to be most in line with the Ising model results where $J=0$. The results seem to indicate that in more integrated situations like network 1 and network 3, there is more conflict and the correlation can help to overcome the conflicting interests. In the more segregated Network 2 and Network 4 there is less frustration. The segregated groups of nodes can coordinate with each other and therefore the correlation has less of an influence.

\subsection{Conclusions and discussion}

In this paper, we study an asymmetric coordination situation on a network with two methods, an Ising-like model and a computational method. First, we looked at how correlated behavior affected the predictions made by the different models. In the Ising model the correlation in behavior can be modeled by an interaction coefficient. The results are highly dependent on the chosen value of this interaction coefficient. As a general result, the higher the interaction coefficient, the easier the coordination, as reflected in higher magnetization in the Ising model. Comparing the results from the Ising model to the computational method, the effect of the correlation might be larger for situations where there is more frustration. The correlation might helps to facilitate the coordination process in situations where conflict is highest, such as completely integrated networks. In segregated where most nodes only interact with nodes with the same preference, frustration is lowest. When frustration is low the correlation has less influence on the equilibrium because the segregated groups can already coordinate amongst themselves.

At the moment there is no clear version of the Ising model that accurately correspond to the results of the computational model. The ferromagnetic interaction does agree with the high correlation found in the integrated networks. However, the segregated networks appear to correspond better to the results modeled without a ferromagnetic iteration and as a pure strategy (uncorrelated) Nash equlibrium. Intuitively, the problem lies in the fact that the parameters, and particalary the magnetic fields, are geared to represent the battle of the sexes but are clearly suboptimal for the coordination games. Therefore, in order to reproduce the results from the computational method, the most accurate parameters of the current 
formulation of the Ising model are dependent on the network variation. However, in terms of parsimony this dependency is not ideal. In future research it would be interesting to find the microscopic parameters such that the Ising method can mimic the behavior of the computational model independently of the network structure. However it is currently unclear how to parametrize the model such that the same set of parameters yield the same results independent of the network structure. In the current formulation of the Ising model we only looked at dyadic interactions. A possible solution can be to add higher order interactions to the model. However, we leave it to future research to find such a right set of microscopic parameters.

Taking a closer look at the results from the current study, the Ising-like model as a function of all its parameters has a much richer set of equilibria compared to the computational model. One source of this difference is the used update rule in the computational model. The update rule acts as a stepwise optimization algorithm in a multidimensional landscape with (meta)stable and unstable equilibria. Some equilibria obtained by the Ising model are unstable equilibria in the computational method. Because these equilibria are unstable in using the update function, unstable equilibria are not found unless the algorithm by chance starts at this exact spot. The update rule in computational models makes dichotomous decisions at fixed time points. At every time step the probability of choosing one of the two options gets updated towards the best response of the previous round. Because of this dichotomy, the update rule is less sensitive for the size of the payoff difference, as it only evaluates whether or not one decision would yield a better result or not. Even though the update rule adjusts the probability for one decision or the other based on the relative difference in the payoff, many equilibria obtained by the Ising model are less likely found by the update rule. Because, the Ising method yields an analytical expression, the Ising method does not have this problem. This finding corresponds to the study by Hernández, Muñoz-Herrera and Sánchez. As mentioned in the introduction they find a rich set of equilibria where both homogeneous and heterogeneous equilibrium behavior is possible when players have complete information. This set is reduced when players have incomplete information (Hernandez et al., 2013). The update rule used in the computational approach is comparable to the incomplete information case because it only optimizes the payoff by evaluating local information.

Whether the current parametrization of the Ising model for asymmetric preferences or the computational model give better predictions is very much dependent on 
the empirical situation one wants to capture. The computational model predicts situations better where the actors have to make multiple decisions over time and multiple games are played on links of the network. In these situations unstable and metastable equilibria are not very likely and are rightfully excluded from the prediction. In more symmetric situations where the same game is played on every link of the network or when the correlations are externally introduced, the Ising model makes better predictions. In the latter case, i.e., coordination with externally introduced correlations, one may think of a traffic-light coordinating agents in traffic (Correia and Stoof, 2019). It is therefore of utmost importance to be aware of the models' assumptions.

It should be noted that the ring structure we discuss here is an extreme example, because the problems with the iterative method are highest in a ring network. In more structured networks the problem of not ending up in the optimal equilibrium is less of an issue, because most problem occur in completely balanced situations. In less balanced networks, there are less unstable and metastable equilibria and the equilibria are more determined by the structure of the network as earlier research suggest. We can expect to encounter some of these problems in more structured networks as well. However, the results from the current study suggest that the results found in earlier studies would only become stronger if we manage to model them analytically. Also, experimental results corroborate the prediction made by the computational method when human subjects play these games iteratively on structured networks (Broere et al., 2019).

For future work it will be interesting to obtain analytical results for more complex networks by understanding better the correspondence between the various update rules and (possible many-body) interaction parameters of the Ising-like models. Complex networks might reveal a richer set of equilibria in these situations as well. Furthermore, it would be interesting to test the predictions made by these models in empirical situations where we expect different assumptions to be true. This would give an interesting test of the validity of both these models. 


\section{Part II}

\section{Cohesion in dependency and complementary}





\title{
Chapter 5
}

\section{The division of labor and generalized trust: Organic solidarity revisited ${ }^{1}$}

\begin{abstract}
Although Durkheim's theory on organic solidarity is more than 100 years old, the theory has been surprisingly absent in empirical research. In this paper we make a first attempt to study the main mechanisms of the theory empirically. We argue that economic interdependencies and complementarity can serve as a base for generalized trust. We construct variables by means of census data that contains counts of people working in a certain occupation in every metropolitan statistical area in the USA. The constructed variables aim to quantify patterns in the division of labor, which we argue are caused by specialization and the resulting interdependencies between the occupations. Furthermore, we use a novel method from the field of economic complexity that aims to quantify how specialized or 'complex' an occupation is. We test the hypotheses using the General Social Survey data. We show that there are strong relations between both the 'occupational diversity' of the respondents occupation and the 'occupational complexity' of the respondents occupation, on self-reported general trust. The results further strengthen our believe that division of labor is one of the key foundations of human cooperation. We conclude that more empirical research on these mechanisms can improve our understanding of human cooperation.
\end{abstract}

\footnotetext{
${ }^{1}$ This chapter is based on a paper written by Joris Broere, Alje van Dam and Marcus H. Kristiansen. Broere wrote the manuscript, conducted the analyses and contributed substantially to the idea development. Van Dam was consulted on the economic complexity measures, co-wrote the methods section and provided data on the economic complexity measures. Kristiansen contributed substantially to the idea development and contributed substantially to the manuscript. We thank Vincent Buskens and Henk Stoof for their comments on the chapter. This chapter was presented at the Conference on Complex Systems 2018, Thessaloniki, Greece.
} 


\subsection{Introduction}

In recent studies, both Portes and Vickstrom (2011), and Abascal and Baldassarri (2015) call out the need for a better understanding of the mechanisms of organic solidarity. The term 'organic solidarity' was first introduced by Emile Durkheim in his book The division of labor in society (Durkheim, 1893). In this work, Durkheim asks the question: how can a society become more individualistic without losing social order? In the answer to the question, Durkheim distinguishes between two types of solidarity: mechanical solidarity and organic solidarity. Mechanical solidarity is rooted in cohesive and small homogeneous groups or communities whose repeated interactions bring about cooperative norms and values. Organic solidarity on the other hand is founded in interdependencies and complementary between people that emerge from a division of labor. Durkheim claims that the higher the division of labor in a society, the more specialized and individualistic its members. The specialization creates economic dependencies between people that can serve as a basis for trust and solidarity. We argue that both the question and the answer Durkeim poses are still relevant for sociological research today. Although mechanism that form mechanical solidarity are arguably abundantly studied (Evans and Dion, 1991; Axelrod and Hamilton, 1981; Buskens and Raub, 2002), tests of mechanisms that relate to 'organic solidarity' are absent from current empirical literature (Portes and Vickstrom, 2011). In this study we make a first step towards testing aspects of these mechanisms empirically.

The theory of organic solidarity has been absent from the current sociological debate (Portes and Vickstrom, 2011). One explanation could be that organic solidarity is a difficult concept to operationalize, as organic solidarity refers to an evolutionary process, containing many mechanisms at the same time. In this paper we make a first attempt to quantify some of the main mechanisms. We argue that economic interdependencies, caused by specialization, can serve as a base for trust and cooperation. Next, we use census data on the occurrence of 840 categorized occupations in 400 Metropolitan Statistical Areas (MSA) in the US to quantify patterns in the division of labor. We construct two variables that serve as a proxies for economic dependency. First, we construct a variable that measures the average economic diversity of areas in which an occupation occurs. Second, we introduce a novel empirical method from the field of economic complexity that aims to quantify the amount of specialization or 'complexity' of the different occupational categories (Hidalgo and Hausmann, 2009). We show that 'occupational diversity' and 'occupational complexity' emerging from specialization and the division of 
labor are strong predictors of self-reported generalized trust. We conclude that we found evidence in favor of mechanisms of organic solidarity and that these mechanisms deserve to be studied in more detail.

\subsubsection{The division of labor and organic solidarity}

Both Portes and Vickstrom (2011), and Abascal and Baldassarri (2015) argue that Durheims distinction between mechanical and organic solidarity is still relevant. However, according to Portes and Vickstrom (2011) the term 'organic solidarity' has been "largely forgotten in the current debate". Therefore, we start with a small overview of the key concepts as they where originally introduced before turning to its current day relevance.

The division of labor has received attention from several thinkers of which Adam Smith's work is arguably the most well known. Adam Smith concludes in his book The Wealth of the Nations that the division of labor is one of the main drivers of the improvement in productivity of labor (Smith, 1776). The idea behind a division of labor is well established. The production of a product can gain efficiency when the process is separated in different tasks. Workers can specialize in a certain task, making them more competent and efficient in the task at hand, compared to workers who have to divide their time over multiple tasks. The specialization leads to more efficiently produced products, and thus a net higher economic gain. Recent studies focus on mechanisms that could be limiting factors of labor division, such as the cost of managing (or coordinating) specialized workers, principal-agent conflicts, free-riding behavior and communication problems (Becker and Murphy, 1992). However, the general proposition that the returns of time spent on tasks are usually greater to workers who exploit a smaller range of skills is undisputed.

Emile Durkheim later noted that the division of labor was not only instrumental for economic reasons, but also yielded positive effects on what he considered to be 'moral phenomena' (Durkheim, 1893). In Durkheim's treatment on the effects of a division of labor he distinguished between two different forms of solidarity he called mechanical and organic solidarity (Durkheim, 1893). Mechanical solidarity is founded upon homogeneous people whose similarity results in a shared culture and norms that bring about positive outcomes such as trust, cooperation and solidarity. This type of solidarity is mostly present in less developed societies. Its counterpart organic solidarity is rooted in complex interdependencies, complementarity, and individualization that emerge from a division of labor. In societies with a higher division of labor workers have skills that are complementary to each 
other. When jobs become more specialized in subtasks, specialization also creates a higher dependency between people, as there will be a higher need for using each other's service. The interdependency that emerges from a division of labor can function as a basis for social order and solidarity (Durkheim, 1893).

Durkheim has a Darwinian notion on why the development from less developed (homogeneous) societies to more developed (heterogeneous) societies takes place. He argues that specialization and a division of labor is a natural, evolutionary process that happens everywhere in nature and is driven by the struggle to survive. The more organisms look alike, the more they will be each other's rivals. This is because they have the same need and pursue the same goals. However, if organisms differ more from one another, the needs and objectives will also differ, and these will therefore be less of a hindrance to one another. This also applies to forms of society. In primitive forms of society in which people often have the same way of meeting their needs, the common goals can easily come into conflict. If two people have to get their food in the same way, they can regard each other as competition. However, if one of the two decides to collect his food in a different way, there will no longer be any competition (Durkheim, 1893). In more advanced societies with a greater of division of labor, there will be less conflict because different goals are pursued. At the same time, division of labor and specialization make people more dependent on each other, making conflict less rational and requiring cooperation to provide for the full range of necessities of life (Durkheim, 1893). Durkheim compared the functioning of people in a developed society to the functioning of organs in a human body. Every organ in the human body has its own but interdependent task. When one organ stops functioning, the whole body will die. Hence, he called this type of solidarity 'organic solidarity'. (Durkheim, 1893).

Durkheim's work can be placed in a broader literature on social order, starting at Thomas Hobbes's 'state of nature' (Hobbes, 1968). There was, and still is, a popular debate on the question how civil society arose from a state of absolute freedom, and how (forms of) political authority can be legitimate. A popular answer to this question is in the form of the so called social contract theories. Although there are many versions of the social contract theory, they all have one thing in common: people have to give up freedom (or invest in a common good) for the benefit of a commonwealth out of a well-understood self-interest (Christman, 2017). The general argument is to get out of the state of nature and arrive at an orderly state in which life is better for everyone. The agreement among people is 
what is called 'the social contract'. A difficult point within contract theories is the transition from the state of nature to the orderly society. David Hume argues that a social contract has no stable basis without stable social relations (Hume, 1739). Without a basis of trust, a contract offers no guarantee whatsoever. A social contract therefore presupposes a form of order on which to base the contract. In The Division of Labor in Society Durkheim responded to David Hume, claiming that social order can exist without a social contract. Social order can arise from a division of labor. The emergent social order is however instable and needs to be maintained by a social contract.

Although a the term 'social contract' might sound a bit abstract, in practice the word can be replaced by 'institutions', including laws and social rules. A complex division of labor cannot function without well functioning regulating institutions. Historically there is a constant interplay between a changing division of labor and the need for new institutions, and visa versa, how institutions help shape the evolution of a division of labor (North, 1991). Economic growth can for a large part be contributed to the development of institutions that allow an economy to realize gains from specialization and division of labor (North, 1991). The role of institutions in the functioning of societies is of course still very relevant today (Fehr and Gachter, 2000; Kosfeld et al., 2009; Gürerk et al., 2006; IOS, 2019). There is for instance an ongoing debate on how different institutions influence social mechanisms, such as cooperation, trust and solidarity.

Both Portes and Vickstrom (2011), and Abascal and Baldassarri (2015) reintroduce the term 'organic solidarity' to counter what they call Robert Putnam's communitarian conception of social capital. The communitarian conception of social capital often regards social capital to be a property of a group of people, such as a community. The micro mechanisms defining the theories often rely on repeated interactions of cohesive groups that facilitate a shared culture and norms of trust and reciprocity. However, as Putnam concludes himself, communitarian social capital is declining, and modern societal structures are less and less constructed by close cohesive groups (Putnam, 2000). Instead societies are becoming increasingly more complex and groups larger and more abstract. Communitarian explanations can certainly be regarded as one of the mechanisms for the creation of positive collective outcomes such as trust, but probably not the only mechanism. Portes and Vickstrom (2011) argue that given the complexity of modern societies, we should find explanations that go beyond the communitarian conceptualization of social capital that rely on mechanisms rooted in similarity and homogeneity. 
And Abascal and Baldassarri (2015) write: "We should instead set our sights on understanding the building blocks of organic solidarity, the social glue in societies characterized by complex interdependencies, complementarity, and individualization."

Portes and Vickstrom (2011) write that organic solidarity is dependent on three conditions:

1. (Economic) diversity among members of a society.

2. Strong coordinating institutions.

3. A complex division of labor (specialization).

The first two conditions can be regarded are preconditions for the third. A complex division of labor requires a wide set of specialized labor. Strong institutions are needed to regulate a division of labor (North, 1991). There are few examples that influence social interactions of the daily life of people more than these three points. Social interactions are shaped by a division of labor and regulating institutions. However, because these aspects are so intertwined it is difficult to study which of these aspects cause which aspect of social interactions to change. For instance, in testing Durkheims theory, is it the economic dependency emerging from a division of labour, or the associated coordinating institutions that bring about positive effects, such as solidarity cooperation and trust? There are many studies researching the effect of institutions on cooperation and trust (Rothstein and Stolle, 2008; Tan and Tambyah, 2011; Gürerk et al., 2006; Lo Iacono, 2019). In this study we focus on the other two conditions as stated by Portes and Vickstrom (2011). First, we study the effect of 'occupational diversity' on generalized trust. Second, we study the effect of 'occupational complexity' (or specialization) of an occupation on generalized trust.

\subsubsection{Operationalization of organic solidarity}

Mechanisms of organic solidarity are understudied in empirical sociological literature. One explanation could be that organic solidarity is a difficult concept to operationalize. An economic dependency between complementary skilled workers is highly abstract and difficult to measure. This problem is already present in Durkheim's work. Durkheim could not quantify the different forms of social solidarity, therefore he studied solidarity by different types of law that he considered to correspond to the different types of solidarity (Durkheim, 1893). Durkheim 
categorizes criminal law as promoting mechanical solidarity. The corresponding penal law and repressive sanctions are there to maintain a sense of unity and to correct deviations from the communal life and their corresponding values. Civil law characterized by cooperative law and restitutory sanctions is promoting organic solidarity. This type of law is meant to compensate or balance a wrong doing for those who have been harmed. Therefore, restitutory sanctions are there to protect individual rights. According to Durkheim, the balance between restitutory or penal law is an indication of the type of solidarity (Durkheim, 1893). Although the remark has to be made that Durkheim's analysis of these types of law is widely regarded as incorrect by later authors (Baxi, 1974; Merton, 1934; Sheleff, 1975).

In other academic contexts economic dependency is easier quantifiable. There is for instance a wide range of literature on the role of economic interdependencies in international conflict in the field of political science (Gartzke et al., 2001; Wallace, 2013; Copeland, 1996; Krasner, 1976). Trade data between two countries is a perfectly reasonable proxy for economic dependency between countries. There is some well established empirical evidence that countries with a mutual economic dependence participate less often in international conflict with each other, both military and by political means such as sanctioning, compared to countries who don't have a high or only a one directional economic dependence (Gartzke et al., 2001; Wallace, 2013; Copeland, 1996; Krasner, 1976; Walentek et al., 2020; Dekker et al., 2020). These mechanisms are more or less comparable with the mechanism Durkheim describes as organic solidarity. When there is an economic dependency between two actors, the cost of conflict is higher. When the economic dependency goes both ways, this can act as a mutual insurance of the relation.

Although economic interdependency of occupations might be difficult to measure, recently new methods have been developed that aim to quantify patterns of the division of labor between countries economies (Hidalgo and Hausmann, 2009; Tacchella et al., 2012). The method introduced by Hidalgoand Hausmann (2009) tries to measure what is called 'the complexity of a countries economy' and the 'sophistication' or 'complexity' of the products these countries they export. The central idea is that the complexity of an economy can be measured by the capabilities that are required to produce complex products. Some products require more capabilities or sophistication than other products. However, the capabilities of an economy cannot be measured directly, but the products an economy produces can be measured. The method makes use of the idea that some products are related. 
For instance, in order to produce a personal computer, the producer must already have the capability to produce a television. The screen of a personal computer is related to a television screen. Economies that do not possess the capabilities to produce a television are unlikely to produce a personal computer, because a computer is related to a television, but the personal computer is more complex to produce. This reasoning is used to infer the capabilities of economies and complexity of products by analyzing which countries produce which products (Hidalgo and Hausmann, 2009; Tacchella et al., 2012).

In the current study we will apply the same reasoning to determine the complexity of occupations. The assumption made here is that complex occupations need other complex occupations to do their specialized work. So complex occupations can only occur in areas where other complex occupations occur. By means of census data of which occupations occur in which area we infer the complexity of occupations and study its effect on generalized trust. The details of the method will be described in the data and methods section of this study.

\subsection{Theory}

Durkheim's main proposition is that when societies become more specialized, more 'organic' solidarity will emerge because people will become more dependent on each other. So the main independent variable is a division of labor (macro) or individual specialization (micro), while the consequence of a division of labor/specialization is solidarity. There are many different mechanisms one can think of that drive Durkheim's hypothesis and the term 'solidarity' can be interpreted in different ways. We will focus on what we argue is the main mechanism driving this theory: the economic interdependencies that ensure mutual relations between people.

Economic interdependencies ensure the mutual relations between people for the simple reason that defecting is mutually costly (Cournot, 1897). Economic interdependencies can be understood as the behavior needed from multiple individuals in order to achieve a prespecified (economic) objective. The objective cannot be completed as intended if one or more individuals would defect from this behavior. Defection by one or more persons in this process will therefore be costly for everybody involved in the process. The economic costs associated with defecting on a relation someone is economically dependent on will serve as a buffer or threshold for defection. The cost of defection will serve as a basis for mutual trust and cooperative behavior, as the threshold for defection will be higher compared 
to relations without economic dependency. On the individual level one can hypothesize that a mutual economic dependence between two individuals can enforce trust between them. For instance, a wheat farmer is dependent on the baker for turning his product into something valuable and the baker is dependent on the wheat farmer to deliver the wheat to make bread. Defecting or non-cooperative behavior between the wheat farmer and baker can be mutually costly because they are economically dependent on each other. In this two person case, cooperative behavior can still be explained in a relatively easy manner. For instance, cooperation is a Pareto optimal equilibrium in a repeated interactions prisoner's dilemma (Axelrod and Hamilton, 1981). However, this is not limited to a two person case. The more complex a situation becomes, the more interdependencies there are at play (Nowak, 2006; Axelrod and Dion, 1988).

As the division of labor increases, also the number of dependency relations will increase. On a small scale, lets say a community, these relations are still tractable and manageable. However, as group size increases and societies become more complex, the dependency relations become increasingly more abstract and less manageable. A good functioning of oneself becomes dependent on the functioning of your surrounding and visa versa. As the surrounding becomes more complex and abstract, and oneself more specialized, it becomes in one's own self-interest to behave more cooperatively in general.

Another way of thinking about this is from a network perspective. As occupations are usually dependent on (or complementary to) multiple other occupations for their economic performance, and the other occupations are in their turn also dependent on other occupations as well, occupations can be nodes in an dependency/complementarity network. The dependency/complementarity network ensure on a higher level mutual relations and therefore a higher level of general trust. So the following hypothesis can be deduced:

Hypothesis 1: People working in occupations that are more dependent on other occupations for their economic prosperity, will also show higher levels of trust and cooperative behavior.

The problem is however: how to quantify this dependency? Although we cannot measure dependency directly, we can measure several proxies for how specialized and dependent one type of occupation is on others. As stated above, Portes 
and Vickstrom (2011) divide organic solidarity into three mechanisms. In this study we focus on two of the three mechanisms namely, economic diversity and specialization.

If we assume that occupations that are dependent on other occupations for their economic performance also need to be close to each other in physical space (Bettencourt et al., 2014), the follow statement can be deduced: The more dependent an occupation is on other occupations for their economic performance, the higher the diversity (number of different occupations) of the areas this occupation occurs in. In other words, the more occupations are dependent on other occupations, the more they will cluster in heterogeneous areas in physical space. So people with occupations that only occur in highly heterogeneous areas will have higher forms of economic dependency and therefore score higher on general trust. Therefore, we test the following hypothesis:

Hypothesis 1a: People working in occupations that on average occur in areas with more occupational diversity will show higher levels of general trust.

This hypothesis is in line with Durheim's theory that societies that are more heterogeneous should show more organic solidarity.

Furthermore, highly specialized occupations need a diverse set of other occupations for their economic performance, they can only occur in areas with other occupations that are highly specialized themselves. This creates a nested structure where specialized occupations only occur in areas with other occupations. Or as Hildalgo and Hausmann (2009) state it: 'An occupation is as complex as the places it occurs in'. We refer to these types of occupations as being 'economically complex', as they need specialized skills and are dependent on other occupations that require a specialized skill set as well. We therefore formulate the following hypothesis:

Hypothesis 1b: People working in occupations with higher occupational complexity, will show higher levels of general trust.

Note that 'occupational diversity' and 'occupational complexity' are related concepts. In order to be specialized, an occupation needs a diverse set of other oc- 
cupations. However, specialized occupations tend to only occur in diverse areas, where less specialized occupation can occur in diverse areas as well as less diverse areas. So occupations with high diversity occur on average in diverse areas, while occupations with high complexity occur only in diverse areas.

\subsection{Data and methods}

\subsubsection{Data}

We use data that is freely available from the Bureau of Labor Statistics (BLS) in the United States to construct the independent variables to test hypotheses 1a and 1b (BLS, 2019). The data contains counts of people working in a certain occupation as classified by the Standard Occupations Classification (SOC) in every Metropolitan Statistical Area (MSA) in the USA. The data contains 840 occupations on the six digit level and 400 MSA's. Note that the data only takes metropolitan areas into account. MSA's are considered a standard working definition of cities defined by the US Census Bureau, and considered to represent integrated labor markets. The hypothesis will be tested on the General Social Survey (GSS) data. The obtained independent variables from the BLS data are matched to the GSS data by using the occupational codes in both data as an identifier.

\subsubsection{Occupational diversity}

In order to test hypothesis 1a we need to measure the occupational diversity of areas where occupations occur. The diversity can be measured by taking the number of other occupations in an area, where each occupation is weighted relatively to the occurrence in other areas. Next, these occupations are weighted by the relative share of the occupation for which the diversity measure is calculated in that area.

We measure diversity of occupations within a city as the 'effective number of occupations' (Jost, 2006). This measure can be seen as the number of occupations present in a city, corrected for how unequal their distribution is. It is given by the exponential of the Shannon entropy of the distribution of occupations within an MSA. The occupational diversity of a city is thus given by

$$
D(c)=e^{-\sum_{o} p_{o \mid c} \log \left(p_{o \mid c}\right)},
$$


where $p_{o \mid c}=q_{c, o} / \sum_{o} q_{c, o}$ is the share of people with occupation $o$ in city $c$. The average occupational diversity of the cities where a person with occupation $o$ lives is then given by a weighted average

$$
\bar{D}_{o}=\sum_{c} \frac{q_{c, o}}{\sum_{c} q_{c, o}} D(c),
$$

where the diversity of a city $c$ is weighted by the relative share of that city in occupation $o$.

\subsubsection{Occupational complexity}

In order to test hypothesis $1 \mathrm{~b}$ we need to measure the how specialized an occupation is. However, specialization cannot be measured directly. The level of specialization of different occupations will be operationalized using methods from the literature of economic complexity (Hidalgo and Hausmann, 2009). This method assumes that specialized occupations can only occur in areas with other specialized occupations necessary to the occupation in the economic process. For instance, if there a certain amount of lawyers in an area you can expect that there are also judges in that area, the amount of which is relative to the share of lawyers. The other way around, occupations that are less specialized are less bound by their geographical location because they dependent less on other occupations. Therefore, less specialized occupations are more uniformly divided over different areas, while specialized occupations occur in specific places. For instance, a retailer could be considered less specialized, therefore less dependent on other occupations and therefore less bound by geographical location to do business. In what follows we refer to the variable 'occupational complexity' as the empirical operationalization of the level of specialization of an occupation.

The occurrence of an occupation in an area can be represented by means of a bipartite network graph. A bipartite graph contains two types of nodes where where the nodes of one type can only connect to the other type. We construct a bipartite city-occupation network where one type of node represents the occupations and the other type of node represent the MSAs.

The algorithm designed by Hidalgo and Hausmann 2009 aims to summarize the bipartite graph. The algorithm was originally proposed to measure the economic complexity of countries' economies and the level of sophistication for products from world trade data Hidalgo and Hausmann (2009), and later refined by Tacchella et al. (2012). Here we apply the first version to a bipartite city-occupations network 
to extract measures that, to our belief, capture the level of division of labor for cities and occupations. Results for the second version can be found in Table A9 in the appendix of this dissertation.

We define an occurrence of an occupation in a city if the occupation has revealed comparative advantage (RCA) in a certain city. The RCA measures whether a cities' share in a given occupation is higher than the national share of that occupation. Denoting the number of people with occupation $o$ in city $c$ as $q_{c, o}$, we have

$$
R C A_{c, o}=\frac{q_{c, o} / \sum_{o} q_{c, o}}{\sum_{c} q_{c, o} / \sum_{c, o} q_{c, o}} .
$$

A RCA $>1$ tells us that a city specializes in a certain occupation.

Now we can define the city-occupation matrix $M_{c o}$ as

$$
\begin{array}{ll}
M_{c o}=1 & \text { if } R C A_{c, o}>1 \\
M_{c o}=0 & \text { if } R C A_{c, o} \leq 1
\end{array}
$$

From this matrix, shown in Figure A16 of the appendix of this dissertation, we can see an interesting feature: the most diverse cities (i.e. cities that specialize in many occupations) specialize in the occupations that are most rare. In other words, rare occupations only occur in the most diverse cities. On the other hand, cities with low diversity tend to specialize only in occupations that occur in most cities.

In order to construct the variable 'occupational complexity' we describe the bipartite city-occupation network with the iterative algorithm described in Hidalgo and Hausmann 2009. The algorithm is defined in Equations 5.2 and 5.3. We define the 'diversification of a city' $\hat{D}_{c}$ as the sum of all occupations present in that city, weighed by their level of specialization. Hence $\hat{D}_{c}$ is a measure of the 'division of labor' in a city. The level of specialization $S_{o}$ of an occupation in turn is defined in terms of the diversification of the cities it occurs in. Following Bettencourtet al. (2014), we pose that a specialized occupation can only occur in a city with many other occupations, as it can then externalize some of its functions to other occupations while still being able to access these functions. This means that a low diversification of a city is a restriction on the presence of a specialized occupation. 


$$
\begin{aligned}
\hat{D}_{c, i} & =\frac{1}{\hat{D}_{c, 0}} \sum_{o} M_{c o} S_{o, i-1}, \\
S_{o, i} & =\frac{1}{S_{o, 0}} \sum_{c} M_{c o} \hat{D}_{c, i-1} .
\end{aligned}
$$

Due to the nested structure of the city-occupation network, observing an occupation in a city with high diversification (and thus many different occupations) does not give us much information about its level of specialization. On the other hand, observing an occupation occurring in a city with low diversification $\hat{D}_{c}$ means it is unlikely to be highly specialized. Hence the level of specialization $S_{o}$ is given by the reciprocal of the sum of cities it occurs in, weighted by how not specialized these cities are. In other words, the 'generalism' of an occupation increases by its occurrence in cities with low diversification $\hat{D}_{c}$, and the specialization $S_{o}$ is defined as the inverse of this. This results in a heavily discounted level of specialization for occupation that occur in low fitness cities, and a high level of specialization for occupation that only occur in cities with high fitness.

Note that 'diversification of a city' $\hat{D}_{c}$ in Equation 5.2 is almost the same measure as the function $D(c)$ in Equation 5.1. However, Equation 5.2 is a very precise measure of 'occupational diversity' because it does not rely on a binary matrix. Instead, the measure uses the exact counts of occupations in a city. Because both the measures of occupational diversity and occupational complexity rely on the 'diversification of a city', the variables are highly correlated. The Pearson correlation between 'occupational diversity' and 'occupational complexity' is $r=$ 0.64. Because of their conceptual and empirical similarity we will use different regression models in the analyses section of this study. Adding both measures to the same regression model will cause difficult to interpret results.

Summarizing, we define an algorithm that gives a value of division of labor $\hat{D}_{c}$ and specialization $S_{o}$ for every city and occupation respectively, under the assumption that specialization of occupations requires division of labor, and division of labor of a city is measured by the level of specialization of present occupations (Hidalgo and Hausmann, 2009). This circular reasoning is expressed in Equations 5.2 and 5.3. Equations 5.2 and 5.3 are implemented by the EconGeo package in R, which we used for calculation the occupational complexity (Balland, 2017). 


\subsubsection{Analysis strategy}

The hypotheses will be tested on the GSS for the year 2016 (Smith et al., 2018). We use the variable called 'generalized trust' as a dependent variable. The question in the GSS is stated:

- Generally speaking, would you say that most people can be trusted or that you can't be too careful in dealing with people?

The answer categories are:

- Can trust

- Cannot trust

- It depends.

Consistent with previous studies, generalized trust is converted into a binary variable, where the variable is ' 1 ' if the respondent answered "can trust" and ' 0 ' if the respondent answered "cannot trust" or "it depends" (Glaeser et al., 2000). We think this question is well suited to measure trust emerging from 'organic solidarity' mechanisms. Whereas mechanical solidarity should be correlated with in-group trust, while organic solidarity should be correlated with general trust.

We use logistic regression to assess the relation between 'generalized trust' as a dependent variable and 'occupational diversity' and 'occupational complexity' as independent variables. Furthermore, several confounding variables are added to the analyses that we consider to be theoretical confounding variables (Spector and Brannick, 2011; Becker, 2005). All confounding variables are recoded as stated in Glaeser et al. (2000). We add the variables 'education', 'age' and 'sex' as these are confounding variables for job characteristics of the respondent. We add the income of the respondent because the respondent might show higher levels of generalized trust because of a higher economic status. And we add the size of the respondent's residence to the analyses, as city size might be related to generalized trust as well as 'occupational diversity' and 'occupational complexity'. Furthermore, we will use random forest imputation to impute missings on all variables (Stekhoven and Bühlmann, 2011). 
Table 5.1: Logistic regression with dependent variable generalized trust, $\mathrm{N}=2867$.

\begin{tabular}{|c|c|c|c|c|}
\hline & (1) & $(2)$ & $(3)$ & $(4)$ \\
\hline Occupational diversity & $0.054^{* * *}(0.006)$ & & $0.030^{* * *}(0.006)$ & \\
\hline Occupational complexity & & $0.037^{* * *}(0.004)$ & & $0.018^{* * *}(0.004)$ \\
\hline Age & & & $0.033^{* * *}(0.003)$ & $0.035^{* * *}(0.003)$ \\
\hline College educated & & & $1.463^{* * *}(0.100)$ & $1.520^{* * *}(0.101)$ \\
\hline School dropout & & & $-1.349^{* * *}(0.188)$ & $-1.373^{* * *}(0.190)$ \\
\hline Income & & & $0.120^{* * *}(0.026)$ & $0.102^{* * *}(0.025)$ \\
\hline Male & & & $0.545^{* * *}(0.091)$ & $0.604^{* * *}(0.092)$ \\
\hline Town & & & $-0.399(0.216)$ & $-0.247(0.217)$ \\
\hline Small city & & & $-1.069^{* * *}(0.244)$ & $-0.992^{* * *}(0.245)$ \\
\hline Big city & & & $-0.808^{* * *}(0.246)$ & $-0.719^{* *}(0.247)$ \\
\hline Constant & $-11.418^{* * *}(1.139)$ & $-1.631^{* * *}(0.119)$ & $-9.693^{* * *}(1.318)$ & $-4.263^{* * *}(0.410)$ \\
\hline Log Likelihood & $-1,835.838$ & $-1,828.749$ & $-1,503.230$ & $-1,483.348$ \\
\hline Akaike Inf. Crit. & $3,675.676$ & $3,661.498$ & $3,026.460$ & $2,986.696$ \\
\hline
\end{tabular}

\subsection{Results}

In Table 5.1 the logistic regression results are shown with dependent variable self-reported general trust. In the first two models, the bi-variate relations between occupational diversity and occupational complexity are shown. In models 3 and 4 confounding variables are added to the models. In the first model there is a statistical significant effect of occupational diversity on general trust, $b=0.054, z=9.569, p<0.001$. This indicates that respondents who have an occupation that on average occurs in more diverse places are more likely to answer the trust question positive. The odds for answering the trust question positive is 43 times higher for the person with the highest score on occupational diversity compared to the person with the lowest score in the data. In model 3, several confounding variables are added. Also here there is a statistical significant effect of occupational diversity on trust, $b=0.030, z=4.786, p<0.001$. After adding confounding variables the odds for answering the trust question positive is 8 times higher for the person with the highest score on occupational diversity compared to the person with the lowest score in the data. We therefore have evidence in favor of hypothesis $1 \mathrm{a}$.

In model 2 , there is a statistically significant effect of occupational complexity on general trust, $b=0.037, z=9.519, p<0.001$. This indicates that respondents that have an occupation that scores higher on occupational complexity are more likely to answer the trust question positive. The odds for answering the trust 
question positive is 37 times higher for the person with the highest possible score on occupational complexity compared to the person with the lowest possible score. In model 4 several confounding variables are added. Also here there is a statistical significant effect of occupational complexity on trust, $b=0.019, z=4.365, p<$ 0.001. After adding the confounding variables the odds for answering the trust question positive is 6 times higher for the person with the highest possible score on occupational complexity compared to the person with the lowest possible score. We therefore have evidence in favor of hypothesis $1 \mathrm{~b}$.

Furthermore we find positive effects of age, education, income, and being male. All these effects are in line with previous literature.

The analyses are robust for the alternative formulation of the occupational complexity measure by Tacchella et al., (2012), and other choices for the RCA value. These results can be found in Table A9 and Table A10 of the appendix.

\subsection{Conclusion and discussion}

Portes and Vickstrom (2011) put forward three conditions that together form organic solidarity. In this study we presented a first empirical test of two of those conditions. The effect of institutions on different operationalizations of social order, such as generalized trust and cooperation, have been extensively studied. We studied the effect of two other aspects of organic solidarity on generalized trust. We found a large effect of the occupational diversity of a respondent on selfreported generalized trust. This indicates that respondents who have occupations that occur in areas where there are on average many other occupations as well, the respondent is more likely to trust. Second, we found a large effect of the occupational complexity of an occupation on self-reported generalized trust. These findings corroborate the hypotheses put forward by Portes and Vickstrom (2011).

We believe that a division of labor can serve as a key foundation of human cooperation. Everyday life exhibits a wide spread of trust and cooperation that exceeds explanations of homogeneity, repeated interactions and community groups. Economic differentiation and organic integration coordinates daily life, and is controlled by an institutional context. They can provide explanations of trust and cooperation in modern societies in which many interactions are incidental and not with recurring partners.

The chosen operationalizations of diversity, complexity, and trust are still very 
abstract. Both dependent and independent variables lack empirical clarity and theoretical rigor. The dependent variable 'generalized trust' cannot be explained with 'communitarian' theories. Communitarian theories are inherently about repeated interactions and mechanisms within the community, while general trust refers to interactions with people you do not know. So, generalized trust inherently exceeds communitarian theories. However, if we choose to take the theory of organic solidarity seriously, we need new micro models that explain how economic embeddedness leads to generalized trust. An important part is to rethink how generalized trust is different from other types of trust that are related to communitarian or in-group trust mechanisms. The operationalization of generalized trust in the general social survey is a one-dimensional, superficial representation of trusting behavior in every moment in the daily life of people. As Portes and Vickstrom (2011) already point out, when an individual in any modern metropolis gets into a crowded metro, that individual is exercising an tremendous amount of trusting behavior without any previous interaction or communication. Yet, all these types of interactions do not lead to problems in the vast majority of times. In order to explain this, we need a multidimensional scale of generalized trust, or organic trust. We need to theorize about how this type of trust differs from communitarian or in-group based types of trust. Next, we need to formulate hypotheses on when to expect communitarian trust mechanisms to happen and when to expect organic trust mechanism to happen and test these hypotheses empirically.

Furthermore, on the side of the independent variables, this study is by no means a causal test of the theory of organic solidarity. The chosen operationalizations are operationalizations on the occupational or meso level. How exactly specialization of individuals can lead to higher general trust is unclear. It is difficult to entangle what causes which effect, as institutions cause social relations to change, but changing social relations also cause institutions to change. Furthermore, the 'occupational complexity' measure is far from a perfect proxy for specialization or economic interdependence. It is hard to pinpoint what it exactly measures, although there is some concept validity because it correlates highly with education, income and the dependent variable. However, the measure 'occupational diversity' makes use of population counts from census data. Occupational diversity is therefore a near perfect measure of what we want it to measure. However, it is unclear how 'occupational diversity' can cause more general trust directly. Therefore, organic solidarity still is subject to underdetermination, both empirically and theoretically. 
Future research needs to focus on getting better measures and theoretical clarity of both dependent and independent variables. However, we do believe that the current results indicate that organic solidarity is worth the reconsideration and should be studied in more detail empirically. 

Appendices 



\section{Appendices Chapter 2}

Table A1: Regression results, standardized, dependent variable Power, with deterministic myopic best response

\begin{tabular}{lccccccc}
\hline \hline & ER & SW $p=0.25$ & SW $p=0.20$ & SW $p=0.15$ & SW $p=0.1$ & SW $p=0.05$ & PA \\
\hline Even & 0.139 & 0.138 & 0.140 & 0.143 & 0.137 & 0.141 & 0.138 \\
DegC & & 0.268 & 0.251 & 0.187 & 0.160 & 0.162 & 0.329 \\
EVC & 0.114 & & & & & & \\
BetC & 0.428 & & & & & & \\
ClosC & -10.997 & & & & & & \\
Constant & 0.829 & 0.605 & 0.611 & 0.612 & 0.609 & 0.592 & 0.586 \\
\hline $\mathrm{N}$ & 20,000 & 4,000 & 4,000 & 4,000 & 4,000 & 4,000 & 20,000 \\
$\mathrm{R}^{2}$ & 0.608 & 0.666 & 0.658 & 0.668 & 0.638 & 0.620 & 0.642 \\
\hline \hline
\end{tabular}

${ }^{*}$ Even $=$ variable indicating an even degree, $E V C=$ Eigenvector centrality, BetC=Betweenness centrality,

DegC $=$ Degree centrality, Clos $C=$ Closeness centrality. ${ }^{*}$ Interaction in uncentered variables. 


\section{Supplementary methods, Centrality measures}

Degree centrality is the number of edges a node has. For a given graph $G:=(V, E)$ comprising a set $V$ of nodes together with a set $E$ of edges, let $\boldsymbol{A}$ be the adjacency matrix, i.e. $\left(A_{i j}\right)=1$ if node $i$ is linked to node $j$, and $\left(A_{i j}\right)=0$ otherwise. Then the degree centrality is defined as:

$$
k_{i}=\sum_{j} A_{i j}
$$

Eigenvector centrality is the centrality score of a node weighted for the centrality scores of the neighboring nodes. The relative centrality score of a node can be defined as:

$$
x_{i}=\frac{1}{\lambda} \sum_{j \in G} A_{i j} x_{j},
$$

where $\lambda$ is a constant. With a rearrangement this can be written in vector notation as the eigenvector equation:

$$
\boldsymbol{A x}=\lambda \boldsymbol{x}
$$

where the eigenvector centrality scores correspond to the largest eigenvector of the graph adjacency matrix.

Betweenness centrality of node $i$ measures the fraction of shortest paths that pass through a node in the network defined as:

$$
C_{B}(i)=\frac{1}{(N-1)(N-2)} \sum_{s \neq i \neq r} \frac{\sigma_{s, r(i)}}{\sigma_{s, r}},
$$

where $\sigma_{s, r}$ is the shortest paths between source node $s$ and target node $r, \sigma_{s, r(i)}$ is the number of shortest paths between source node $s$ and target node $r$ that pass through $i$, and $N$ is the number of nodes in the network.

Closeness centrality measures the average shortest distance from one node to another in a network, defined as:

$$
C_{C}(i)=\frac{1}{(N-1)} \frac{1}{\sum_{r} d(r, i)},
$$

where $d(r, i)$ is the distance between nodes $i$ and $r$. 
Both degree centrality and betweenness centrality will be normalized over the different networks to make between-network comparison possible. Normalization is defined by:

$$
\operatorname{normal}(C(i))=\frac{C(i)-\min (C)}{\max (C)-\min (C)},
$$

where $\max (C)$ is the maximum centrality value over all nodes in all networks in the simulation and $\min (C)$ is the minimum centrality value over all nodes in all networks in the simulation. 
Figure A1: Variance of the proportion of times a node converges to the preferred equilibrium state, played on a network when the same initial distribution is played 100 times on the same network. A proportion of 1 indicates the node always converges to the preferred equilibrium state, 0 indicates the node never converges to the preferred equilibrium state.

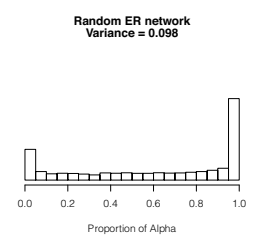

(a)

Small world, $p=0.2$
Variance $=0.095$

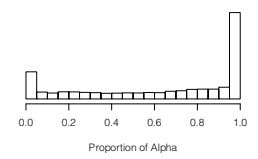

(c)

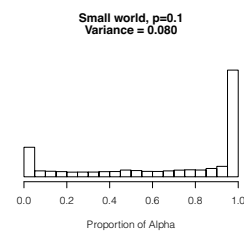

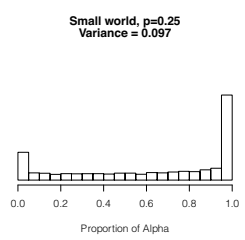

(b)

Small world, $p=0.15$
Variance $=0.088$

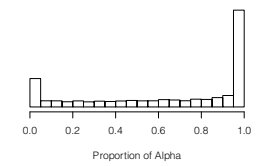

(d)

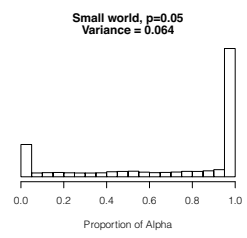

(e)

(f)

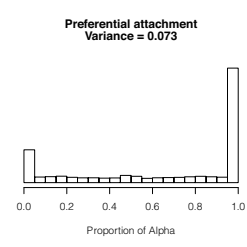

(g) 
Figure A2: Left the network and right the estimate of node Power after playing different starting conditions on the same network. The lines indicate the proportion of times that the node ends in the preferred equilibrium.
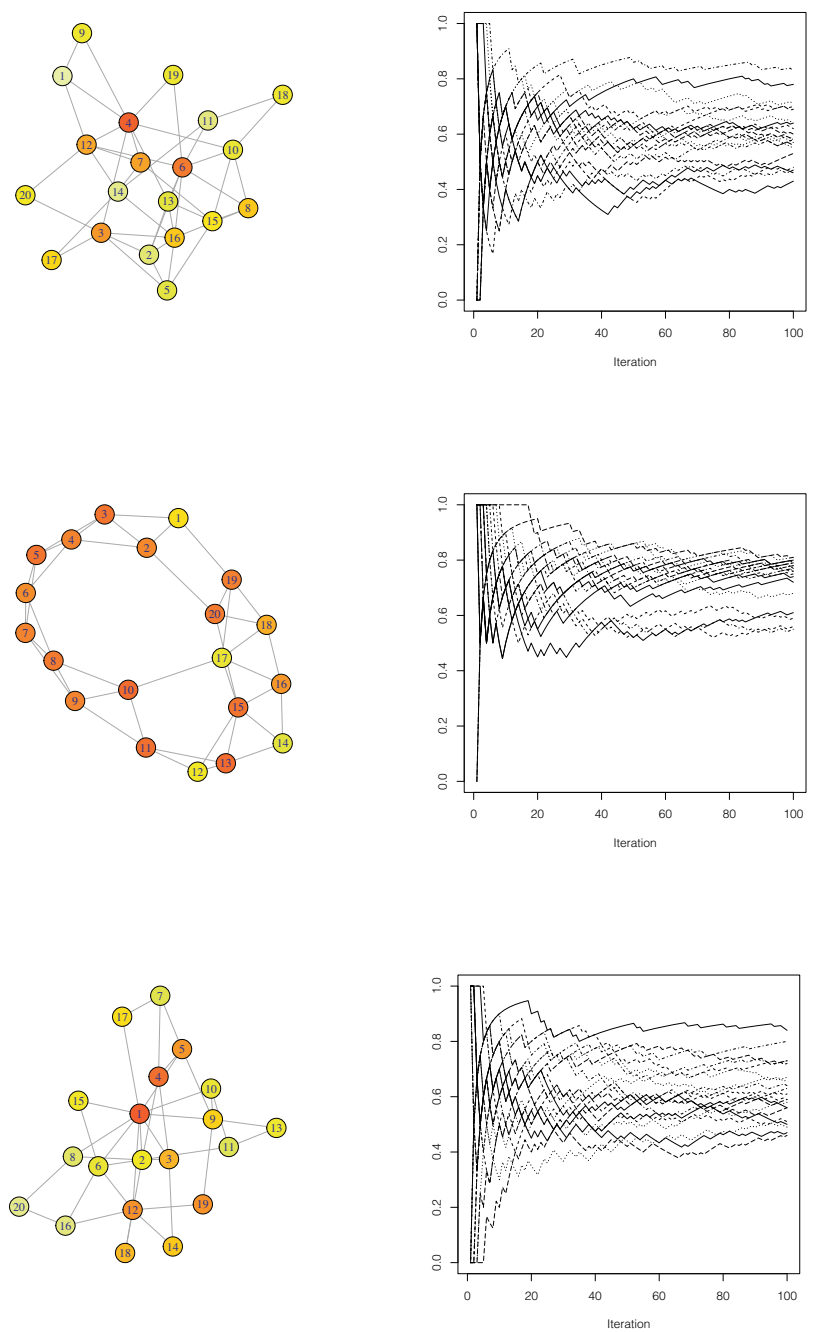
Figure A3: Average heterogeneity over 200 replications (except for $\mathrm{N}=640$ : 50 replications; $\mathrm{N}=1280$ : 10 replications $*$, network density $=0.1$ The larger networks are computationally very intensive, therefore we used less replications for the network sizes $\mathrm{N}=640, \mathrm{~N}=1280$. In addition, less replications are necessary because there is less variation between networks if network size is larger.

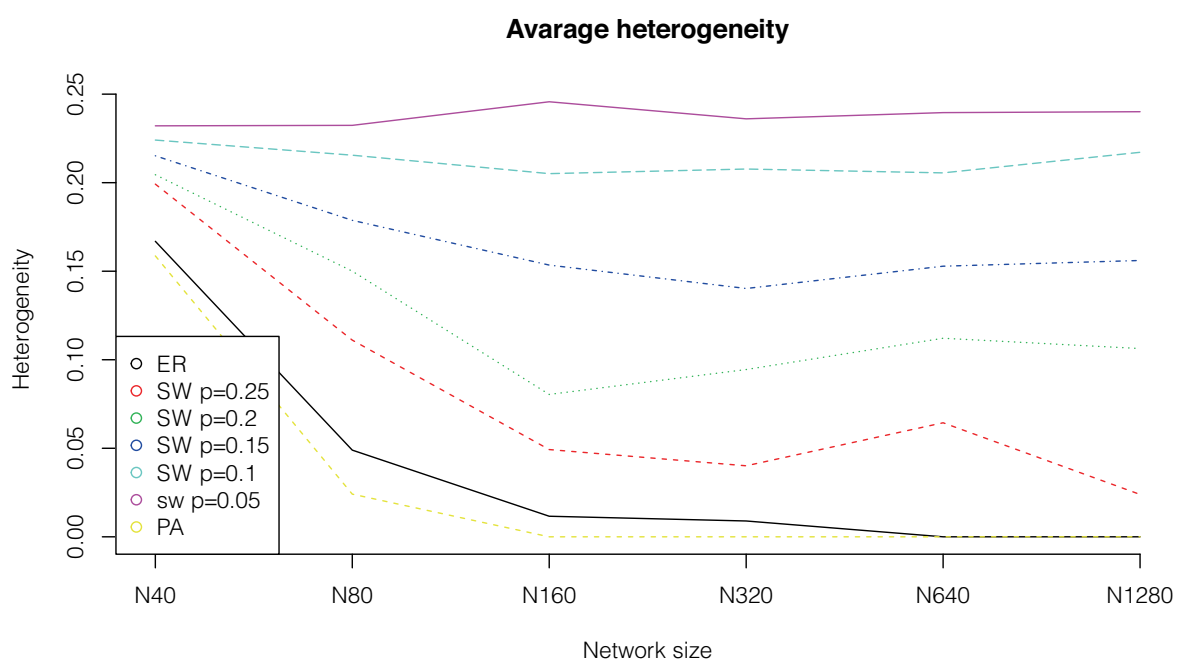


Figure A4: Average heterogeneity over 200 replications (except for $\mathrm{N}=640$ : 50 replications; $\mathrm{N}=1280$ : 10 replications $*$, network density $=0.2$. The larger networks are computationally very intensive, therefore we used less replications for the network sizes $\mathrm{N}=640, \mathrm{~N}=1280$. In addition, less replications are necessary because there is less variation between networks if network size is larger.

\section{Avarage heterogeneity}

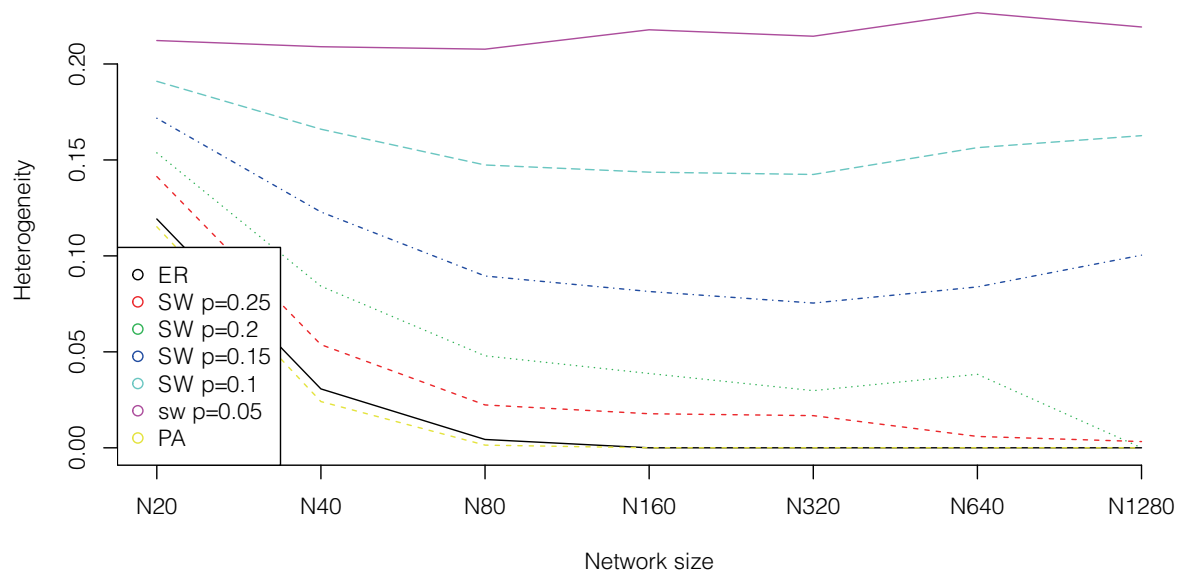


Figure A5: Average heterogeneity over 200 replications (except for $\mathrm{N}=640$ : 50 replications; $\mathrm{N}=1280$ : 10 replications $*$, network density $=0.3$. The larger networks are computationally very intensive, therefore we used less replications for the network sizes $\mathrm{N}=640, \mathrm{~N}=1280$. In addition, less replications are necessary because there is less variation between networks if network size is larger.

\section{Avarage heterogeneity}

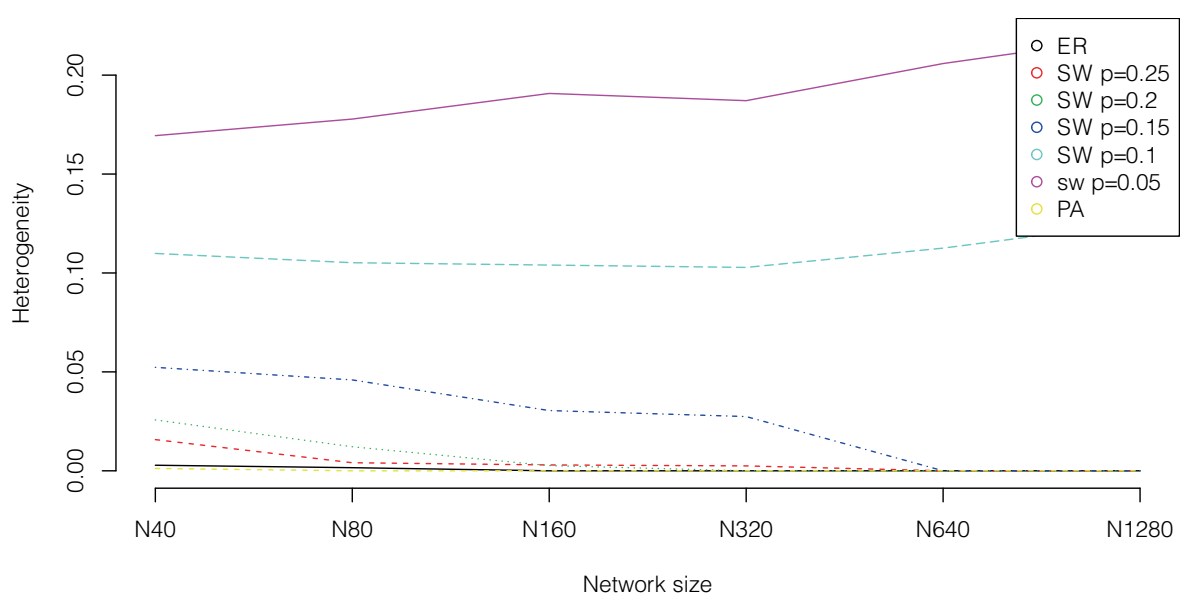


Table A2: Regression results, standardized, dependent variable Power, $\mathrm{N}=40$.

\begin{tabular}{|c|c|c|c|c|c|c|c|}
\hline & $\mathrm{ER}$ & SW $p=0.25$ & SW $p=0.20$ & SW $p=0.15$ & SW $p=0.1$ & $\mathrm{SW} p=0.05$ & $\mathrm{PA}$ \\
\hline Even & & 0.053 & 0.061 & 0.071 & 0.078 & 0.091 & 0.023 \\
\hline DegC & -0.006 & 0.210 & 0.215 & 0.190 & 0.116 & 0.065 & 0.316 \\
\hline EVC & 0.153 & & & & & & \\
\hline BetC & 0.117 & & & & & & \\
\hline Constant & 0.424 & 0.409 & 0.421 & 0.466 & 0.517 & 0.554 & 0.452 \\
\hline $\mathrm{N}$ & 20,000 & 4,000 & 4,000 & 4,000 & 4,000 & 4,000 & 20,000 \\
\hline $\mathrm{R}^{2}$ & 0.361 & 0.391 & 0.370 & 0.354 & 0.367 & 0.417 & 0.532 \\
\hline
\end{tabular}

Table A3: Regression results, standardized, dependent variable Power, $\mathrm{N}=80$.

\begin{tabular}{|c|c|c|c|c|c|c|c|}
\hline & ER & SW $p=0.25$ & $\mathrm{SW} p=0.20$ & SW $p=0.15$ & $\mathrm{SW} p=0.1$ & SW $p=0.05$ & $\mathrm{PA}$ \\
\hline Even & & 0.053 & 0.010 & 0.014 & 0.053 & 0.091 & \\
\hline $\operatorname{DegC}$ & 0.070 & 0.225 & 0.161 & 0.156 & 0.106 & 0.060 & 0.205 \\
\hline EVC & 0.042 & & & & & & \\
\hline BetC & 0.064 & & & & & & \\
\hline BetC:EVC & 0.033 & & & & & & \\
\hline Constant & 0.420 & 0.423 & 0.444 & 0.463 & 0.507 & 0.548 & 0.465 \\
\hline $\mathrm{N}$ & 20,000 & 4,000 & 4,000 & 4,000 & 4,000 & 4,000 & 20,000 \\
\hline $\mathrm{R}^{2}$ & 0.272 & 0.211 & 0.182 & 0.161 & 0.158 & 0.152 & 0.401 \\
\hline
\end{tabular}

${ }^{*}$ Even = variable indicating an even degree, EVC= Eigenvector centrality, BetC= Betweenness centrality, DegC = Degree centrality, Clos $C=$ Closeness centrality. ${ }^{*}$ Interaction in uncentered variables. 
Figure A6: Proportion of $\alpha$ played in a network after convergence for $\mathrm{S}=0.7$. (a) for random ER-Networks, (b) for small-world Networks with rewiring probability 0.25 , (c) for small-world Networks with rewiring probability 0.2 , (d) for smallworld Networks with rewiring probability 0.15, (e) for small-world Networks with rewiring probability 0.1 , (f) for small-world Networks with rewiring probability 0.05, (g) for PA-networks, (h) within clusters of all types of networks.

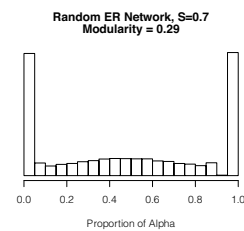

(a)

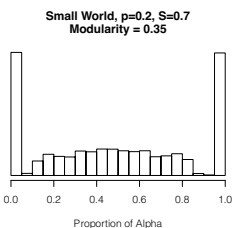

(c)

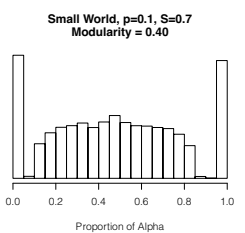

(e)

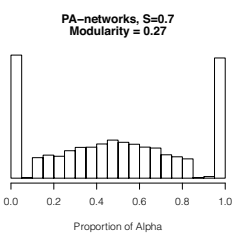

(g)

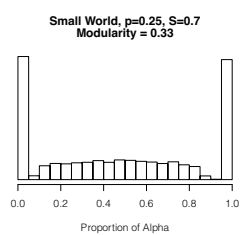

(b)

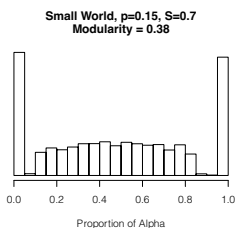

(d)

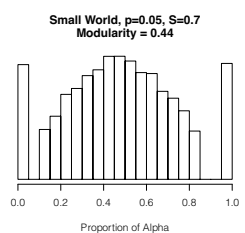

(f)

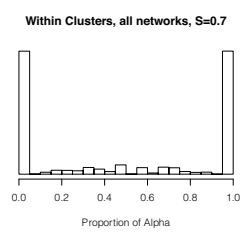

(h) 
Figure A7: Proportion of $\alpha$ played in a network after convergence for $\mathrm{S}=0.5$. (a) for random ER-Networks, (b) for small-world Networks with rewiring probability 0.25 , (c) for small-world Networks with rewiring probability 0.2 , (d) for smallworld Networks with rewiring probability 0.15, (e) for small-world Networks with rewiring probability 0.1 , (f) for small-world Networks with rewiring probability 0.05, (g) for PA-networks, (h) within clusters of all types of networks.

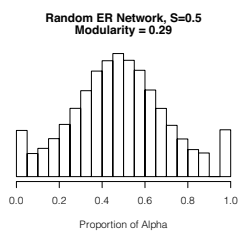

(a)

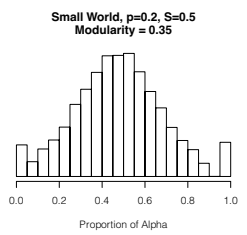

(c)

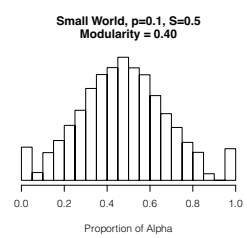

(e)

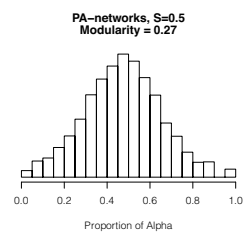

(g)

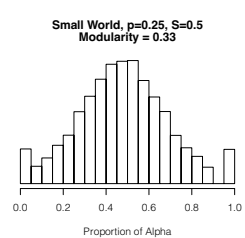

(b)

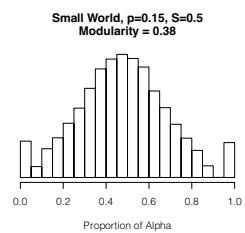

(d)

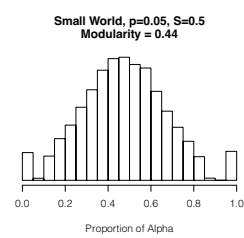

(f)

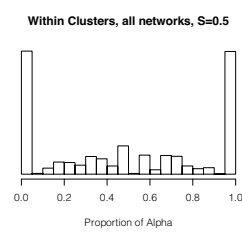

(h) 
Table A4: Regression results, standardized, dependent variable Power, with $\mathrm{S}=0.5$

\begin{tabular}{lccccccc}
\hline \hline & ER & SW $p=0.25$ & SW $p=0.20$ & SW $p=0.15$ & SW $p=0.1$ & SW $p=0.05$ & PA \\
\hline Even & -0.041 & -0.087 & -0.096 & -0.102 & -0.106 & -0.102 & -0.082 \\
DegC & 1.179 & 0.225 & & -0.012 & 0.020 & 0.105 & 0.240 \\
ClosC & 0.324 & & & & & \\
BetC & 0.211 & 0.246 & 0.270 & 0.202 & 0.141 & & \\
EVC & -0.132 & & & & & & \\
DegC:ClosC & -1.401 & & & & & & \\
DegC:EVC & 0.034 & & & & & & \\
Constant & 0.603 & 0.810 & 0.816 & 0.820 & 0.813 & 0.789 & 20,000 \\
\hline $\mathrm{N}$ & 20,000 & 4,000 & 4,000 & 4,000 & 4,000 & 4,000 & 0.535 \\
$\mathrm{R}^{2}$ & 0.407 & 0.371 & 0.412 & 0.475 & 0.483 & 0.428 & 0.535 \\
\hline \hline
\end{tabular}

${ }^{*} E V C=$ Eigenvector centrality, BetC=Betweenness centrality, DegC $=$ Degree centrality, Clos $C=$ Closeness centrality *Interaction in uncentered variables

Table A5: Regression results, standardized, dependent variable Power, with $\mathrm{S}=0.7$

\begin{tabular}{lccccccc}
\hline \hline & ER & SW $p=0.25$ & SW $p=0.20$ & SW $p=0.15$ & SW $p=0.1$ & SW $p=0.05$ & PA \\
\hline Even & 0.137 & 0.053 & 0.153 & 0.156 & 0.166 & 0.171 & 0.135 \\
DegC & -0.172 & 0.272 & 0.265 & 0.203 & 0.152 & 0.113 & 0.489 \\
EVC & -0.099 & & & & & \\
BetC & 0.358 & & & & & & \\
EVC:DegC & 0.426 & & & & & & \\
Constant & 0.543 & 0.460 & 0.469 & 0.487 & 0.513 & 0.526 & 0.516 \\
\hline $\mathrm{N}$ & 20,000 & 4,000 & 4,000 & 4,000 & 4,000 & 4,000 & 20,000 \\
$\mathrm{R}^{2}$ & 0.546 & 0.614 & 0.632 & 0.627 & 0.652 & 0.650 & 0.522 \\
\hline \hline
\end{tabular}

${ }^{*} E V C=$ Eigenvector centrality, BetC=Betweenness centrality, DegC $=$ Degree centrality, Clos $C=$ Closeness centrality *Interaction in uncentered variables 


\section{Appendices Chapter 3}

\section{Specification computational model}

The $2 \times 2$ game can be mapped on a network by pairwise interactions between nodes who share an edge. In each round the nodes update their belief on what strategy yields the highest payoff, $\alpha$ or $\beta$, by means probabilistic dynamic in which the behavioral propensity changes towards the best response. Let $i=1 \ldots N$ be the nodes in the population. Let $s \alpha, \beta\}$ be the strategy of node $i, \pi_{i}$ the payoff of node $i$ and $\tilde{\pi}_{i}$ the payoff when the alternative strategy would have been played. Then, the probability $p_{s, i}^{t}$ that a strategy $s$ is played in round $t$, given the probability $p_{s, i}^{t-1}$ that a strategy $s$ is played in round $t-1$ equals;

$$
p_{s, i}^{t}= \begin{cases}p_{s, i}^{t-1}+0.5 & \text { for } \pi_{i}^{t-1} \geq \tilde{\pi}_{i}^{, t-1} \\ p_{s, i}^{t-1}-0.5 & \text { for } \pi_{i}^{t-1}<\tilde{\pi}_{i}^{, t-1}\end{cases}
$$

where $p_{s, i}^{t}$ is the probability that strategy $s$ is played at some time $t$ by node $i$ (Buskens and Snijders, 2015). So, at every time $t$ each node updates the probability to play $\alpha$ or $\beta$ towards a myopic best response reply strategy. If the best reply at time $t-1$ would have been $\alpha$, the probability of playing $\alpha$ at time $t$ increases compared to time $t-1$. If the best reply at time $t-1$ would have been $\beta$, the probability of playing $\alpha$ at time $t$ decreases at time $t$ (simultaneously the probability to play $\beta$ increases). The probabilities are naturally bounded by the values 0 and 1. Note: We used a step of 0.5 instead of 0.1 , because we think this is more realistic for human subjects 
Table A6: Adjacency matrix of the random network

$\begin{array}{llllllllllllllllllll}0 & 1 & 0 & 0 & 0 & 0 & 0 & 0 & 1 & 0 & 0 & 0 & 0 & 0 & 0 & 0 & 0 & 0 & 0 & 0 \\ 1 & 0 & 0 & 0 & 1 & 0 & 1 & 1 & 0 & 0 & 0 & 0 & 0 & 0 & 0 & 0 & 1 & 0 & 0 & 0 \\ 0 & 0 & 0 & 0 & 0 & 0 & 0 & 0 & 0 & 0 & 1 & 0 & 0 & 0 & 1 & 0 & 1 & 0 & 0 & 0 \\ 0 & 0 & 0 & 0 & 0 & 0 & 0 & 1 & 1 & 0 & 0 & 0 & 1 & 0 & 0 & 0 & 0 & 0 & 0 & 0 \\ 0 & 1 & 0 & 0 & 0 & 0 & 0 & 0 & 0 & 0 & 0 & 0 & 0 & 1 & 0 & 0 & 1 & 0 & 1 & 0 \\ 0 & 0 & 0 & 0 & 0 & 0 & 0 & 0 & 0 & 0 & 0 & 1 & 0 & 0 & 0 & 0 & 0 & 0 & 0 & 1 \\ 0 & 1 & 0 & 0 & 0 & 0 & 0 & 0 & 0 & 1 & 0 & 0 & 0 & 0 & 1 & 0 & 0 & 0 & 1 & 0 \\ 0 & 1 & 0 & 1 & 0 & 0 & 0 & 0 & 0 & 0 & 0 & 1 & 0 & 0 & 0 & 1 & 0 & 0 & 0 & 0 \\ 1 & 0 & 0 & 1 & 0 & 0 & 0 & 0 & 0 & 1 & 0 & 0 & 1 & 0 & 0 & 1 & 0 & 0 & 0 & 1 \\ 0 & 0 & 0 & 0 & 0 & 0 & 1 & 0 & 1 & 0 & 1 & 0 & 1 & 0 & 0 & 1 & 1 & 0 & 0 & 0 \\ 0 & 0 & 1 & 0 & 0 & 0 & 0 & 0 & 0 & 1 & 0 & 0 & 1 & 1 & 0 & 0 & 0 & 0 & 0 & 0 \\ 0 & 0 & 0 & 0 & 0 & 1 & 0 & 1 & 0 & 0 & 0 & 0 & 1 & 0 & 1 & 0 & 0 & 0 & 0 & 0 \\ 0 & 0 & 0 & 1 & 0 & 0 & 0 & 0 & 1 & 1 & 1 & 1 & 0 & 0 & 0 & 0 & 1 & 0 & 0 & 0 \\ 0 & 0 & 0 & 0 & 1 & 0 & 0 & 0 & 0 & 0 & 1 & 0 & 0 & 0 & 0 & 0 & 0 & 0 & 0 & 0 \\ 0 & 0 & 1 & 0 & 0 & 0 & 1 & 0 & 0 & 0 & 0 & 1 & 0 & 0 & 0 & 0 & 0 & 1 & 0 & 0 \\ 0 & 0 & 0 & 0 & 0 & 0 & 0 & 1 & 1 & 1 & 0 & 0 & 0 & 0 & 0 & 0 & 1 & 0 & 1 & 0 \\ 0 & 1 & 1 & 0 & 1 & 0 & 0 & 0 & 0 & 1 & 0 & 0 & 1 & 0 & 0 & 1 & 0 & 1 & 0 & 1 \\ 0 & 0 & 0 & 0 & 0 & 0 & 0 & 0 & 0 & 0 & 0 & 0 & 0 & 0 & 1 & 0 & 1 & 0 & 0 & 0 \\ 0 & 0 & 0 & 0 & 1 & 0 & 1 & 0 & 0 & 0 & 0 & 0 & 0 & 0 & 0 & 1 & 0 & 0 & 0 & 0 \\ 0 & 0 & 0 & 0 & 0 & 1 & 0 & 0 & 1 & 0 & 0 & 0 & 0 & 0 & 0 & 0 & 1 & 0 & 0 & 0\end{array}$


Table A7: Adjacency matrix of the clustered network

$\begin{array}{llllllllllllllllllll}0 & 1 & 1 & 0 & 0 & 0 & 0 & 0 & 0 & 0 & 0 & 0 & 0 & 0 & 0 & 0 & 0 & 0 & 1 & 1 \\ 1 & 0 & 1 & 1 & 0 & 0 & 0 & 0 & 0 & 0 & 0 & 0 & 0 & 0 & 0 & 0 & 0 & 0 & 0 & 1 \\ 1 & 1 & 0 & 1 & 1 & 0 & 0 & 0 & 0 & 0 & 0 & 0 & 0 & 0 & 0 & 0 & 0 & 0 & 0 & 0 \\ 0 & 1 & 1 & 0 & 1 & 1 & 0 & 0 & 0 & 0 & 0 & 0 & 0 & 0 & 0 & 0 & 0 & 0 & 1 & 0 \\ 0 & 0 & 1 & 1 & 0 & 1 & 1 & 0 & 0 & 0 & 1 & 0 & 0 & 0 & 0 & 0 & 0 & 0 & 0 & 0 \\ 0 & 0 & 0 & 1 & 1 & 0 & 0 & 1 & 0 & 1 & 0 & 0 & 0 & 0 & 0 & 0 & 0 & 0 & 0 & 0 \\ 0 & 0 & 0 & 0 & 1 & 0 & 0 & 1 & 1 & 0 & 0 & 0 & 0 & 0 & 0 & 0 & 0 & 0 & 0 & 0 \\ 0 & 0 & 0 & 0 & 0 & 1 & 1 & 0 & 1 & 1 & 0 & 0 & 0 & 0 & 0 & 0 & 0 & 0 & 0 & 0 \\ 0 & 0 & 0 & 0 & 0 & 0 & 1 & 1 & 0 & 0 & 1 & 0 & 0 & 0 & 0 & 1 & 0 & 0 & 0 & 0 \\ 0 & 0 & 0 & 0 & 0 & 1 & 0 & 1 & 0 & 0 & 1 & 1 & 0 & 0 & 0 & 0 & 0 & 0 & 0 & 0 \\ 0 & 0 & 0 & 0 & 1 & 0 & 0 & 0 & 1 & 1 & 0 & 0 & 1 & 0 & 0 & 0 & 0 & 0 & 0 & 0 \\ 0 & 0 & 0 & 0 & 0 & 0 & 0 & 0 & 0 & 1 & 0 & 0 & 1 & 1 & 0 & 0 & 0 & 1 & 0 & 0 \\ 0 & 0 & 0 & 0 & 0 & 0 & 0 & 0 & 0 & 0 & 1 & 1 & 0 & 1 & 1 & 0 & 0 & 0 & 0 & 0 \\ 0 & 0 & 0 & 0 & 0 & 0 & 0 & 0 & 0 & 0 & 0 & 1 & 1 & 0 & 1 & 1 & 0 & 0 & 0 & 0 \\ 0 & 0 & 0 & 0 & 0 & 0 & 0 & 0 & 0 & 0 & 0 & 0 & 1 & 1 & 0 & 1 & 1 & 0 & 0 & 0 \\ 0 & 0 & 0 & 0 & 0 & 0 & 0 & 0 & 1 & 0 & 0 & 0 & 0 & 1 & 1 & 0 & 1 & 1 & 0 & 0 \\ 0 & 0 & 0 & 0 & 0 & 0 & 0 & 0 & 0 & 0 & 0 & 0 & 0 & 0 & 1 & 1 & 0 & 1 & 1 & 0 \\ 0 & 0 & 0 & 0 & 0 & 0 & 0 & 0 & 0 & 0 & 0 & 1 & 0 & 0 & 0 & 1 & 1 & 0 & 0 & 1 \\ 1 & 0 & 0 & 1 & 0 & 0 & 0 & 0 & 0 & 0 & 0 & 0 & 0 & 0 & 0 & 0 & 1 & 0 & 0 & 0 \\ 1 & 1 & 0 & 0 & 0 & 0 & 0 & 0 & 0 & 0 & 0 & 0 & 0 & 0 & 0 & 0 & 0 & 1 & 0 & 0\end{array}$


Table A8: Adjacency matrix of the centralized network

$\begin{array}{llllllllllllllllllll}0 & 1 & 1 & 1 & 1 & 1 & 1 & 1 & 1 & 1 & 1 & 1 & 1 & 1 & 1 & 0 & 1 & 0 & 0 & 1 \\ 1 & 0 & 1 & 1 & 0 & 0 & 0 & 0 & 0 & 0 & 0 & 0 & 1 & 1 & 0 & 0 & 0 & 0 & 0 & 0 \\ 1 & 1 & 0 & 0 & 1 & 1 & 1 & 0 & 0 & 1 & 0 & 0 & 0 & 0 & 0 & 0 & 0 & 0 & 0 & 1 \\ 1 & 1 & 0 & 0 & 0 & 0 & 0 & 0 & 0 & 0 & 0 & 0 & 0 & 0 & 0 & 0 & 0 & 0 & 1 & 0 \\ 1 & 0 & 1 & 0 & 0 & 0 & 0 & 0 & 1 & 0 & 0 & 0 & 0 & 0 & 0 & 0 & 0 & 0 & 0 & 0 \\ 1 & 0 & 1 & 0 & 0 & 0 & 0 & 0 & 0 & 0 & 0 & 0 & 0 & 0 & 0 & 0 & 0 & 1 & 0 & 0 \\ 1 & 0 & 1 & 0 & 0 & 0 & 0 & 1 & 0 & 0 & 1 & 0 & 0 & 0 & 1 & 1 & 0 & 0 & 1 & 0 \\ 1 & 0 & 0 & 0 & 0 & 0 & 1 & 0 & 0 & 0 & 0 & 1 & 0 & 0 & 0 & 0 & 0 & 0 & 0 & 0 \\ 1 & 0 & 0 & 0 & 1 & 0 & 0 & 0 & 0 & 0 & 0 & 0 & 0 & 0 & 0 & 0 & 1 & 0 & 0 & 0 \\ 1 & 0 & 1 & 0 & 0 & 0 & 0 & 0 & 0 & 0 & 0 & 0 & 0 & 0 & 0 & 1 & 0 & 0 & 0 & 0 \\ 1 & 0 & 0 & 0 & 0 & 0 & 1 & 0 & 0 & 0 & 0 & 0 & 0 & 0 & 0 & 0 & 0 & 0 & 0 & 0 \\ 1 & 0 & 0 & 0 & 0 & 0 & 0 & 1 & 0 & 0 & 0 & 0 & 0 & 0 & 0 & 0 & 0 & 0 & 0 & 0 \\ 1 & 1 & 0 & 0 & 0 & 0 & 0 & 0 & 0 & 0 & 0 & 0 & 0 & 0 & 0 & 0 & 0 & 0 & 0 & 0 \\ 1 & 1 & 0 & 0 & 0 & 0 & 0 & 0 & 0 & 0 & 0 & 0 & 0 & 0 & 0 & 0 & 0 & 0 & 0 & 0 \\ 1 & 0 & 0 & 0 & 0 & 0 & 1 & 0 & 0 & 0 & 0 & 0 & 0 & 0 & 0 & 0 & 0 & 0 & 0 & 0 \\ 0 & 0 & 0 & 0 & 0 & 0 & 1 & 0 & 0 & 1 & 0 & 0 & 0 & 0 & 0 & 0 & 0 & 1 & 0 & 0 \\ 1 & 0 & 0 & 0 & 0 & 0 & 0 & 0 & 1 & 0 & 0 & 0 & 0 & 0 & 0 & 0 & 0 & 0 & 0 & 0 \\ 0 & 0 & 0 & 0 & 0 & 1 & 0 & 0 & 0 & 0 & 0 & 0 & 0 & 0 & 0 & 1 & 0 & 0 & 0 & 0 \\ 0 & 0 & 0 & 1 & 0 & 0 & 1 & 0 & 0 & 0 & 0 & 0 & 0 & 0 & 0 & 0 & 0 & 0 & 0 & 0 \\ 1 & 0 & 1 & 0 & 0 & 0 & 0 & 0 & 0 & 0 & 0 & 0 & 0 & 0 & 0 & 0 & 0 & 0 & 0 & 0\end{array}$


Figure A8: Instructions of the experiment in English

\section{Instructions}

These are the instructions for the experiment. These instructions are the same as on the screen. The instructions will remain available during the experiment. Please read the instructions carefully.

This experiment is divided into three main parts and an introduction part. In the introduction part three practice rounds will be played. During the three main parts of the experiment 20 rounds will be played. Before each part, you will be paired randomly and anonymously with 1 or more participants. The participants you are paired with will remain the same during the rounds of the different parts. So, in the main rounds the participants you are paired with will be the same for 20 rounds. After one part ends you will be randomly matched again. After the second random matching you probably play against other participants, but there is a chance they can also be the same.

Before each part you will be randomly designated a type. Your type can be Blue or Yellow. Prior to the start of the part you will learn which type you are. You will remain the same type during the rounds of the different parts. But before each part you will be randomly assigned a type again. It will be clearly stated when a part ends and you will be assignment a new type and become randomly paired to new participants again.

Each of you must independently and simultaneously make a decision between 'blue' and 'yellow'. Your payoffs will be determined by the choices of the players you are paired with and the type you are. Your type, Blue or Yellow, will indicate how many points you can earn with the decision 'blue' or 'yellow'. Below you find 2 tables, the table on the left for type Blue and the table on the right for type Yellow. In case you play against 1 other participant, when your type is Blue (left table), you choose 'blue' and the other participant also chooses 'blue', the payoff for that round is 10. When your type is Blue and you choose 'yellow' and the other participant also chooses 'yellow', the payoff for that round is 8 . When you choose 'blue' and the other participant chooses 'yellow' or the other way around, the payoff will be 0 . When your type is Yellow (table on the right) you get the highest payoff of 10 , when you and the other participant both choose yellow. 8 points when you both choose blue. And 0 point when you and the other participant don't choose the same colour.

Figure 1: Overview of possible profit points

\begin{tabular}{|l|l|c|c|}
\hline \multicolumn{2}{|l|}{ Your type: Blue } & \multicolumn{2}{l|}{ Your choice } \\
\cline { 3 - 4 } \multicolumn{2}{|c|}{} & Blue & Yellow \\
\hline $\begin{array}{l}\text { Other } \\
\text { participant's } \\
\text { choice }\end{array}$ & Blue & 10 & 0 \\
\cline { 2 - 4 } & Yellow & 0 & 8 \\
\hline
\end{tabular}

\begin{tabular}{|l|l|c|c|}
\hline \multicolumn{2}{|l|}{ Your type: Yellow } & \multicolumn{2}{l|}{ Your choice } \\
\cline { 3 - 4 } \multicolumn{2}{|l|}{} & Blue & Yellow \\
\hline $\begin{array}{l}\text { Other } \\
\text { participant's } \\
\text { choice }\end{array}$ & Blue & 8 & 0 \\
\cline { 2 - 4 } & Yellow & 0 & 10 \\
\hline
\end{tabular}

Note that your type will remain the same during the rounds of a part of the experiment, but you can change your decision every round. In this experiment you are randomly paired with 1 or more players who may or may not have the same type as you have. You will not be able to see which type the others are. You make one decision that holds for all participants you are paired with. The payoff of a round will be the average of the payoffs you get from the participants you are paired with. Thus, if you are paired with two other participants and you payoff with one would be 10 according to the table above and the payoff with the other would be 0 , you earn 5 points in that round. At the end of the experiment, the points you earned will be paid out to you at an exchange rate of 50 points per euro. 
Figure A9: Instructions of the experiment in Spanish

\section{Instrucciones}

Estas son las instrucciones para el experimento. Estas instrucciones son las mismas que en la pantalla. Las instrucciones permanecerán disponibles durante el experimento. Por favor, lea atentamente las instrucciones.

Este experimento se divide en tres partes principales y una parte de introducción. En la parte de introducción se jugarán tres rondas de práctica. Durante las tres partes principales del experimento se jugarán 20 rondas. Antes de cada parte, se le emparejará de forma aleatoria y anónima con uno o más participantes. Los participantes con los que estás emparejado seguirán siendo los mismos durante las rondas de las diferentes partes. Por lo tanto, en las rondas principales, los participantes con los que estés emparejado serán los mismos durante 20 rondas. Después de que una parte termina usted será emparejado al azar otra vez. Después de la segunda coincidencia aleatoria, es probable que juegues contra otros participantes, pero existe la posibilidad de que también sean iguales.

Antes de cada parte se le designará un tipo al azar. Tu tipo puede ser azul o amarillo. Antes del comienzo de la parte usted aprenderá qué tipo usted es. Usted seguirá siendo del mismo tipo durante las rondas de las diferentes partes. Pero antes de cada parte se le asignará un tipo al azar de nuevo. Se indicará claramente cuando termine una parte y se le asignará un nuevo tipo y se le emparejará de nuevo al azar con nuevos participantes.

Cada uno de ustedes debe tomar una decisión de forma independiente y simultánea entre "azul" y "amarillo". Tus ganancias serán determinadas por las elecciones de los jugadores con los que estás emparejado y el tipo de jugador que eres. Tu tipo, Azul o Amarillo, te indicará cuántos puntos puedes ganar con la decisión "azul" o "amarillo". Debajo encontrará 2 tablas, la tabla de la izquierda para el tipo Azul y la tabla de la derecha para el tipo Amarillo. Durante los emparejamientos, cuando tu tipo es Azul (tabla de la izquierda), y eliges 'azul' y el otro participante también elige 'azul', el resultado de esa ronda es de 10 puntos, en cambio si ambos elegís 'amarillo' el resultado será de 8 puntos. De la misma forma, cuando tu tipo es Amarillo (tabla de la derecha) obtienes la máxima puntuación de 10 cuando ambos escogéis 'amarillo' y 8 cuando ambos elegís 'azul'. Si tú y el otro participante no escogéis el mismo color el resultado son 0 puntos.

Figura 1: Resumen de los posibles puntos de beneficio

\begin{tabular}{|l|l|l|l|}
\hline \multicolumn{2}{|l|}{ Tu tipo: Azul } & \multicolumn{2}{l|}{ Tu elección } \\
\cline { 3 - 4 } \multicolumn{2}{|l|}{} & Azul & Amarillo \\
\hline $\begin{array}{l}\text { Elección de } \\
\text { otro } \\
\text { participante }\end{array}$ & Azul & 10 & 0 \\
\cline { 2 - 4 } & Amarillo & 0 & 8 \\
\hline
\end{tabular}

\begin{tabular}{|l|l|l|l|}
\hline \multicolumn{2}{|l|}{ Tu tipo: Amarillo } & \multicolumn{2}{|l|}{ Tu elección } \\
\cline { 3 - 4 } \multicolumn{2}{|l|}{} & Azul & Amarillo \\
\hline $\begin{array}{l}\text { Elección de } \\
\text { otro } \\
\text { participante }\end{array}$ & Azul & 8 & 0 \\
\cline { 2 - 4 } & Amarillo & 0 & 10 \\
\hline
\end{tabular}

Tenga en cuenta que su tipo seguirá siendo el mismo durante las rondas de una parte del experimento, pero puede cambiar su decisión en cada ronda. En este experimento usted es emparejado aleatoriamente con 1 o más jugadores que pueden o no tener el mismo tipo que usted tiene. No podrá ver de qué tipo son los otros. Tu elección en cada ronda se utilizará para resolver todos tus emparejamientos de esa ronda. El pago de cada ronda será el promedio de las recompensas obtenidas con todos tus emparejamientos. Por ejemplo, si usted está emparejado con otros dos participantes y en uno de los casos su recompensa es 10 puntos y en el otro es 0 su ganancia de esta ronda serian 5 puntos. Al final del experimento, los puntos ganados se le pagarán a un tipo de cambio de 50 puntos por euro. 
Figure A10: Example computational and experimental results random network. First round: Left computational model. Right: Experimental results
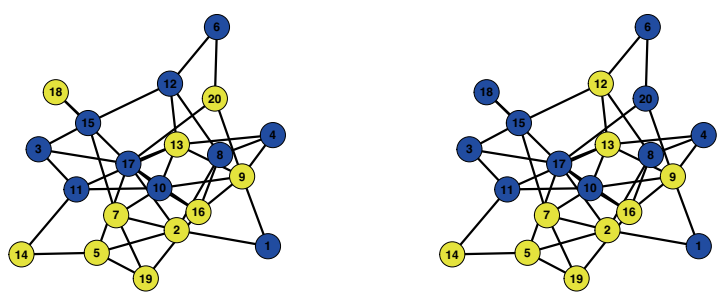

Figure A11: Example computational and experimental results random network. Last round: Left computational model. Right: Experimental results
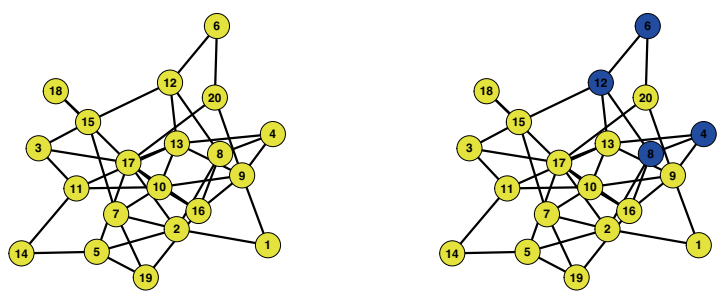
Figure A12: Example computational and experimental results small world network. First round: Left computational model. Right: Experimental results
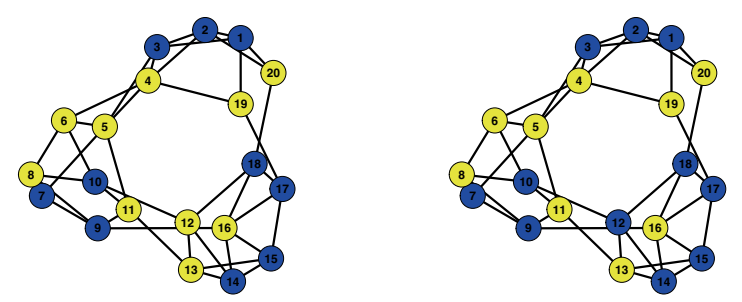

Figure A13: Example computational and experimental results small world network. Last round: Left computational model. Right: Experimental results
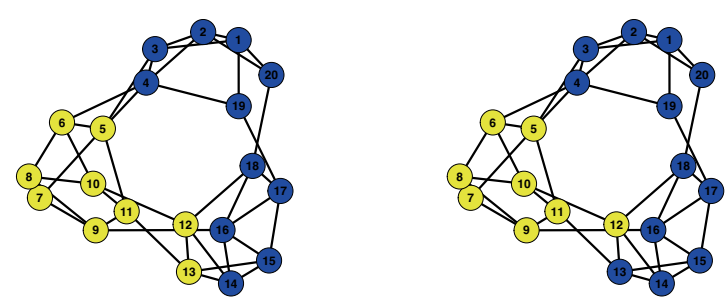
Figure A14: Example computational and experimental results Network with central node network. First round: Left computational model. Right: Experimental results
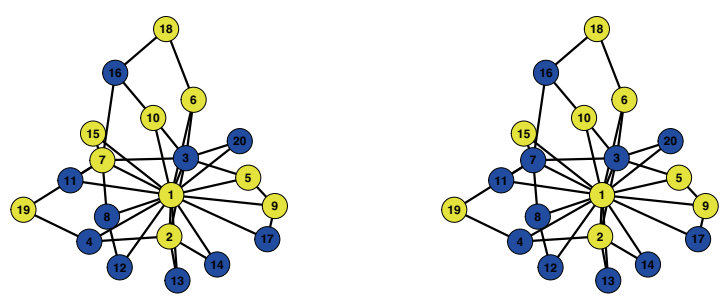

Figure A15: Example computational and experimental results Network with central node network. Last round: Left computational model. Right: Experimental results
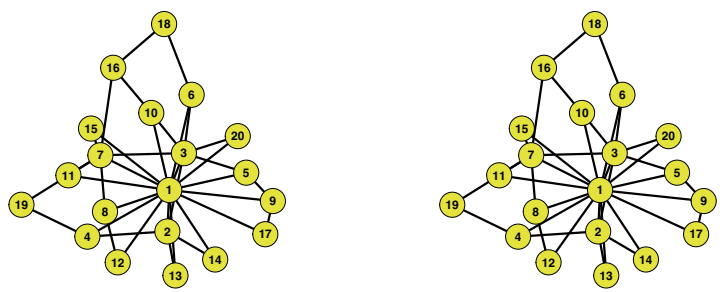


\section{Appendices Chapter 5}

Table A9: Logistic regression with dependent variable generalized trust with method by of Tacchella et al., (2012)

\begin{tabular}{lcc}
\hline \hline & $(1)$ & $(2)$ \\
\hline Occupational complexity & $0.057^{* * *}(0.006)$ & $0.033^{* * *}(0.006)$ \\
Age & $0.033^{* * *}(0.003)$ \\
College educated & $1.423^{* * *}(0.100)$ \\
School dropout & $-1.324^{* * *}(0.188)$ \\
Income & $0.124^{* * *}(0.026)$ \\
Male & $0.590^{* * *}(0.091)$ \\
Town & $-0.312(0.217)$ \\
Small city & $-12.033^{* * *}(1.151)$ & $-10.526^{* * *}(1.329)$ \\
bigcity1 & 2,867 & 2,867 \\
Constant & $-1,820.621$ & $-1,496.204$ \\
\hline Observations & $3,645.242$ & $3,012.408$ \\
Log Likelihood & ${ }^{*} p<0.1 ;{ }^{* *} p<0.05 ;{ }^{* * *} p<0.01$ \\
Akaike Inf. Crit. & & $-0.585^{* *}(0.246)$ \\
\hline \hline Note: & &
\end{tabular}




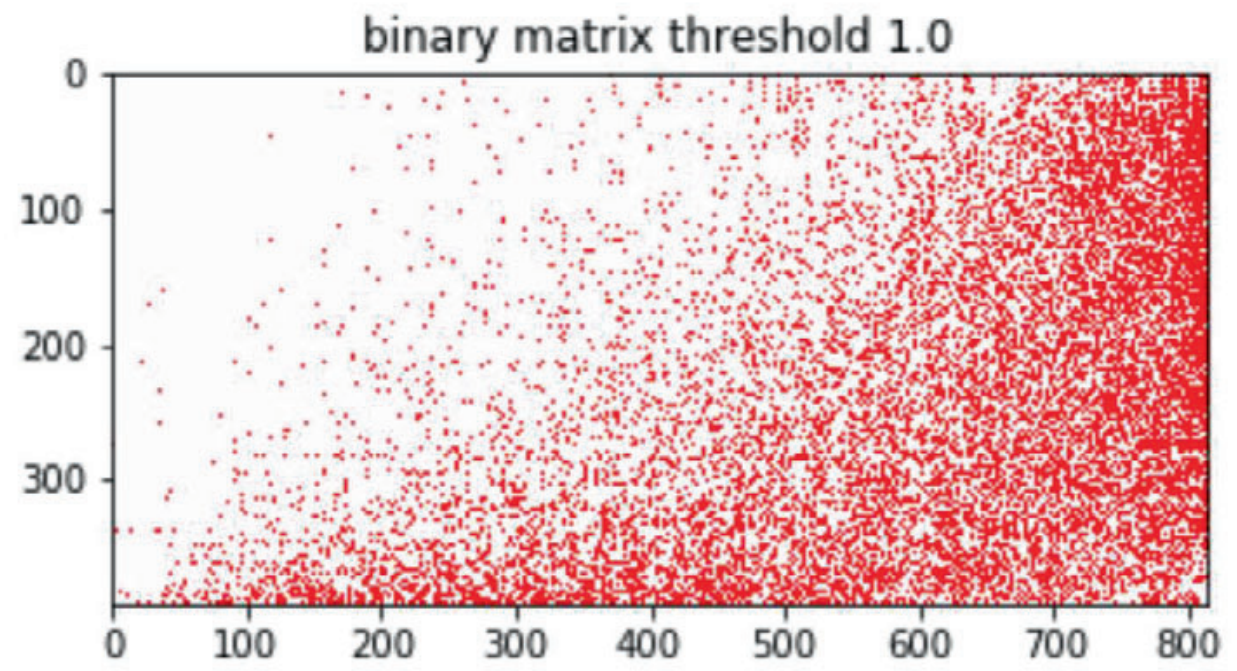

Figure A16: City-occupation matrix. On the y-axis the $400 \mathrm{MSAs}$, on the $\mathrm{x}$-axis the 840 occupations. The cell is red when an occupation has a RCA $>1$ in that MSA. The matrix is ordered by number of occupations in a MSA, where the MSA with the lowest number of occupations is on top and the MSA with the highest number of occupations is at he bottom. From left to right the matrix is order by the amount of times an occupation occurs in an MSA. Left the occupations that occur the least and right the occupations that occur the most. 
Table A10: Logistic regression with dependent variable generalized trust, for the cutoff value $R C A=1.5$

\begin{tabular}{lcc}
\hline \hline & $(1)$ & $(2)$ \\
\hline complcl & $0.030^{* * *}(0.003)$ & $0.018^{* * *}(0.003)$ \\
age & $0.034^{* * *}(0.003)$ \\
co_educ1 & $1.393^{* * *}(0.100)$ \\
dropout1 & $-1.368^{* * *}(0.190)$ \\
income & $0.107^{* * *}(0.026)$ \\
male1 & & $0.569^{* * *}(0.091)$ \\
town1 & & $-0.453^{* *}(0.215)$ \\
smallcity1 & $-1.067^{* * *}(0.242)$ \\
bigcity1 & & $-0.963^{* * *}(0.246)$ \\
Constant & & $-3.993^{* * *}(0.397)$ \\
\hline Observations & 2,867 & 2,867 \\
Log Likelihood & $-1,811.438$ & $-1,494.384$ \\
Akaike Inf. Crit. & $3,626.875$ & $3,008.769$ \\
\hline \hline Note: & \multirow{2}{*}{$\mathrm{p}<0.1 ;{ }^{* *} \mathrm{p}<0.05 ; * * * \mathrm{p}<0.01$}
\end{tabular}


Nederlandse Samenvatting 



\section{Nederlandse Samenvatting}

Netwerk structuur wordt vaak als oplossing gezien voor coöperatie problemen en sociale dilemma's. In het eerste deel van deze dissertatie wordt het effect van netwerk structuur op sociaal dilemma genaamd asymmetrische coördinatie bestudeerd. In de hoofstukken 2, 3 en 4 wordt het effect van netwerk eigenschappen op deze coördinatie processen bestudeerd middels drie verschillende methoden. In hoofdstuk 2 wordt een simulatie model gecreëerd. In hoofdstuk 3 worden de voorspellingen die gedaan worden door het simulatie model uit hoofdstuk 2 getest in een laboratorium experiment met 240 mensen. In hoofdstuk 4 proberen we de resultaten verder te generaliseren door dezelfde asymmetrische coördinatie processen te modeleren met methoden uit de statistische fysica. In het tweede deel van deze dissertatie wordt de vraag gesteld hoe algemeen vertrouwen kan ontstaan in een samenleving die steeds individualistischer wordt. In hoofdstuk 5 beargumenteren we dat een oude theorie genaamd "organische solidariteit" een belangrijke rol kan spelen bij het beantwoorden van deze vraag. De theorie stelt dat verdeling van arbeid één van de belangrijkste fundamenten is voor menselijke coöperatie. In dit hoofdstuk bestuderen we aspecten van de arbeidsverdeling en laten we zien dat deze sterk gecorreleerd zijn met algemeen vertrouwen.

\section{Hoofdstuk 2}

In de hoofdstukken 2, 3 en 4 staat een asymmetrisch coördinatie spel genaamd "battle of the sexes" centraal. Het spel weergegeven in Table A11 schetst een situatie met twee spelers. De rij speler en de kolom speler. Beide spelers kunnen een keuze maken tussen twee opties, namelijk $\alpha$ en $\beta$. De twee keuze combinaties vormen vier mogelijke uitkomsten, weergegeven in de matrix, waarbij het eerste cijfer de gelukwaarde van de rijspeler betreft en het tweede cijfer de gelukwaarde voor de kolom speler. Beide spelers hebben de hoogste gelukwaarde als ze hetzelfde kiezen, echter de rij speler heeft een hogere gelukwaarde als beide spelers kiezen voor $\alpha$ en de kolom speler heeft een hogere gelukwaarde als beide spelers kiezen voor $\beta$.

Table A11: Tabel voor een asymmetrisch coördinatie spel

\begin{tabular}{c|cc} 
& $\alpha$ & $\beta$ \\
\hline$\alpha$ & 2,1 & 0,0 \\
$\beta$ & 0,0 & 1,2
\end{tabular}


Table A12: Tabel voor een asymmetrisch coördinatie spel met drie speleres

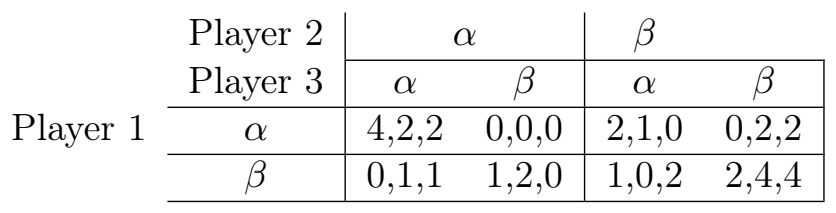

Het sociale dilemma is dat beide spelers willen coördineren op dezelfde keuze, maar dat ze beide een voorkeur hebben om te coördineren op een andere optie. In een experimentele laboratorium setting waarin mensen dit spel moeten spelen en de resulterende gelukwaarde uitbetaalt krijgen, komt het meest voor dat het niet lukt om hun keuzes te coördineren (Cooper et al., 1989; Binmore et al., 2007). Dit komt omdat beide spelers vaak hun "favoriete" optie kiezen. Als dezelfde twee spelers de kans krijgen om het spel vaker dan één keer te spelen, dan lukt het vaak wel om tot coördinatie te komen, echter is het vooraf niet duidelijk op welke van de twee evenwichten de spelers zullen eindigen. In enkele gevallen zullen de spelers zelfs tegelijk wisselen tussen allebei $\alpha$ en allebei $\beta$ op het moment dat het spel meerdere keren gespeeld wordt (Duffy et al., 2017; De Kwaadsteniet et al., 2012; Lau and Mui, 2008). Dit gedrag is het meest kosten efficiënt.

In Tabel A12 is dezelfde situatie geschetst met 2 keuze opties maar dan voor drie spelers. In de drie speler situatie is het al makkelijker te voorspellen wat er gekozen gaat worden, omdat het spel niet meer in balans is. Intuïtief wordt dit duidelijk doordat als er drie mensen moeten kiezen tussen twee opties, er altijd een meerderheid voor één van de twee opties is. In hoofdstuk 2 willen we weten wat er gebeurd als je dit $2 \times 2$ spel speelt waarbij de spelers interacter middels een netwerk. In het netwerk zijn dan de speler de knopen (nodes) en ze interacteren met de andere knopen met wie ze verbonden zijn middels een lijn (edges). De intuïtie is dat de structuur van het netwerk invloed zal hebben op de uiteindelijke keuze van de spelers omdat er vaak een lokale meerderheid is voor één van de twee opties, zoals te zien in Tabel A12. De structuur van het netwerk kan daarom van cruciaal belang zijn om het keuze gedrag in (sociale) netwerken te begrijpen.

In hoofdstuk 2 maken we een simulatie model voor deze asymmetrische keuze processen op netwerken. We simuleren de coordinatie processen voor drie type netwerken en analyseren de patronen voor deze netwerken. We concluderen dat de patronen sterk afhangen van het type netwerk. De patronen zijn het minst sterk in random 'Erdös-Rényi' netwerken. Het gedrag laat zich hier het minst 
goed voorspellen omdat er weinig structuur zit in de netwerken. In geclusterde 'small world' netwerken zien we homogeen gedrag (dezelfde keuzes) binnen de clusters van het netwerk, maar heterogeen (verschillende keuzes) tussen clusters. Het gedrag laat zich al makkelijker voorspellen in dit type netwerk. In een derde type netwerk, het gecentraliseerde netwerk, wordt het gedrag voor een groot deel gedetermineerd door de meest centrale knoop. In dit type netwerk kan het gedrag makkelijk voorspelt worden.

\section{Hoofdstuk 3}

In hoofdstuk 3 gebruiken we de resultaten van hoofdstuk 2 en testen deze in een laboratorium experiment met 240 menselijke subjecten. De menselijke subjecten spelen asymmetrische coördinatie spellen in groepen van 20. Met wie de subjecten interacteren is afhankelijk van hun plaats in het netwerk. De subjecten spelen de spellen in drie verschillende netwerken die de netwerken van hoofdstuk 2 representeren. We onderzoeken of de simulatie resultaten van hoofdstuk 2 samenhangen met de keuzes van de menselijke subjecten en dus hoe deze afhankelijk zijn van het netwerk waarin de spelers zich bevinden. In lijn der verwachting laten de menselijke keuzes zich moeilijk voorspellen in het random 'Erdös-Rényi' netwerk. In het geclusterde 'small world' netwerk zijn de keuzes van de subjecten al meer in lijn met de voorspellingen van het simulatie model. Tevens vinden we ook hier homogeen gedrag binnen de clusters van het netwerk, maar heterogeen (verschillende keuzes) tussen clusters. In het gecentraliseerde netwerk zijn de keuzes van het simulatie model bijna geheel hetzelfde aan de keuzes van de menselijke subjecten, omdat het gedrag vrijwel geheel gedetermineerd wordt door de meest centrale speler.

\section{Hoofdstuk 4}

In hoofdstuk 4 proberen we de resultaten van de voorgaande hoofdstukken verder te generaliseren door de evenwichten uit het asymetrische spel analytisch te berekenen. Om de analysische uitkomsten te verkrijgen maken we gebruik van het ferromagnetische Ising model. We generaliseren het ferromagnetische Ising model zodat deze ook de asymmetrische voorkeuren kan representeren. De asymmetrische interacties worden gerealiseerd door de parameter die in het oorspronkelijke model het 'extern magnetisch veld' vertegenwoordigd, te laten variëren tussen de knooppunten. De 'externe' magnetisatie kan in dit geval dus anders zijn voor de ene knoop ten opzichte van de andere knoop. De asymmetrische voorkeuren kunnen dus 
gemodelleerd worden door middel van de magnetisatie van de individuele knooppunten. De resultaten laten een verschil zien in de gevonden evenwichten tussen het simulatiemodel en het Ising-model. Met het Ising-model vinden we een rijkere set van evenwichten in vergelijking tot het simulatiemodel. Deze bevinding versterkt eigenlijk ons vertrouwen in de resultaten van het simulatiemodel, omdat deze resultaten lijken te suggereren dat als we het coördinatieprobleem in hoofdstuk 2 analytisch modelleren, de resultaten sterker worden. Een vermoedelijke reden dat de resultaten sterker worden wanneer ze analytisch gemodelleerd worden, is dat de update-regel die gebruikt wordt in het simulatiemodel waarschijnlijk geen instabiele evenwichtspunten vindt. Er moet echter worden opgemerkt dat we niet in staat waren om het Ising-model zo te formuleren dat het nauwkeurig overeenkomt met de resultaten van het simulatiemodel in alle onderzochte netwerken.

\section{Hoofdstuk 5}

In hoofdstuk 5 erkennen we dat modellen van kleine groepen met herhaalde interacties de sociale cohesie niet volledig kunnen verklaren. Moderne samenlevingen functioneren niet alleen op basis van herhaalde interacties met bekenden. Hoe complexer een samenleving wordt, hoe meer het dagelijks leven wordt bepaald door sociale interacties met mensen die men niet kent. Mensen interacteren dagelijks met mensen die ze nog niet eerder hebben ontmoet. Herhaalde interacties en de structuur van het sociale netwerk kunnen dus wel enige variatie in het cohesieprobleem verklaren, maar niet alles. In hoofdstuk 5 stellen we dat sociale interacties voor een belangrijk deel gevormd worden door arbeidsdeling en regelgevende instanties. Hierbij gebruiken we een klassieke sociologische theorie genaamd 'organische solidariteit'. De theorie is lange tijd afwezig geweest in sociologische discussies, maar is recent weer geintroduceerd.

Fenomenen als sociale cohesie, samenwerking en vertrouwen kunnen zeker deels worden begrepen via de herhaalde interacties van kleine groepen. Maar in moderne samenlevingen zijn deze interacties echter ingebed in een grotere maatschappelijke structuur. De 'grotere maatschappelijke structuur' kan hier begrepen worden als het geheel van formele en informele instituten. 'Instituten' kan hier opgevat worden in de breedst mogelijke zin, gedefinieerd als 'stabiele, terugkerende gedragspatronen'. De gedragspatronen kunnen formeel geordend en afgedwongen worden, zoals wetten en handhaving, of zelfregulerend en informeel, zoals verschillende vormen van cultuur.

We stellen dat het effect van de arbeidsdeling, hoewel een instituut op zich, on- 
afhankelijk van andere soorten instituten moet worden bestudeerd. Zo veroorzaakt iemands werk een krachtige (economische) inbedding die mensen dwingt om zich dagelijks met anderen te verhouden. Als een klant bijvoorbeeld een winkel binnenkomt, zijn de maatschappelijke rollen direct duidelijk. Zowel de winkeleigenaar als de klant moet een bepaalde maatschappelijke standaard handhaven om zijn economische positie te behouden. Daarmee helpen economische relaties de maatschappelijke orde te bepalen. Hoe deze relaties zijn gestructureerd kan dus van vitaal belang zijn voor verschillende sociale mechanismen die verder reiken dan het economische domein.

In een recente studie, geïnspireerd door Durkheim's theorie over organische solidariteit, theoretiseren auteurs over de relevantie van deze theorie in het huidige sociologische debat (Portes and Vickstrom, 2011; Durkheim, 1893). Ze schrijven dat organische solidariteit afhankelijk is van drie voorwaarden, namelijk:

1. (Economische) Diversiteit onder de leden van een samenleving

2. Sterke coördinerende instituten

3. Complexe arbeidsdeling (specialisatie)

De invloed van formele instituten op positieve resultaten voor de samenleving, zoals samenwerking en vertrouwen, is een breed bestudeerd onderwerp en onderwerp van een voortdurend debat (Fehr and Gachter, 2000; Kosfeld et al., 2009; Gürerk et al., 2006; Lo Iacono, 2019; IOS, 2019). Het effect van een complexe arbeidsdeling en economische diversiteit is echter nog niet empirisch getest.

In hoofdstuk 5 gebruiken we recente methoden uit het veld van de economische complexiteit die gericht zijn op het kwantificeren van de (economische) diversiteit onder de leden van een samenleving en de specialisatie van een beroep. We gebruiken de verkregen variabelen en toetsen hoe deze zich verhouden tot het zelf-gerapporteerde algemeen vertrouwen. We beargumenteren dat algemeen vertrouwen niet kan worden verklaard door herhaalde interacties tussen individuen. In de gebruikte enquêtevraag die generalized trust meet, wordt de respondent gevraagd: 'Generally speaking, would you say that most people can be trusted or that you can't be too careful in dealing with people?'. De vraag is of mensen in het algemeen te vertrouwen zijn en niet alleen mensen die men kent of met wie men eerder interacties heeft gehad. Om algemeen vertrouwen zullen we daarom gebruik moeten maken van andere verklaringen, zoals organische solidariteit. In 
hoofdstuk 5 laten we zien dat er een groot effect is van zowel beroepsmatige diversiteit als beroepsmatige complexiteit op het het zelfgerapporterede algemeen vertrouwen van de respodent. 

Bibliography 


\section{Bibliography}

Abascal, M. and Baldassarri, D. (2015). Love thy neighbor? ethnoracial diversity and trust reexamined. American Journal of Sociology, 121(3):722-782.

Albert, R. and Barabási, A.-L. (2002). Statistical mechanics of complex networks. Reviews of Modern Physics, 74(1):47.

Allison, S. T. and Kerr, N. L. (1994). Group correspondence biases and the provision of public goods. Journal of Personality and Social Psychology, 66(4):688.

Alonso-Sanz, R. (2011). Self-organization in the battle of the sexes. International Journal of Modern Physics C, 22(01):1-11.

Alonso-Sanz, R. (2012a). A quantum battle of the sexes cellular automaton. In Proc. R. Soc. A, page rspa20120161. The Royal Society.

Alonso-Sanz, R. (2012b). The spatialized, continuous-valued battle of the sexes. Dynamic Games and Applications, 2(2):177-194.

Amaral, M. A., Wardil, L., Perc, M., and da Silva, J. K. (2016). Evolutionary mixed games in structured populations: Cooperation and the benefits of heterogeneity. Physical Review E, 93(4):042304.

Antonioni, A., Sanchez, A., and Tomassini, M. (2014). Global information and mobility support coordination among humans. Scientific Reports, 4:6458.

Axelrod, R. and Dion, D. (1988). The further evolution of cooperation. Science, 242(4884):1385-1390.

Axelrod, R. and Hamilton, W. D. (1981). The evolution of cooperation. Science, 211(4489):1390-1396.

Balland, P. A. (2017). Economic geography in r: Introduction to the econgeo package.

Barabási, A.-L. and Albert, R. (1999). Emergence of scaling in random networks. Science, 286(5439):509-512.

Baxi, U. (1974). Comment-durkheim and legal evolution: some problems of disproof. Law \& Society Review, 8(4):645-651.

Becker, G. S. and Murphy, K. M. (1992). The division of labor, coordination costs, and knowledge. The Quarterly Journal of Economics, 107(4):1137-1160. 
Becker, T. E. (2005). Potential problems in the statistical control of variables in organizational research: A qualitative analysis with recommendations. Organizational Research Methods, 8(3):274-289.

Bettencourt, L. M. A., Samaniego, H., and Youn, H. (2014). Professional diversity and the productivity of cities. Scientific Reports, 4:3-8.

Binmore, K. et al. (2007). Playing for real: a text on game theory. Oxford university press.

Binmore, K. G. (1994). Game theory and the social contract: just playing, volume 2. MIT press.

BLS (2019). Bureau of labor statistics. https://www.bls.gov/sae/data/home.htm. Accessed: 2019-11-23.

Bojanowski, M. and Buskens, V. (2011). Coordination in dynamic social networks under heterogeneity. The Journal of Mathematical Sociology, 35(4):249-286.

Borsboom, D. and Cramer, A. O. (2013). Network analysis: an integrative approach to the structure of psychopathology. Annual review of clinical psychology, 9:91-121.

Broere, J., Buskens, V., Stoof, H., and Sánchez, A. (2019). An experimental study of network effects on coordination in asymmetric games. Scientific Reports, $9(1): 6842$.

Broere, J., Buskens, V., Weesie, J., and Stoof, H. (2017). Network effects on coordination in asymmetric games. Scientific Reports, 7:17016.

Bruggeman, J., Sprik, R., and Quax, R. (2018). Cooperation for public goods driven by noise. arXiv preprint arXiv:1812.0536\%.

Buskens, V. and Raub, W. (2002). Embedded trust: Control and learning. Advances in Group Processes, 19(2002):167-202.

Buskens, V. and Raub, W. (2013). Rational choice research on social dilemmas: embeddedness effects on trust. Handbook of rational choice social research, pages $113-150$.

Buskens, V. and Snijders, C. (2015). Effects of network characteristics on reaching the payoff-dominant equilibrium in coordination games: a simulation study. Dynamic Games and Applications, pages 1-18. 
Carroll, S. (2017). The big picture: on the origins of life, meaning, and the universe itself. Penguin.

Chen, D. L., Schonger, M., and Wickens, C. (2016). oTree-an open-source platform for laboratory, online, and field experiments. Journal of Behavioral and Experimental Finance, 9:88-97.

Christman, J. (2017). Social and political philosophy: A contemporary introduction. Routledge.

Cimini, G. and Sánchez, A. (2014). Learning dynamics explains human behaviour in prisoner's dilemma on networks. Journal of The Royal Society Interface, 11(94):20131186.

Coleman, J. S. (1994). Foundations of social theory. Harvard university press.

Cooper, R., DeJong, D. V., Forsythe, R., and Ross, T. W. (1989). Communication in the battle of the sexes game: some experimental results. The RAND Journal of Economics, pages 568-587.

Copeland, D. C. (1996). Economic interdependence and war: A theory of trade expectations. International Security, 20(4):5-41.

Correia, A. and Stoof, H. (2019). Nash equilibria in the response strategy of correlated games. Scientific Reports, 9(1):2352.

Cournot, A. A. (1897). Researches into the Mathematical Principles of the Theory of Wealth. Macmillan.

Crawford, V. P., Gneezy, U., and Rottenstreich, Y. (2008). The power of focal points is limited: Even minute payoff asymmetry may yield large coordination failures. American Economic Review, 98(4):1443-58.

Davidson, D. (1980). Mental events. Readings in philosophy of psychology, 1:107119.

De Kwaadsteniet, E. W., Homan, A. C., Van Dijk, E., and Van Beest, I. (2012). Social information as a cue for tacit coordination. Group Processes 6 Intergroup Relations, 15(2):257-271.

Dekker, M., Walentek, D., Haslbeck, J., and Broere, J. (2020). A general framework for international conflict under incomplete information. Preprint: Apsanet. 
Duffy, J., Lai, E. K., and Lim, W. (2017). Coordination via correlation: an experimental study. Economic Theory, 64(2):265-304.

Durkheim, E. ([1984] 1893). The division of labor in society. Trans. WD Halls. New York, NY: Free Press.

Easley, D. and Kleinberg, J. (2010a). Cascading behavior in networks. Networks, Crowds, and Markets: Reasoning About a Highly Connected World. Cambridge University Press.

Easley, D. and Kleinberg, J. (2010b). Networks, crowds, and markets: Reasoning about a highly connected world. Cambridge University Press.

Enemark, D., McCubbins, M. D., and Weller, N. (2014). Knowledge and networks: An experimental test of how network knowledge affects coordination. Social Networks, 36:122-133.

Evans, C. R. and Dion, K. L. (1991). Group cohesion and performance: A metaanalysis. Small group research, 22(2):175-186.

Eve, R. A., Horsfall, S., and Lee, M. E. (1997). Chaos, complexity, and sociology: Myths, models, and theories. Sage.

Ezaki, T. and Masuda, N. (2017). Reinforcement learning account of network reciprocity. PloS One, 12(12):e0189220.

Fehr, E. and Gachter, S. (2000). Cooperation and punishment in public goods experiments. American Economic Review, 90(4):980-994.

Fortunato, S. (2010). Community detection in graphs. Physics Reports, 486(3$5): 75-174$.

Freeman, L. C. (1977). A set of measures of centrality based on betweenness. Sociometry, pages 35-41.

Galam, S. and Walliser, B. (2010). Ising model versus normal form game. Physica A: Statistical Mechanics and its Applications, 389(3):481-489.

Gartzke, E., Li, Q., and Boehmer, C. (2001). Investing in the peace: Economic interdependence and international conflict. International Organization, $55(2): 391-438$.

Glaeser, E. L., Laibson, D. I., Scheinkman, J. A., and Soutter, C. L. (2000). Measuring trust. The Quarterly Journal of Economics, 115(3):811-846. 
Glauber, R. J. (1963). Time-dependent statistics of the ising model. Journal of Mathematical Physics, 4(2):294-307.

Gracia-Lázaro, C., Ferrer, A., Ruiz, G., Tarancón, A., Cuesta, J. A., Sánchez, A., and Moreno, Y. (2012). Heterogeneous networks do not promote cooperation when humans play a prisoner's dilemma. Proceedings of the National Academy of Sciences, 109(32):12922-12926.

Granovetter, M. and Soong, R. (1983). Threshold models of diffusion and collective behavior. Journal of Mathematical Sociology, 9(3):165-179.

Greiner, B. et al. (2004). The online recruitment system ORSEE 2.0-a guide for the organization of experiments in economics. University of Cologne, Working paper series in economics, 10(23):63-104.

Grujić, J., Fosco, C., Araujo, L., Cuesta, J. A., and Sánchez, A. (2010). Social experiments in the mesoscale: Humans playing a spatial prisoner's dilemma. PloS One, 5(11):e13749.

Gürerk, Ö., Irlenbusch, B., and Rockenbach, B. (2006). The competitive advantage of sanctioning institutions. Science, 312(5770):108-111.

Hardin, G. (1968). The tragedy of the commons. Science, 162(3859):1243-1248.

Haslbeck, J., Epskamp, S., Marsman, M., and Waldorp, L. (2018). Interpreting the ising model: The input matters. arXiv preprint arXiv:1811.02916.

Hauert, C. and Szabó, G. (2005). Game theory and physics. American Journal of Physics, 73(5):405-414.

Hegselmann, R. (2017). Thomas c. schelling and james m. sakoda: The intellectual, technical, and social history of a model. Journal of Artificial Societies and Social Simulation, 20(3).

Hernández, P., Martínez-Cánovas, G., Muñoz-Herrera, M., and Sánchez, A. (2017). Equilibrium characterization of networks under conflicting preferences. Economics Letters, 155:154-156.

Hernandez, P., Muñoz-Herrera, M., and Sánchez, Á. (2013). Heterogeneous network games: Conflicting preferences. Games and Economic Behavior, 79:5666.

Hidalgo, C. and Hausmann, R. (2009). The building blocks of economic complexity. Proceedings of the National Academy of Sciences, 106(26):10570-10575. 
Hobbes, T. (1968). Leviathan; edited with an Introduction by CB Macpherson. Penguin.

Holland, J. H. (2000). Emergence: From chaos to order. OUP Oxford.

Hopfield, J. J. (1982). Neural networks and physical systems with emergent collective computational abilities. Proceedings of the National Academy of Sciences, 79(8):2554-2558.

Huang, K. (2008). Statistical Mechanics, 2nd ed. Wiley India Pvt. Limited.

Hume, D. ([2003] 1739). A treatise of human nature. Courier Corporation.

Huntington, S. P. (2006). Political order in changing societies. Yale University Press.

IBSEN. H2020 project: IBSEN. https://ibsen-h2020.eu. Accessed: 2018-08-10.

IOS (2019). Institutions for open societies. https://www.uu.nl/en/research/institutions-for-open-societies. Accessed: 2019-11-23.

Ising, E. (1925). Beitrag zur theorie des ferromagnetismus. Zeitschrift für Physik A Hadrons and Nuclei, 31(1):253-258.

Jost, L. (2006). Entropy and diversity. Oikos, 113(2):363-375.

Kitsak, M., Gallos, L. K., Havlin, S., Liljeros, F., Muchnik, L., Stanley, H. E., and Makse, H. A. (2010). Identification of influential spreaders in complex networks. Nature Physics, 6(11):888-893.

Kosfeld, M., Okada, A., and Riedl, A. (2009). Institution formation in public goods games. American Economic Review, 99(4):1335-55.

Kramers, H. A. and Wannier, G. H. (1941a). Statistics of the two-dimensional ferromagnet. part i. Physical Review, 60(3):252.

Kramers, H. A. and Wannier, G. H. (1941b). Statistics of the two-dimensional ferromagnet. part ii. Physical Review, 60(3):263.

Krasner, S. D. (1976). State power and the structure of international trade. World Politics, 28(3):317-347.

Ladyman, J., Lambert, J., and Wiesner, K. (2013). What is a complex system? European Journal for Philosophy of Science, 3(1):33-67. 
Lau, S.-H. P. and Mui, V.-L. (2008). Using turn taking to mitigate coordination and conflict problems in the repeated battle of the sexes game. Theory and Decision, 65(2):153-183.

Lo Iacono, S. (2019). Law-breaking, fairness, and generalized trust: The mediating role of trust in institutions. PloS One, 14(8).

Macy, M. W. and Flache, A. (2002). Learning dynamics in social dilemmas. Proceedings of the National Academy of Sciences, 99(suppl 3):7229-7236.

Mäs, M. and Nax, H. H. (2016). A behavioral study of "noise" in coordination games. Journal of Economic Theory, 162:195-208.

Mazzoli, M. and Sanchez, A. (2017). Equilibria, information and frustration in heterogeneous network games with conflicting preferences. Journal of Statistical Mechanics: Theory and Experiment, 2017(11):113403.

McCubbins, M. D., Paturi, R., and Weller, N. (2009). Connected coordination: Network structure and group coordination. American Politics Research, 37(5):899-920.

Mehta, J., Starmer, C., and Sugden, R. (1994). Focal points in pure coordination games: An experimental investigation. Theory and Decision, 36(2):163-185.

Merton, R. K. (1934). Durkheim's division of labor in society. American Journal of Sociology, 40(3):319-328.

Newman, M. E. (2006). Modularity and community structure in networks. Proceedings of the National Academy of Sciences, 103(23):8577-8582.

North, D. C. (1991). Institutions. Journal of Economic Perspectives, 5(1):97-112.

Nowak, M. A. (2006). Five rules for the evolution of cooperation. Science, 314(5805):1560-1563.

Nowak, M. A. and May, R. M. (1992). Evolutionary games and spatial chaos. Nature, 359(6398):826-829.

Nowak, M. A. and May, R. M. (1993). The spatial dilemmas of evolution. International Journal of bifurcation and chaos, 3(01):35-78.

Papineau, D. (2002). Thinking about consciousness. Clarendon Press.

Perc, M., Gomez-Gardenes, J., Szolnoki, A., Floría, L. M., and Moreno, Y. (2013). Evolutionary dynamics of group interactions on structured populations: a review. Journal of The Royal Society Interface, 10(80):20120997. 
Perc, M., Jordan, J. J., Rand, D. G., Wang, Z., Boccaletti, S., and Szolnoki, A. (2017). Statistical physics of human cooperation. Physics Reports, 687:1-51.

Perc, M. and Szolnoki, A. (2008). Social diversity and promotion of cooperation in the spatial prisoner's dilemma game. Physical Review E, 77(1):011904.

Perc, M. and Szolnoki, A. (2010). Coevolutionary games - a mini review. BioSystems, 99(2):109-125.

Pons, P. and Latapy, M. (2006). Computing communities in large networks using random walks. J. Graph Algorithms Appl., 10(2):191-218.

Portes, A. and Vickstrom, E. (2011). Diversity, social capital, and cohesion. Annual Review of Sociology, 37:461-479.

Putnam, R. D. (2000). Bowling alone: America's declining social capital. In Culture and politics, pages 223-234. Springer.

Raub, W., Buskens, V., and Corten, R. (2015). Social dilemmas and cooperation. In Handbuch Modellbildung und Simulation in den Sozialwissenschaften, pages 597-626. Springer.

Raub, W., Buskens, V., and Van Assen, M. A. (2011). Micro-macro links and microfoundations in sociology. The Journal of Mathematical Sociology, 35(1$3): 1-25$.

Raub, W. and Weesie, J. (1990). Reputation and efficiency in social interactions: An example of network effects. American Journal of Sociology, 96(3):626-654.

Reyes, M. G. (2019). Frustrated equilibrium of asymmetric coordinating dynamics in a marketing game. In Proceedings of SAI Intelligent Systems Conference, pages 141-159. Springer.

Roca, C. P., Cuesta, J. A., and Sánchez, A. (2009). Effect of spatial structure on the evolution of cooperation. Physical Review E, 80(4):046106.

Roca, C. P., Lozano, S., Arenas, A., and Sánchez, A. (2010). Topological traps control flow on real networks: The case of coordination failures. PLoS One, $5(12)$.

Rothstein, B. and Stolle, D. (2008). The state and social capital: An institutional theory of generalized trust. Comparative Politics, 40(4):441-459.

Sakoda, J. M. (1971). The checkerboard model of social interaction. The Journal of Mathematical Sociology, 1(1):119-132. 
Santos, F. C., Pacheco, J. M., and Lenaerts, T. (2006). Evolutionary dynamics of social dilemmas in structured heterogeneous populations. Proceedings of the National Academy of Sciences of the United States of America, 103(9):34903494.

Schelling, T. C. (1969). Models of segregation. The American Economic Review, $59(2): 488-493$.

Schelling, T. C. (1971). Dynamic models of segregation. Journal of Mathematical Sociology, 1(2):143-186.

Schelling, T. C. (1980). The strategy of conflict. Harvard university press.

Sheleff, L. S. (1975). From restitutive law to repressive law durkheim's the division of labor in society re-visited. European Journal of Sociology/Archives Européennes de Sociologie, 16(1):16-45.

Smith, A. ([1937] 1776). The wealth of nations.

Smith, T. W., Davern, M., Freese, J., and Morgan, S. L. (1972-2018). General social surveys.

Spector, P. E. and Brannick, M. T. (2011). Methodological urban legends: The misuse of statistical control variables. Organizational Research Methods, $14(2): 287-305$.

Stauffer, D. (2008). Social applications of two-dimensional ising models. American Journal of Physics, 76(4):470-473.

Stegehuis, C., van der Hofstad, R., and van Leeuwaarden, J. S. (2016). Epidemic spreading on complex networks with community structures. Scientific Reports, 6 .

Stekhoven, D. J. and Bühlmann, P. (2011). Missforest-non-parametric missing value imputation for mixed-type data. Bioinformatics, 28(1):112-118.

Szabó, G. and Fath, G. (2007). Evolutionary games on graphs. Physics Reports, 446(4-6):97-216.

Szolnoki, A. and Perc, M. (2014). Coevolutionary success-driven multigames. EPL (Europhysics Letters), 108(2):28004.

Szolnoki, A., Perc, M., and Danku, Z. (2008). Towards effective payoffs in the prisoner's dilemma game on scale-free networks. Physica A: Statistical Mechanics and its Applications, 387(8-9):2075-2082. 
Tacchella, A., Cristelli, M., Caldarelli, G., Gabrielli, A., and Pietronero, L. (2012). A New Metrics for Countries' Fitness and Products' Complexity. Scientific Reports, 2:1-4.

Tan, S. J. and Tambyah, S. K. (2011). Generalized trust and trust in institutions in confucian asia. Social Indicators Research, 103(3):357-377.

Tomassini, M. and Pestelacci, E. (2010). Evolution of coordination in social networks: A numerical study. International Journal of Modern Physics $C$, 21(10):1277-1296.

van Gerwen, N. and Buskens, V. (2018). Partner-specific behavior in social networks: Coordination among actors with heterogeneous preferences. Social Networks, 52:68-80.

Van Lange, P. A., Joireman, J., Parks, C. D., and Van Dijk, E. (2013). The psychology of social dilemmas: A review. Organizational Behavior and Human Decision Processes, 120(2):125-141.

Voss, T. (2001). Game-theoretical perspectives on the emergence of social norms. na.

Vriens, E. and Corten, R. (2018). Are bridging ties really advantageous? an experimental test of their advantage in a competitive social learning context. Social Networks, 54:91-100.

Waldrop, M. M. (1993). Complexity: The emerging science at the edge of order and chaos. Simon and Schuster.

Walentek, D., Broere, J., Cinelli, M., Dekker, M., and Haslbeck, J. (2020). Success of economic sanctions threats: Coercion, information and commitment. International Interactions.

Wallace, G. P. (2013). Regime type, issues of contention, and economic sanctions: Re-evaluating the economic peace between democracies. Journal of Peace Research, 50(4):479-493.

Wang, Z., Szolnoki, A., and Perc, M. (2014). Different perceptions of social dilemmas: Evolutionary multigames in structured populations. Physical Review E, 90(3):032813.

Watts, D. J. (2011). Everything is obvious:* Once you know the answer. Crown Business. 
Watts, D. J. and Dodds, P. S. (2007). Influentials, networks, and public opinion formation. Journal of Consumer Research, 34(4):441-458.

Watts, D. J. and Strogatz, S. H. (1998). Collective dynamics of 'smallworld'networks. Nature, 393(6684):440-442.

Wikipedia (2019). Wikipedia. https://en.wikipedia.org/wiki/Sociology. Accessed: 2019-12-31.

xkdc (2019). Fields aranged by purity. https://imgs.xkcd.com/comics/purity.png. Accessed: 2019-11-23. 

Dankwoord (Acknowledgements) 



\section{Dankwoord (Acknowledgements)}

Er wordt vaak gezegd dat het schrijven van een dissertatie een "eenzaam gevecht" is $^{2}{ }^{3}$, maar zo heb ik het dankzij een fanstasische groep mensen om mij heen helemaal niet ervaren. Het is een proces geweest met tal van mooie ontmoetingen, inspirerende samenwerkingen en plezier, tijdens en naast het werk, met mensen waarmee ik mij gelukkig prijs dat ik ze mijn collega's en vrienden kan noemen. Zonder hun was het zeker niet gelukt deze dissertatie schrijven en daarom ben ik een uitgebreid dankwoord schuldig.

Te beginnen bij mijn promotoren Vincent en Henk. Ik heb erg veel geluk gehad met twee promotoren van dit kaliber. Niet alleen zijn jullie beide intimiderend goed in je werk, ook zijn jullie beide enorm betrokken bij je studenten, iets wat niet voor lief te nemen is. Vincent, toen ik aan het einde van mij master opzoek was naar een $\mathrm{PhD}$-positie is mij meermaals gevraagd of ik dat in de richting methodologie en statistiek of ook sociologie wilde doen. Ik antwoordde steevast dat ik alleen een $\mathrm{PhD}$ in de richting sociologie wilde doen als dit kon bij Vincent Buskens. Ik twijfelde geen moment toen je even later vroeg of ik op een positie wilde solliciteren. Je kantoor deur stond (letterlijk en figuurlijk) altijd open. Als ik onverwacht binnen kwam lopen wist je altijd gelijk waar ik het over had en wist me gelijk verder te helpen. Het moment dat we samen bier aan het tappen waren achter de bar bij IMEBSS zal ik niet meer vergeten. Henk, tijdens onze meetings heb ik me regelmatig gevoeld zoals het karakter dat Bill Murray speelt in Lost in Translation zich gevoeld moet hebben. Desalniettemin had ik vaak een tegenovergesteld gevoel vlak na onze meetings als je toch weer precies de opmerking had gemaakt die me verder hielp. Bedankt voor soms je geduld, maar altijd je goede opmerkingen.

Dan wil ik graag Peter Koeze bedanken zonder wie deze PhD niet mogelijk was geweest. Peter heeft zelf succes gehad door zijn kennis uit de natuurkunde toe te passen op andere terreinen. De opdracht was dan ook om natuurkundige technieken te combineren met andere wetenschappelijke gebieden. Dit gaf ruimte en vrijheid voor onderzoek van meer fundamentele aard. Ook tijdens de PhD was Peter altijd zeer betrokken, altijd op de hoogte en wist altijd de juiste vragen te stellen. Dankzij Peter was dit vanaf het begin af aan een bijzonder project.

\footnotetext{
${ }^{2}$ https://www.trouw.nl/nieuws/promovendus-kom-eens-achter-dat-bureauvandaan b50e80e4

${ }^{3}$ https://www.advalvas.vu.nl/tip/promoveren-hoeft-niet-alleen-11-tips
} 
Zonder mijn kamergenoten waren de afgelopen 4 jaar niet hetzelfde geweest. Jorans, bedankt dat iedere dag op het kantoor als een feestje aanvoelde, door jullie ging ik iedere dag met plezier naar mijn werk. Jannes, bedankt voor een 3 jaar durende indoor kantoor voetbal competentie eindigend in 163-163 (sorry Wojtek), de dansjes, het eindeloze geouwehoer en alle avonturen tijdens en na het werk. Marcus bedankt voor een mooi artikel samen, dat je me hebt leren langlaufen en twee keer Scandinavië hebt laten zien. Dat zal ik niet meer vergeten. Sanne, bedankt dat je altijd ofwel lekker meedeed met alle onzin ofwel de onzin tolereerde. Ik hoop je snel te zien als nieuwe collega bij het WODC.

The neighbors: Eva, Judith, Mathijs en Siyang, and the neighbors a few doors down: Nikki, Leonie, Müge and Lex. That we had multiple "best weekends ever" could logically only happen because they kept getting better. Thanks for all the lunches, walks through the botanical gardens, swimming at Amelisweerd (although the water there is a bit gross), discussions about all the things that were not sociology, the parties and the trips. But mostly thanks that it did not feels once like we were colleagues, and with you around it never felt like work.

Thanks to all participants of the cooperative relations seminar: Rense, Joyce, Wojtek, Arnout, Kasper, Werner, Rita, Jeroen, Phillipp, Hendrik, Lucas, Charlotte, those who I have mentioned above already, and those who joined infrequently, thanks for your valuable discussions.

I would like to thank the reading committee: Henk Dijkstra, Andreas Flache, Peter van der Heijden, Penélope Hernández and Arnout van de Rijt for their time, effort and comments on this dissertation.

Thanks to the people at Universidad Carlos III de Madrid for a wonderful stay in Madrid. Anxo, thank you for all the time and devotion you have put in the project. It felt like I had third supervisor. Pau, Pablo, Vikki and Ignacio, thank you for making me feel welcome, all the nice lunches during work and beers after work. Pau and Ignacio, a special thanks for your help with the experiments.

Thanks to everyone at the Centre for Complex Systems Studies, with a special thanks to Qingyi. Thanks to Alje, Dawid, Mark and Matteo for successful collaborations. Brian and Mara, I hope by the time you read this the simulations are done. Thanks for a nice collaboration.

Thanks to all the people at the Santa Fe summer school, which was an experience I'll never forget. A special thank you to Juste, Marius, Jesus Mario, Evelyn, 
Christine, Ben and Lorraine, for cooperation, the publication and the meetings in Paris and Amsterdam. I will make sure that the London meeting will still happen.

The Amsterdam group: Jonas, once we had a conversation about if we ever met someone in real life who you truly admired because they did something you could never do. I said I only met two such persons. At the time I did not mention the third, so let me say it now. Your dedication towards everything and everyone in your life is extraordinary, something I truly look up to. Thanks for all the adventures, thanks for being my friend. Giacomo, the dissertation cover will always remind me of you when I look back on this time in my life. Thanks for being part of it, for all the times you cooked us dinner and all amazing and crazy stories we have together. Johnny, Javier, Damiano, Fabian, Daniel, thanks for all the hikes, book clubs, amazing parties, adventures in nature, summers in the city and breakfast clubs. You are all amazing people.

Methods and stats group: Oisín, whenever I could not handle the second floor anymore I always went down to visit the first. Thanks that you were always there, for all the sense and nonsense conversations and for all the times I made you listen to short incoherent techno samples, luckily Prince made a two hour movie. Thomas, Roline, Millitza, Fayette, thanks for still meeting up after the masters, thanks making stats cool.

Vrienden uit Amersfoort: Rutger, Rick, Suus, Maarten, Anne, Joey en Marina. We kennen elkaar nu al bijna 20 jaar. Ondanks dat we elkaar nu minder zien is het elke keer alsof we elkaar gister nog gesproken hebben. Bedankt voor jullie onvoorwaardelijke vriendschap.

Iedereen bij CE promovendi groep van Stichting Maatschappij en Onderneming bedankt dat jullie me kennis hebben laten maken circulaire economie en ondernemen. Daphne en Bram in het bijzonder.

Mijn familie bedankt, voor mijn ouders, ik ben nu eindelijk 'afgestudeerd'. Lilian, Ruud, Noah en Amelie bedankt voor jullie steun op de momenten dat het nodig was.

Merel, je bent zonder twijfel de meest belangrijke persoon in mijn leven geweest de afgelopen vier jaar. Bedankt dat ik tijdens hoogte en diepte punten altijd op je kon rekenen, voor alle mooie momenten samen, op vakanties, op feestjes of gewoon thuis op bank. Zonder jou had ik het niet kunnen doen. 

Publications and working papers by the author 



\section{Publications and working papers by the author}

\section{Peer-Reviewed Publications}

Raimbault, J., Broere, J., Somveille, M., Serna, J.M., Strombom, E., Moore, C., \& Zhu, B., Sugar, L. A. (2020). A spatial agent based model for simulating and optimizing networked eco-industrial systems Resources, Conservation and Recycling.

Walentek, D., Broere, J., Cinelli, M., Dekker, M., \& Haslbeck, J. (2020). Success of Economic Sanctions Threats: Coercion, Information and Commitment International Interactions.

Broere, J.J., Buskens, V., Stoof, H.T.C., \& Sanchez, A. (2019). An experimental study of network effects on coordination in asymmetric games. Scientific Reports.

Broere, J.J., Buskens, V., Weesie, J., \& Stoof, H.T.C. (2017). Network effects on coordination in asymmetric games. Scientific Reports.

Van de Schoot, R., Broere, J.J., Perryck, K., Zondervan-Zwijnenburg, M., \& Van Loey, N. (2015). Analyzing Small Data Sets using Bayesian Estimation: The case of posttraumatic stress symptoms following mechanical ventilation in burn survivors. European Journal of Psychotraumatology.

\section{Other Publications}

Bet, B., Kas, J., D. Truijens, Lee, S. van der, Broere, J., Leising, E., Nuninga, T., Bose, P., Ravensberg, E. van, Francesco, E. di, Wang, Y, Hassan, A., Fanitabasi, F., Wang, Z. (2018). Barriers and Best Practices for the Circular Economy: SMO Promovendi-Circular Minds. Stichting Maatschappij en Onderneming (SMO).

Bet, B., de Ritter,M., Broere, J., D. Truijens, Lee, S. van der, Hees, S., Vernooij, J., Schreuder Hes, M., Roelvink, S. (2017). De sociale en economische complexiteit van Industriele symbiose. Book chapter in: Circulaire Economie: Wat? Waarom? Hoe? Stichting Maatschappij en Onderneming (SMO).

\section{Under Review and Work in Progress}

Broere, J.J., van Dam, A. \& Kristiansen M.H. The division of labour and generalized trust: Organic solidarity revisited.

Broere, J.J., Buskens, V., \& Stoof, H.T.C. Asymmetric games on networks and the Ising model.

Dekker, M., Walentek, D., Haslbeck, J., \& Broere, J. A General Framework for International Conflict under Incomplete Information. 
Dermody, B., Broere, J. \& Baudena, M. Emergence of cooperation in sociohydrological landscapes. 

ICS Dissertation Series 


\section{ICS Dissertation Series}

The ICS-series presents dissertations of the Interuniversity Center for Social Science Theory and Methodology. Each of these studies aims at integrating explicit theory formation with state-of-the-art empirical research or at the development of advanced methods for empirical research. The ICS was founded in 1986 as a cooperative effort of the universities of Groningen and Utrecht. Since 1992, the ICS expanded to the University of Nijmegen and since 2017 to the University of Amsterdam (UvA). Most of the projects are financed by the participating universities or by the Netherlands Organization for Scientific Research (NWO). The international composition of the ICS graduate students is mirrored in the increasing international orientation of the projects and thus of the ICS-series itself.

1. C. van Liere. (1990). Lastige leerlingen. Een empirisch onderzoek naar sociale oorzaken van probleemgedrag op basisscholen. Amsterdam: Thesis Publishers.

2. Marco H.D. van Leeuwen. (1990). Bijstand in Amsterdam, ca. 1800-1850. Armenzorg als beheersings- en overlevingsstrategie. ICS-dissertation, Utrecht.

3. I. Maas. (1990). Deelname aan podiumkunsten via de podia, de media en actieve beoefening. Substitutie of leereffecten?. Amsterdam: Thesis Publishers.

4. M.I. Broese van Groenou. (1991). Gescheiden netwerken. De relaties met vrienden en verwanten na echtscheiding. Amsterdam: Thesis Publishers.

5. Jan M.M. van den Bos. (1991). Dutch EC policy making. A model-guided approach to coordination and negotiation. Amsterdam: Thesis Publishers.

6. Karin Sanders. (1991). Vrouwelijke pioniers. Vrouwen en mannen met een 'mannelijke' hogere beroepsopleiding aan het begin van hun loopbaan. Amsterdam: Thesis Publishers.

7. Sjerp de Vries. (1991). Egoism, altruism, and social justice. Theory and experiments on cooperation in social dilemmas. Amsterdam: Thesis Publishers.

8. Ronald S. Batenburg. (1991). Automatisering in bedriff. Amsterdam: Thesis Publishers.

9. Rudi Wielers. (1991). Selectie en allocatie op de arbeidsmarkt. Een uitwerking voor de informele en geïstitutionaliseerde kinderopvang. Amsterdam: Thesis Publishers.

10. Gert P. Westert. (1991). Verschillen in ziekenhuisgebruik. ICS-dissertation, Groningen.

11. Hanneke Hermsen. (1992). Votes and policy preferences. Equilibria in party systems. Amsterdam: Thesis Publishers.

12. Cora J.M. Maas. (1992). Probleemleerlingen in het basisonderwijs. Amsterdam: Thesis Publishers. 
13. Ed A.W. Boxman. (1992). Contacten en carrière. Een empirisch-theoretisch onderzoek naar de relatie tussen sociale netwerken en arbeidsmarktposities. Amsterdam: Thesis Publishers.

14. Conny G.J. Taes. (1992). Kijken naar banen. Een onderzoek naar de inschatting van arbeidsmarktkansen bij schoolverlaters uit het middelbaar beroepsonderwijs. Amsterdam: Thesis Publishers.

15. Peter van Roozendaal. (1992). Cabinets in multi-party democracies. The effect of dominant and central parties on cabinet composition and durability. Amsterdam: Thesis Publishers.

16. Marcel van Dam. (1992). Regio zonder regie. Verschillen in en effectiviteit van gemeentelijk arbeidsmarktbeleid. Amsterdam: Thesis Publishers.

17. Tanja van der Lippe. (1993). Arbeidsverdeling tussen mannen en vrouwen. Amsterdam: Thesis Publishers.

18. Marc A. Jacobs. (1993). Software: Kopen of kopiëren? Een sociaal-wetenschappelijk onderzoek onder PC-gebruikers. Amsterdam: Thesis Publishers.

19. Peter van der Meer. (1993). Verdringing op de Nederlandse arbeidsmarkt. Sectoren sekseverschillen. Amsterdam: Thesis Publishers.

20. Gerbert Kraaykamp. (1993). Over lezen gesproken. Een studie naar sociale differentiatie in leesgedrag. Amsterdam: Thesis Publishers.

21. Evelien Zeggelink. (1993). Strangers into friends. The evolution of friendship networks using an individual oriented modeling approach. Amsterdam: Thesis Publishers.

22. Jaco Berveling. (1994). Het stempel op de besluitvorming. Macht, invloed en besluitvorming op twee Amsterdamse beleidsterreinen. Amsterdam: Thesis Publishers.

23. Wim Bernasco. (1994). Coupled careers. The effects of spouse's resources on success at work. Amsterdam: Thesis Publishers.

24. Liset van Dijk. (1994). Choices in child care. The distribution of child care among mothers, fathers and non-parental care providers. Amsterdam: Thesis Publishers.

25. Jos de Haan. (1994). Research groups in Dutch sociology. Amsterdam: Thesis Publishers.

26. K. Boahene. (1995). Innovation adoption as a socio-economic process. The case of the Ghanaian cocoa industry. Amsterdam: Thesis Publishers.

27. Paul E.M. Ligthart. (1995). Solidarity in economic transactions. An experimental study of framing effects in bargaining and contracting. Amsterdam: Thesis Publishers.

28. Roger Th. A.J. Leenders. (1995). Structure and influence. Statistical models for the dynamics of actor attributes, network structure, and their interdependence. Amsterdam: Thesis Publishers.

29. Beate Völker. (1995). Should auld acquaintance be forgot...? Institutions of communism, the transition to capitalism and personal networks: The case of East Germany. Amsterdam: Thesis Publishers. 
30. A. Cancrinus-Matthijsse. (1995). Tussen hulpverlening en ondernemerschap. Beroepsuitoefening en taakopvattingen van openbare apothekers in een aantal WestEuropese landen. Amsterdam: Thesis Publishers.

31. Nardi Steverink. (1996). Zo lang mogelijk zelfstandig. Naar een verklaring van verschillen in oriëntatie ten aanzien van opname in een verzorgingstehuis onder fysiek kwetsbare ouderen. Amsterdam: Thesis Publishers.

32. Ellen Lindeman. (1996). Participatie in vrijwilligerswerk. Amsterdam: Thesis Publishers.

33. Chris Snijders. (1996). Trust and commitments. Amsterdam: Thesis Publishers.

34. Koos Postma. (1996). Changing prejudice in Hungary. A study on the collapse of state socialism and its impact on prejudice against gypsies and Jews. Amsterdam: Thesis Publishers.

35. Jooske T. van Busschbach. (1996). Uit het oog, uit het hart? Stabiliteit en verandering in persoonlijke relaties. Amsterdam: Thesis Publishers.

36. René Torenvlied. (1996). Besluiten in uitvoering. Theorieën over beleidsuitvoering modelmatig getoetst op sociale vernieuwing in drie gemeenten. Amsterdam: Thesis Publishers.

37. Andreas Flache. (1996). The Double edge of networks. An analysis of the effect of informal networks on cooperation in social dilemmas. Amsterdam: Thesis Publishers.

38. Kees van Veen. (1997). Inside an internal labor market: Formal rules, flexibility and career lines in a Dutch manufacturing company. Amsterdam: Thesis Publishers.

39. Lucienne van Eijk. (1997). Activity and well-being in the elderly. Amsterdam: Thesis Publishers.

40. Róbert Gál. (1997). Unreliability. Contract discipline and contract governance under economic transition. Amsterdam: Thesis Publishers.

41. Anne-Geerte van de Goor. (1997). Effects of regulation on disability duration. ICS-dissertation, Utrecht.

42. Boris Blumberg. (1997). Das Management von Technologiekooperationen. Partnersuche und Verhandlungen mit dem Partner aus Empirisch-Theoretischer Perspektive. ICS-dissertation, Utrecht.

43. Marijke von Bergh. (1997). Loopbanen van oudere werknemers. Amsterdam: Thesis Publishers.

44. Anna Petra Nieboer. (1997). Life-events and well-being: A prospective study on changes in well-being of elderly people due to a serious illness event or death of the spouse. Amsterdam: Thesis Publishers.

45. Jacques Niehof. (1997). Resources and social reproduction: The effects of cultural and material resources on educational and occupational careers in industrial nations at the end of the twentieth century. ICS-dissertation, Nijmegen. 
46. Ariana Need. (1997). The kindred vote. Individual and family effects of social class and religion on electoral change in the Netherlands, 1956-1994. ICS-dissertation, Nijmegen.

47. Jim Allen. (1997). Sector composition and the effect of education on wages: An international comparison. Amsterdam: Thesis Publishers.

48. Jack B.F. Hutten. (1998). Workload and provision of care in general practice. An empirical study of the relation between workload of Dutch general practitioners and the content and quality of their care. ICS-dissertation, Utrecht.

49. Per B. Kropp. (1998). Berufserfolg im Transformationsprozeß, Eine theoretischempirische Studie über die Gewinner und Verlierer der Wende in Ostdeutschland. ICS-dissertation, Utrecht.

50. Maarten H.J. Wolbers. (1998). Diploma-inflatie en verdringing op de arbeidsmarkt. Een studie naar ontwikkelingen in de opbrengsten van diploma's in Nederland. ICS-dissertation, Nijmegen.

51. Wilma Smeenk. (1998). Opportunity and marriage. The impact of individual resources and marriage market structure on first marriage timing and partner choice in the Netherlands. ICS-dissertation, Nijmegen.

52. Marinus Spreen. (1999). Sampling personal network structures: Statistical inference in ego-graphs. ICS-dissertation, Groningen.

53. Vincent Buskens. (1999). Social networks and trust. ICS-dissertation, Utrecht.

54. Susanne Rijken. (1999). Educational expansion and status attainment. A crossnational and over-time comparison. ICS-dissertation, Utrecht.

55. Mérove Gijsberts. (1999). The legitimation of inequality in state-socialist and market societies, 1987-1996. ICS-dissertation, Utrecht.

56. Gerhard G. Van de Bunt. (1999). Friends by choice. An actor-oriented statistical network model for friendship networks through time. ICS-dissertation, Groningen.

57. Robert Thomson. (1999). The party mandate: Election pledges and government actions in the Netherlands, 1986-1998. Amsterdam: Thela Thesis.

58. Corine Baarda. (1999). Politieke besluiten en boeren beslissingen. Het draagvlak van het mestbeleid tot 2000. ICS-dissertation, Groningen.

59. Rafael Wittek. (1999). Interdependence and informal control in organizations. ICS-dissertation, Groningen.

60. Diane Payne. (1999). Policy making in the European Union: An analysis of the impact of the reform of the structural funds in Ireland. ICS-dissertation, Groningen.

61. René Veenstra. (1999). Leerlingen - klassen - scholen. Prestaties en vorderingen van leerlingen in het voortgezet onderwijs. Amsterdam: Thela Thesis.

62. Marjolein Achterkamp. (1999). Influence strategies in collective decision making. A comparison of two models. ICS-dissertation, Groningen.

63. Peter Mühlau. (2000). The governance of the employment relation. A relational signaling perspective. ICS-dissertation, Groningen. 
64. Agnes Akkerman. (2000). Verdeelde vakbeweging en stakingen. Concurrentie om leden. ICS-dissertation, Groningen.

65. Sandra van Thiel. (2000). Quangocratization: Trends, causes and consequences. ICS-dissertation, Utrecht.

66. Rudi Turksema. (2000). Supply of day care. ICS-dissertation, Utrecht.

67. Sylvia E. Korupp (2000). Mothers and the process of social stratification. ICSdissertation, Utrecht.

68. Bernard A. Nijstad (2000). How the group affects the mind: Effects of communication in idea generating groups. ICS-dissertation, Utrecht.

69. Inge F. de Wolf (2000). Opleidingsspecialisatie en arbeidsmarktsucces van sociale wetenschappers. ICS-dissertation, Utrecht.

70. Jan Kratzer (2001). Communication and performance: An empirical study in innovation teams. ICS-dissertation, Groningen.

71. Madelon Kroneman (2001). Healthcare systems and hospital bed use. ICS/NIVELdissertation, Utrecht.

72. Herman van de Werfhorst (2001). Field of study and social inequality. Four types of educational resources in the process of stratification in the Netherlands. ICSdissertation, Nijmegen.

73. Tamás Bartus (2001). Social capital and earnings inequalities. The role of informal job search in Hungary. ICS-dissertation, Groningen.

74. Hester Moerbeek (2001). Friends and foes in the occupational career. The influence of sweet and sour social capital on the labour market. ICS-dissertation, Nijmegen.

75. Marcel van Assen (2001). Essays on actor perspectives in exchange networks and social dilemmas. ICS-dissertation, Groningen.

76. Inge Sieben (2001). Sibling similarities and social stratification. The impact of family background across countries and cohorts. ICS-dissertation, Nijmegen.

77. Alinda van Bruggen (2001). Individual production of social well-being. An exploratory study. ICS-dissertation, Groningen.

78. Marcel Coenders (2001). Nationalistic attitudes and ethnic exclusionism in a comparative perspective: An empirical study of attitudes toward the country and ethnic immigrants in 22 countries. ICS-dissertation, Nijmegen.

79. Marcel Lubbers (2001). Exclusionistic electorates. Extreme right-wing voting in Western Europe. ICS-dissertation, Nijmegen.

80. Uwe Matzat (2001). Social networks and cooperation in electronic communities. A theoretical-empirical analysis of academic communication and internet discussion groups. ICS-dissertation, Groningen.

81. Jacques P.G. Janssen (2002). Do opposites attract divorce? Dimensions of mixed marriage and the risk of divorce in the Netherlands. ICS-dissertation, Nijmegen.

82. Miranda Jansen (2002). Waardenoriëntaties en partnerrelaties. Een panelstudie naar wederzijdse invloeden. ICS-dissertation, Utrecht. 
83. Anne Rigt Poortman (2002). Socioeconomic causes and consequences of divorce. ICS-dissertation, Utrecht.

84. Alexander Gattig (2002). Intertemporal decision making. ICS-dissertation, Groningen.

85. Gerrit Rooks (2002). Contract en conflict: Strategisch management van inkooptransacties. ICS-dissertation, Utrecht.

86. Károly Takács (2002). Social networks and intergroup conflict. ICS-dissertation, Groningen.

87. Thomas Gautschi (2002). Trust and exchange, effects of temporal embeddedness and network embeddedness on providing and dividing a surplus. ICS-dissertation, Utrecht.

88. Hilde Bras (2002). Zeeuwse meiden. Dienen in de levensloop van vrouwen, ca. 1850-1950. Amsterdam: Aksant Academic Publishers.

89. Merijn Rengers (2002). Economic lives of artists. Studies into careers and the labour market in the cultural sector. ICS-dissertation, Utrecht.

90. Annelies Kassenberg (2002). Wat scholieren bindt. Sociale gemeenschap in scholen. ICS-dissertation, Groningen

91. Marc Verboord (2003). Moet de meester dalen of de leerling klimmen? De invloed van literatuuronderwijs en ouders op het lezen van boeken tussen 1975 en 2000. ICS-dissertation, Utrecht.

92. Marcel van Egmond (2003). Rain falls on all of us (but some manage to get more wet than others): Political Context and Electoral Participation. ICS-dissertation, Nijmegen.

93. Justine Horgan (2003). High performance human resource management in Ireland and the Netherlands: Adoption and effectiveness. ICS-dissertation, Groningen.

94. Corine Hoeben (2003). LETS' be a community. Community in local exchange trading systems. ICS-dissertation, Groningen.

95. Christian Steglich (2003). The framing of decision situations. Automatic goal selection and rational goal pursuit. ICS-dissertation, Groningen.

96. Johan van Wilsem (2003). Crime and context. The impact of individual, neighborhood, city and country characteristics on victimization. ICS-dissertation, Nijmegen.

97. Christiaan Monden (2003). Education, inequality and health. The impact of partners and life course. ICS-dissertation, Nijmegen.

98. Evelyn Hello (2003). Educational attainment and ethnic attitudes. How to explain their relationship. ICS-dissertation, Nijmegen.

99. Marnix Croes en Peter Tammes (2004). Gif laten wij niet voortbestaan. Een onderzoek naar de overlevingskansen van joden in de Nederlandse gemeenten, 19401945. Amsterdam: Aksant Academic Publishers.

100. Ineke Nagel (2004). Cultuurdeelname in de levensloop. ICS-dissertation, Utrecht. 
101. Marieke van der Wal (2004). Competencies to participate in life. Measurement and the impact of school. ICS-dissertation, Groningen.

102. Vivian Meertens (2004). Depressive symptoms in the general population: A multifactorial social approach. ICS-dissertation, Nijmegen.

103. Hanneke Schuurmans (2004). Promoting well-being in frail elderly people. Theory and intervention. ICS-dissertation, Groningen.

104. Javier Arregui (2004). Negotiation in legislative decision-making in the European Union. ICS-dissertation, Groningen.

105. Tamar Fischer (2004). Parental divorce, conflict and resources. The effects on children's behaviour problems, socioeconomic attainment, and transitions in the demographic career. ICS-dissertation, Nijmegen.

106. René Bekkers (2004). Giving and volunteering in the Netherlands: Sociological and psychological perspectives. ICS-dissertation, Utrecht.

107. Renée van der Hulst (2004). Gender differences in workplace authority: An empirical study on social networks. ICS-dissertation, Groningen.

108. Rita Smaniotto (2004). 'You scratch my back and I scratch yours' versus 'Love thy neighbour'. Two Proximate Mechanisms of Reciprocal Altruism. ICS-dissertation, Groningen.

109. Maurice Gesthuizen (2004). The life-course of the low-educated in the Netherlands: Social and economic risks. ICS-dissertation, Nijmegen.

110. Carlijne Philips (2005). Vakantiegemeenschappen. Kwalitatief en kwantitatief onderzoek naar gelegenheid- en refreshergemeenschap tijdens de vakantie. ICSdissertation, Groningen.

111. Esther de Ruijter (2005). Household outsourcing. ICS-dissertation, Utrecht.

112. Frank van Tubergen (2005). The integration of immigrants in cross-national perspective: Origin, destination, and community effects. ICS-dissertation, Utrecht.

113. Ferry Koster (2005). For the time being. Accounting for inconclusive findings concerning the effects of temporary employment relationships on solidary behavior of employees. ICS-dissertation, Groningen.

114. Carolien Klein Haarhuis (2005). Promoting anti-corruption reforms. Evaluating the implementation of a World Bank anti-corruption program in seven African countries (1999-2001). ICS-dissertation, Utrecht.

115. Martin van der Gaag (2005). Measurement of individual social capital. ICS-dissertation, Groningen.

116. Johan Hansen (2005). Shaping careers of men and women in organizational contexts. ICS-dissertation, Utrecht.

117. Davide Barrera (2005). Trust in embedded settings. ICS-dissertation, Utrecht.

118. Mattijs Lambooij (2005). Promoting cooperation. Studies into the effects of long-term and short-term rewards on cooperation of employees. ICS-dissertation, Utrecht. 
119. Lotte Vermeij (2006). What's cooking? Cultural boundaries among Dutch teenagers of different ethnic origins in the context of school. ICS-dissertation, Utrecht.

120. Mathilde Strating (2006). Facing the challenge of rheumatoid arthritis. A 13-year prospective study among patients and cross-sectional study among their partners. ICS-dissertation, Groningen.

121. Jannes de Vries (2006). Measurement error in family background variables: The bias in the intergenerational transmission of status, cultural consumption, party preference, and religiosity. ICS-dissertation, Nijmegen.

122. Stefan Thau (2006). Workplace deviance: Four studies on employee motives and self-regulation. ICS-dissertation, Groningen.

123. Mirjam Plantinga (2006). Employee motivation and employee performance in child care. The effects of the introduction of market forces on employees in the Dutch child-care sector. ICS-dissertation, Groningen.

124. Helga de Valk (2006). Pathways into adulthood. A comparative study on family life transitions among migrant and Dutch youth. ICS-dissertation, Utrecht.

125. Henrike Elzen (2006). Self-management for chronically ill older people. ICS-dissertation, Groningen.

126. Ayşe Güveli (2007). New social classes within the service class in the Netherlands and Britain. Adjusting the EGP class schema for the technocrats and the social and cultural specialists. ICS-dissertation, Nijmegen.

127. Willem-Jan Verhoeven (2007). Income attainment in post-communist societies. ICS-dissertation, Utrecht.

128. Marieke Voorpostel (2007). Sibling support: The exchange of help among brothers and sisters in the Netherlands. ICS-dissertation, Utrecht.

129. Jacob Dijkstra (2007). The effects of externalities on partner choice and payoffs in exchange networks. ICS-dissertation, Groningen.

130. Patricia van Echtelt (2007). Time-greedy employment relationships: Four studies on the time claims of post-Fordist work. ICS-dissertation, Groningen.

131. Sonja Vogt (2007). Heterogeneity in social dilemmas: The case of social support. ICS-dissertation, Utrecht.

132. Michael Schweinberger (2007). Statistical methods for studying the evolution of networks and behavior. ICS-dissertation, Groningen.

133. István Back (2007). Commitment and evolution: Connecting emotion and reason in long-term relationships. ICS-dissertation, Groningen.

134. Ruben van Gaalen (2007). Solidarity and ambivalence in parent-child relationships. ICS-dissertation, Utrecht.

135. Jan Reitsma (2007). Religiosity and solidarity - Dimensions and relationships disentangled and tested. ICS-dissertation, Nijmegen.

136. Jan Kornelis Dijkstra (2007) Status and affection among (pre)adolescents and their relation with antisocial and prosocial behavior. ICS-dissertation, Groningen. 
137. Wouter van Gils (2007). Full-time working couples in the Netherlands. Causes and consequences. ICS-dissertation, Nijmegen.

138. Djamila Schans (2007). Ethnic diversity in intergenerational solidarity. ICSdissertation, Utrecht.

139. Ruud van der Meulen (2007). Brug over woelig water: Lidmaatschap van sportverenigingen, vriendschappen, kennissenkringen en veralgemeend vertrouwen. ICSdissertation, Nijmegen.

140. Andrea Knecht (2008). Friendship selection and friends' influence. Dynamics of networks and actor attributes in early adolescence. ICS-dissertation, Utrecht.

141. Ingrid Doorten (2008). The division of unpaid work in the household: A stubborn pattern?. ICS-dissertation, Utrecht.

142. Stijn Ruiter (2008). Association in context and association as context: Causes and consequences of voluntary association involvement. ICS-dissertation, Nijmegen.

143. Janneke Joly (2008). People on our minds: When humanized contexts activate social norms. ICS-dissertation, Groningen.

144. Margreet Frieling (2008). 'Joint production' als motor voor actief burgerschap in de buurt. ICS-dissertion, Groningen.

145. Ellen Verbakel (2008). The partner as resource or restriction? Labour market careers of husbands and wives and the consequences for inequality between couples. ICS-dissertation, Nijmegen.

146. Gijs van Houten (2008). Beleidsuitvoering in gelaagde stelsels. De doorwerking van aanbevelingen van de Stichting van de Arbeid in het CAO-overleg. ICSdissertation, Utrecht.

147. Eva Jaspers (2008). Intolerance over time. Macro and micro level questions on attitudes towards euthanasia, homosexuality and ethnic minorities. ICS-dissertation, Nijmegen.

148. Gijs Weijters (2008). Youth delinquency in Dutch cities and schools: A multilevel approach. ICS-dissertation, Nijmegen.

149. Jessica Pass (2009). The self in social rejection. ICS-dissertation, Groningen.

150. Gerald Mollenhorst (2009). Networks in contexts. How meeting opportunities affect personal relationships. ICS-dissertation, Utrecht.

151. Tom van der Meer (2009). States of freely associating citizens: Comparative studies into the impact of state institutions on social, civic and political participation. ICS-dissertation, Nijmegen.

152. Manuela Vieth (2009). Commitments and reciprocity in trust situations. Experimental studies on obligation, indignation, and self-consistency. ICS-dissertation, Utrecht.

153. Rense Corten (2009). Co-evolution of social networks and behavior in social dilemmas: Theoretical and empirical perspectives. ICS-dissertation, Utrecht.

154. Arieke J. Rijken (2009). Happy families, high fertility? Childbearing choices in the context of family and partner relationships. ICS-dissertation, Utrecht. 
155. Jochem Tolsma (2009). Ethnic hostility among ethnic majority and minority groups in the Netherlands. An investigation into the impact of social mobility experiences, the local living environment and educational attainment on ethnic hostility. ICS-dissertation, Nijmegen.

156. Freek Bucx (2009). Linked lives: Young adults' life course and relations with parents. ICS-dissertation, Utrecht.

157. Philip Wotschack (2009). Household governance and time allocation. Four studies on the combination of work and care. ICS-dissertation, Groningen.

158. Nienke Moor (2009). Explaining worldwide religious diversity. The relationship between subsistence technologies and ideas about the unknown in pre-industrial and (post-)industrial societies. ICS-dissertation, Nijmegen.

159. Lieke ten Brummelhuis (2009). Family matters at work. Depleting and enriching effects of employees' family lives on work outcomes. ICS-dissertation, Utrecht.

160. Renske Keizer (2010). Remaining childless. Causes and consequences from a life course perspective. ICS-dissertation, Utrecht.

161. Miranda Sentse (2010). Bridging contexts: The interplay between family, child, and peers in explaining problem behavior in early adolescence. ICS-dissertation, Groningen.

162. Nicole Tieben (2010). Transitions, tracks and transformations. Social inequality in transitions into, through and out of secondary education in the Netherlands for cohorts born between 1914 and 1985. ICS-dissertation, Nijmegen.

163. Birgit Pauksztat (2010). Speaking up in organizations: Four studies on employee voice. ICS-dissertation, Groningen.

164. Richard Zijdeman (2010). Status attainment in the Netherlands, 1811-1941. Spatial and temporal variation before and during industrialization. ICS-dissertation, Utrecht.

165. Rianne Kloosterman (2010). Social background and children's educational careers. The primary and secondary effects of social background over transitions and over time in the Netherlands. ICS-dissertation, Nijmegen.

166. Olav Aarts (2010). Religious diversity and religious involvement. A study of religious markets in Western societies at the end of the twentieth century. ICSdissertation, Nijmegen.

167. Stephanie Wiesmann (2010). 24/7 negotiation in couples transition to parenthood. ICS-dissertation, Utrecht.

168. Borja Martinovic (2010). Interethnic contacts: A dynamic analysis of interaction between immigrants and natives in Western countries. ICS-dissertation, Utrecht.

169. Anne Roeters (2010). Family life under pressure? Parents' paid work and the quantity and quality of parent-child and family time. ICS-dissertation, Utrecht.

170. Jelle Sijtsema (2010). Adolescent aggressive behavior: Status and stimulation goals in relation to the peer context. ICS-dissertation, Groningen.

171. Kees Keizer (2010). The spreading of disorder. ICS-dissertation, Groningen. 
172. Michael Mäs (2010). The diversity puzzle. Explaining clustering and polarization of opinions. ICS-dissertation, Groningen.

173. Marie-Louise Damen (2010). Cultuurdeelname en CKV. Studies naar effecten van kunsteducatie op de cultuurdeelname van leerlingen tijdens en na het voortgezet onderwijs. ICS-dissertation, Utrecht.

174. Marieke van de Rakt (2011). Two generations of crime: The intergenerational transmission of convictions over the life course. ICS-dissertation, Nijmegen.

175. Willem Huijnk (2011). Family life and ethnic attitudes. The role of the family for attitudes towards intermarriage and acculturation among minority and majority groups. ICS-dissertation, Utrecht.

176. Tim Huijts (2011). Social ties and health in Europe. Individual associations, crossnational variations, and contextual explanations. ICS-dissertation, Nijmegen.

177. Wouter Steenbeek (2011). Social and physical disorder. How community, business presence and entrepreneurs influence disorder in Dutch neighborhoods. ICSdissertation, Utrecht.

178. Miranda Vervoort (2011). Living together apart? Ethnic concentration in the neighborhood and ethnic minorities' social contacts and language practices. ICSdissertation, Utrecht.

179. Agnieszka Kanas (2011). The economic performance of immigrants. The role of human and social capital. ICS-dissertation, Utrecht.

180. Lea Ellwardt (2011). Gossip in organizations. A social network study. ICSdissertation, Groningen.

181. Annemarije Oosterwaal (2011). The gap between decision and implementation. Decision making, delegation and compliance in governmental and organizational settings. ICS-dissertation, Utrecht.

182. Natascha Notten (2011). Parents and the media. Causes and consequences of parental media socialization. ICS-dissertation, Nijmegen.

183. Tobias Stark (2011). Integration in schools. A process perspective on students' interethnic attitudes and interpersonal relationships. ICS-dissertation, Groningen.

184. Giedo Jansen (2011). Social cleavages and political choices. Large-scale comparisons of social class, religion and voting behavior in Western democracies. ICSdissertation, Nijmegen.

185. Ruud van der Horst (2011). Network effects on treatment results in a closed forensic psychiatric setting. ICS-dissertation, Groningen.

186. Mark Levels (2011). Abortion laws in European countries between 1960 and 2010. Legislative developments and their consequences for women's reproductive decisionmaking. ICS-dissertation, Nijmegen.

187. Marieke van Londen (2012). Exclusion of ethnic minorities in the Netherlands. The effects of individual and situational characteristics on opposition to ethnic policy and ethnically mixed neighbourhoods. ICS-dissertation, Nijmegen. 
188. Sigrid M. Mohnen (2012). Neighborhood context and health: How neighborhood social capital affects individual health. ICS-dissertation, Utrecht.

189. Asya Zhelyazkova (2012). Compliance under controversy: Analysis of the transposition of European directives and their provisions. ICS-dissertation, Utrecht.

190. Valeska Korff (2012). Between cause and control: Management in a humanitarian organization. ICS-dissertation, Groningen.

191. Maike Gieling (2012). Dealing with diversity: Adolescents' support for civil liberties and immigrant rights. ICS-dissertation, Utrecht.

192. Katya Ivanova (2012). From parents to partners: The impact of family on romantic relationships in adolescence and emerging adulthood. ICS-dissertation, Groningen.

193. Jelmer Schalk (2012). The performance of public corporate actors: Essays on effects of institutional and network embeddedness in supranational, national, and local collaborative contexts. ICS-dissertation, Utrecht.

194. Alona Labun (2012). Social networks and informal power in organizations. ICSdissertation, Groningen.

195. Michał Bojanowski (2012). Essays on social network formation in heterogeneous populations: Models, methods, and empirical analyses. ICS-dissertation, Utrecht.

196. Anca Minescu (2012). Relative group position and intergroup attitudes in Russia. ICS-dissertation, Utrecht.

197. Marieke van Schellen (2012). Marriage and crime over the life course. The criminal careers of convicts and their spouses. ICS-dissertation, Utrecht.

198. Mieke Maliepaard (2012). Religious trends and social integration: Muslim minorities in the Netherlands. ICS-dissertation, Utrecht.

199. Fransje Smits (2012). Turks and Moroccans in the Low Countries around the year 2000: determinants of religiosity, trend in religiosity and determinants of the trend. ICS-dissertation, Nijmegen.

200. Roderick Sluiter (2012). The diffusion of morality policies among Western European countries between 1960 and 2010. A comparison of temporal and spatial diffusion patterns of six morality and eleven non-morality policies. ICS-dissertation, Nijmegen.

201. Nicoletta Balbo (2012). Family, friends and fertility. ICS-dissertation, Groningen.

202. Anke Munniksma (2013). Crossing ethnic boundaries: Parental resistance to and consequences of adolescents' cross-ethnic peer relations. ICS-dissertation, Groningen.

203. Anja Abendroth (2013). Working women in Europe. How the country, workplace, and family context matter. ICS-dissertation, Utrecht.

204. Katia Begall (2013). Occupational hazard? The relationship between working conditions and fertility. ICS-dissertation, Groningen.

205. Hidde Bekhuis (2013). The popularity of domestic cultural products: Cross- national differences and the relation to globalization. ICS-dissertation, Utrecht. 
206. Lieselotte Blommaert (2013). Are Joris and Renske more employable than Rashid and Samira? A study on the prevalence and sources of ethnic discrimination in recruitment in the Netherlands using experimental and survey data. ICS-dissertation, Utrecht.

207. Wiebke Schulz (2013). Careers of men and women in the 19th and 20th centuries. ICS-dissertation, Utrecht.

208. Ozan Aksoy (2013). Essays on social preferences and beliefs in non-embedded social dilemmas. ICS-dissertation, Utrecht.

209. Dominik Morbitzer (2013). Limited farsightedness in network formation. ICSdissertation, Utrecht.

210. Thomas de Vroome (2013). Earning your place: The relation between immigrants' economic and psychological integration in the Netherlands. ICS-dissertation, Utrecht.

211. Marloes de Lange (2013). Causes and consequences of employment flexibility among young people. Recent developments in the Netherlands and Europe. ICSdissertation, Nijmegen.

212. Roza Meuleman (2014). Consuming the nation. Domestic cultural consumption: Its stratification and relation with nationalist attitudes. ICS-dissertation, Utrecht.

213. Esther Havekes (2014). Putting interethnic attitudes in context. The relationship between neighbourhood characteristics, interethnic attitudes and residential behaviour. ICS-dissertation, Utrecht.

214. Zoltán Lippényi (2014). Transitions toward an open society? Intergenerational occupational mobility in Hungary in the 19th and 20th centuries. ICS-dissertation, Utrecht

215. Anouk Smeekes (2014). The presence of the past: Historical rooting of national identity and current group dynamics. ICS-dissertation, Utrecht.

216. Michael Savelkoul (2014). Ethnic diversity and social capital. Testing underlying explanations derived from conflict and contact theories in Europe and the United States. ICS-dissertation, Nijmegen.

217. Martijn Hogerbrugge (2014). Misfortune and family: How negative events, family ties, and lives are linked. ICS-dissertation, Utrecht.

218. Gina Potarca (2014). Modern love. Comparative insights in online dating preferences and assortative mating. ICS-dissertation, Groningen.

219. Mariska van der Horst (2014). Gender, aspirations, and achievements: Relating work and family aspirations to occupational outcomes. ICS-dissertation, Utrecht.

220. Gijs Huitsing (2014). A social network perspective on bullying. ICS dissertation, Groningen.

221. Thomas Kowalewski (2015). Personal growth in organizational contexts. ICSdissertation, Groningen.

222. Manu Muñoz-Herrera (2015). The impact of individual differences on network relations: Social exclusion and inequality in productive exchange and coordination games. ICS-dissertation, Groningen. 
223. Tim Immerzeel (2015). Voting for a change. The democratic lure of populist radical right parties in voting behavior. ICS-dissertation, Utrecht.

224. Fernando Nieto Morales (2015). The control imperative: Studies on reorganization in the public and private sectors. ICS-dissertation, Groningen.

225. Jellie Sierksma (2015). Bounded helping: How morality and intergroup relations shape children's reasoning about helping. ICS-dissertation, Utrecht.

226. Tinka Veldhuis (2015). Captivated by fear. An evaluation of terrorism detention policy. ICS-dissertation, Groningen.

227. Miranda Visser (2015). Loyality in humanity. Turnover among expatriate humanitarian aid workers. ICS-dissertation, Groningen.

228. Sarah Westphal (2015). Are the kids alright? Essays on postdivorce residence arrangements and children's well-being. ICS-dissertation, Utrecht.

229. Britta Rüschoff (2015). Peers in careers: Peer relationships in the transition from school to work. ICS-dissertation, Groningen.

230. Nynke van Miltenburg (2015). Cooperation under peer sanctioning institutions: Collective decisions, noise, and endogenous implementation. ICS-dissertation, Utrecht.

231. Antonie Knigge (2015). Sources of sibling similarity. Status attainment in the Netherlands during modernization. ICS-dissertation, Utrecht.

232. Sanne Smith (2015). Ethnic segregation in friendship networks. Studies of its determinants in English, German, Dutch, and Swedish school classes. ICS- dissertation, Utrecht.

233. Patrick Präg (2015). Social stratification and health. Four essays on social determinants of health and wellbeing. ICS-dissertation, Groningen.

234. Wike Been (2015). European top managers' support for work-life arrangements. ICS- dissertation, Utrecht.

235. André Grow (2016). Status differentiation: New insights from agent-based modeling and social network analysis. ICS-dissertation, Groningen.

236. Jesper Rözer (2016). Family and personal networks. How a partner and children affect social relationships. ICS-dissertation, Utrecht.

237. Kim Pattiselanno (2016). At your own risk: The importance of group dynamics and peer processes in adolescent peer groups for adolescents' involvement in risk behaviors. ICS-dissertation, Groningen.

238. Vincenz Frey (2016). Network formation and trust. ICS-dissertation, Utrecht.

239. Rozemarijn van der Ploeg (2016). Be a buddy, not a bully? Four studies on social and emotional processes related to bullying, defending, and victimization. ICS-dissertation, Groningen.

240. Tali Spiegel (2016). Identity, career trajectories and wellbeing: A closer look at individuals with degenerative eye conditions. ICS-dissertation, Groningen.

241. Felix Tropf (2016). Social Science Genetics and Fertility. ICS-dissertation, Groningen. 
242. Sara Geven (2016). Adolescents problem behavior in school: the role of peer networks. ICS-dissertation, Utrecht.

243. Josja Rokven (2016). The victimization-offending relationship from a longitudinal perspective. ICS-dissertation, Nijmegen.

244. Maja Djundeva (2016). Healthy ageing in context: Family welfare state and the life course. ICS-dissertation, Groningen.

245. Mark Visser (2017). Inequality between older workers and older couples in the Netherlands. A dynamic life course perspective on educational and social class differences in the late career. ICS-dissertation, Nijmegen.

246. Beau Oldenburg (2017). Bullying in schools: The role of teachers and classmates. ICS-dissertation, Groningen.

247. Tatang Muttaqin (2017). The education divide in Indonesia: Four essays on determinants of unequal access to and quality of education. ICS-dissertation, Groningen.

248. Margriet van Hek (2017). Gender inequality in educational attainment and reading performance. A contextual approach. ICS-dissertation, Nijmgen.

249. Melissa Verhoef (2017). Work schedules, childcare and well-being. Essays on the associations between modern-day job characteristics, childcare arrangements and the well-being of parents and children. ICS-dissertation, Utrecht.

250. Timo Septer (2017). Goal priorities, cognition and conflict: Analyses of cognitive maps concerning organizational changes. ICS-dissertation, Groningen.

251. Bas Hofstra (2017). Online Social Networks: Essays on Membership, Privacy, and Structure. ICS-dissertation, Utrecht.

252. Yassine Khoudja (2018). Women's labor market participation across ethnic groups: The role of household conditions, gender role attitudes, and religiosity in different national context ICS-dissertation, Utrecht

253. Joran Laméris (2018), Living together in diversity. Whether, why and where ethnic diversity affects social cohesion ICS-dissertation, Nijmegen

254. Maaike van der Vleuten (2018). Gendered Choices. Fields of study of adolescents in the Netherlands ICS-dissertation, Utrecht

255. Mala Sondang Silitonga (2018). Corruption in Indonesia: The impact of institutional change, norms, and networks ICS-dissertation, Groningen

256. Manja Coopmans (2018). Rituals of the past in the context of the present. The role of Remembrance Day and Liberation Day in Dutch society ICS-dissertation, Utrecht

257. Paul Hindriks (2018). The Struggle for Power: Attitudes towards the political participation of ethnic minorities ICS-dissertation, Utrecht

258. Nynke Niezink (2018). Modeling the dynamics of networks and continuous behavior ICS-dissertation, Groningen

259. Simon de Bruijn (2018). Reaching agreement after divorce and separation. Essays on the effectiveness of parenting plans and divorce mediation ICS-dissertation, Utrecht 
260. Susanne van 't Hoff-de Goede (2018). While you were locked up. An empirical study on the characteristics, social surroundings and wellbeing of partners of prisoners in The Netherlands ICS-dissertation, Utrecht

261. Loes van Rijsewijk (2018). Antecedents and Consequences of Helping among Adolescents ICS-dissertation, Groningen

262. Mariola Gremmen (2018). Social network processes and academic functioning. The role of peers in students' school well-being, academic engagement, and academic achievement ICS-dissertation, Groningen

263. Jeanette Renema (2018). Immigrants' support for welfare spending. The causes and consequences of welfare usage and welfare knowledgeability ICS-dissertation, Nijmegen

264. Suwatin Miharti (2018). Community health centers in Indonesia in the era of decentralization. The impact of structure, staff composition and management on health outcomes ICS-dissertation, Groningen

265. Chaïm la Roi (2019). Stigma and stress: Studies on attitudes towards sexual minority orientations and the association between sexual orientation and mental health ICS-dissertation, Groningen

266. Jelle Lössbroek (2019), Turning grey into gold. Employer-employee interplay in an ageing workforce ICS-dissertation, Utrecht

267. Nikki van Gerwen (2019). Employee cooperation through training. A multi-method approach ICS-dissertation, Utrecht

268. Paula Thijs (2019). Trends in cultural conservatism: The role of educational expansion, secularisation, and changing national contexts ICS-dissertation, Nijmegen

269. Renske Verweij (2019). Understanding childlessness: Unravelling the link with genes and the socio-environment ICS-dissertation, Groningen

270. Niels Blom (2019). Partner relationship quality under pressing work conditions. Longitudinal and cross-national investigation ICS-dissertation, Nijmegen

271. Müge Simsek (2019). The dynamics of religion among native and immigrant youth in Western Europe ICS-dissertation, Utrecht

272. Leonie van Breeschoten (2019). Combining a career and childcare: The use and usefulness of work-family policies in European organizations ICS-dissertation, Utrecht

273. Roos van der Zwan (2019). The political representation of ethnic minorities and their vote choice ICS-dissertation, Nijmegen

274. Ashwin Rambaran (2019). The classroom as context for bullying: A social network approach ICS-dissertation, Groningen

275. Dieko Bakker (2019). Cooperation and social control: Effects of preferences, institutions, and social structure ICS-dissertation, Groningen

276. Femke van der Werf (2019). Shadow of a rainbow? National and ethnic belonging in Mauritius ICS-dissertation, Utrecht 
277. Robert Krause (2019). Multiple Imputation for Missing Network Data ICS-dissertation, Groningen

278. Take Sipma (2020). Economic insecurity and populist radical right voting ICSdissertation, Nijmegen

279. Mathijs Kros (2020). The nature of negative contact: studies on interethnic relations in western societies ICS-dissertation, Utrecht

280. Lonneke van den Berg (2020). Time to leave: Individual and contextual explanations for the timing of leaving home ICS-dissertation, Amsterdam

281. Marianne Hooijsma (2020). Clashrooms: Interethnic peer relationships in schools ICS-dissertation, Groningen

282. Marina Tulin (2020). Blind Spots in Social Resource Theory: Essays on the Creation, Maintenance and Returns of Social Capital ICS-dissertation, Amsterdam

283. Tessa Kaufman (2020). Toward Tailored Interventions: Explaining, Assessing, and Preventing Persistent Victimization of Bullying" ICS-dissertation, Groningen

284. Lex Thijssen (2020), Racial and Ethnic Discrimination in Western Labor Markets: Empirical Evidence from Field Experiments ICS-dissertation, Utrecht

285. Lukas Norbutas (2020). Trust on the Dark Web: An analysis of illegal online drug markets ICS-dissertation, Utrecht

286. Tomáš Diviák (2020). Criminal Networks: Actors, Mechanisms, and Structures ICS-dissertation, Groningen

287. Tery Setiawan (2020). VSupport for Interreligious Conflict in Indonesia ICSdissertation, Nijmegen

288. Vera de Bel (2020). The ripple effect in family networks. Relational structures and well-being in divorced and non-divorced families ICS-dissertation, Groningen

289. Diego Palacios (2020). How Context and the Perception of Peers' Behaviors Shape Relationships in Adolescence: A Multiplex Social Network Perspective ICSdissertation, Groningen

290. Saskia Glas (2020). Where are the Muslim Feminists?: Religiosity and Support for Gender Equality in the Arab region ICS-dissertation, Nijmegen

291. Tomas Turner-Zwinkels (2020). A New Macro-Micro Approach to the Study of Political Careers: Theoretical, Methodological and Empirical Challenges and Solutions ICS-dissertation, Groningen

292. Lotte Scheeren (2020). Not on the Same Track? Tracking Age and Gender Inequality in Education ICS-dissertation, Amsterdam

293. Joris Broere (2020). Essays on how social network structure affects asymmetric coordination and trust ICS-dissertation, Utrecht 
Network structure has been put forward as a possible solution to cooperation problems and social dilemmas. In the first part of this dissertation, the effect of network structure is studied for a socia dilemma situation involving asymmet ic coordination. The effects of network properties on these coordination problems are studied by means of agent-based models, models of statistical physics, and a laboratory experiment. In the second part, we study the question on how general trust can be established in societies that are becoming more individualistic. The theory known as organic solidarity argues that division of labor is one of the key foundations of trust and solidarity between people. This proposition is studied by quantifying aspects of the division of labor. We show that these aspects are strongly related to general trust. 